\title{
Biologically Plausible Models of Neurite Outgrowth
}

\section{Gregor Alexander Campbell Kiddie}

A thesis submitted in partial fulfilment of the

\author{
requirements for the
}

degree of Doctor of Philosophy

Computing Science and Mathematics

School of Natural Sciences

University of Stirling

Stirling

Scotland

October 31, 2011 


\section{CONTENTS}

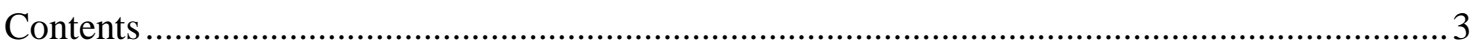

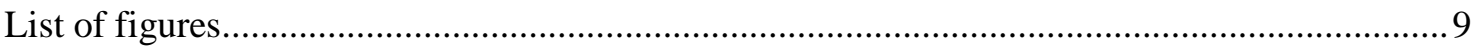

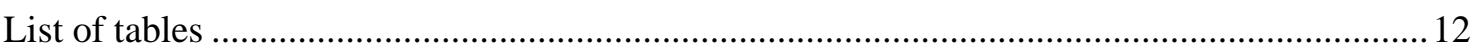

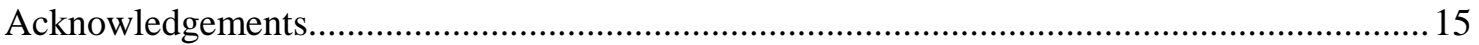

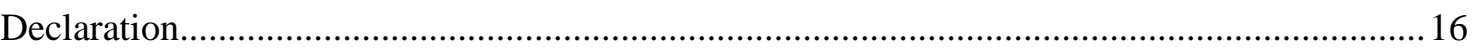

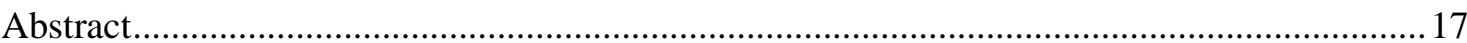

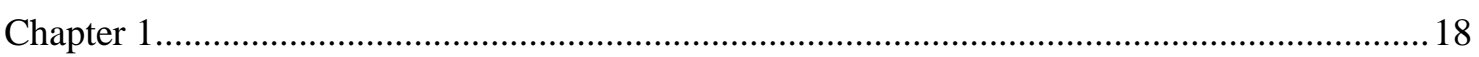

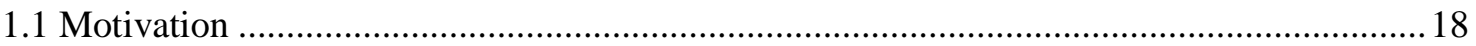

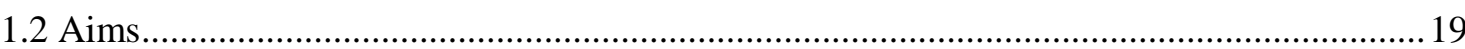

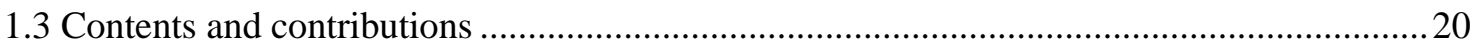

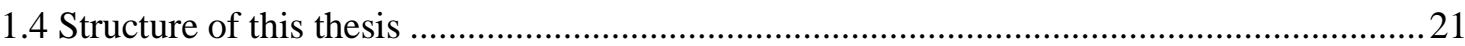

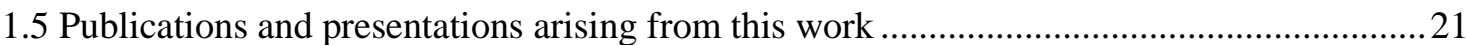

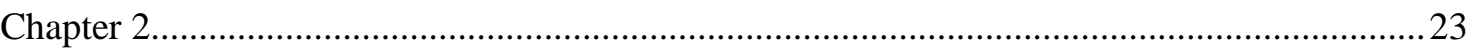

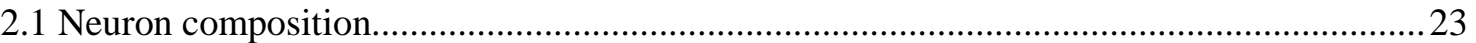

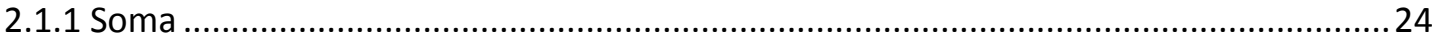

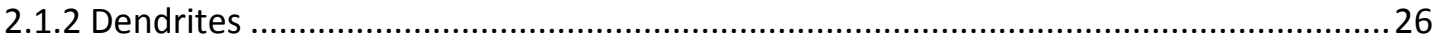

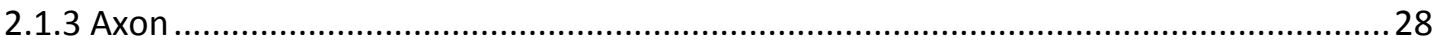

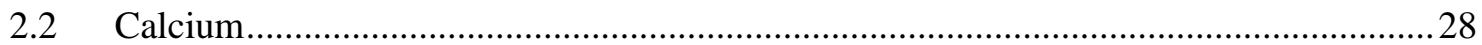

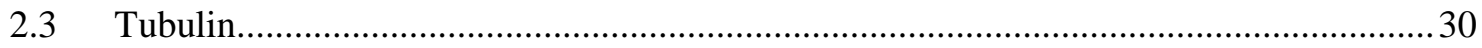




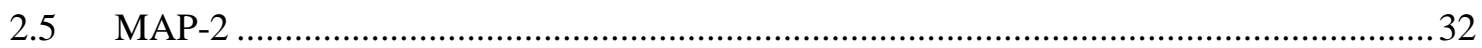

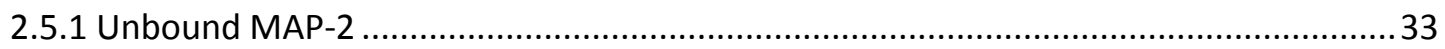

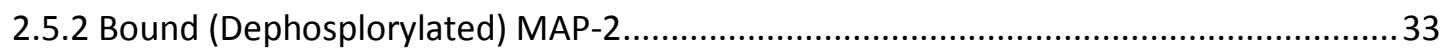

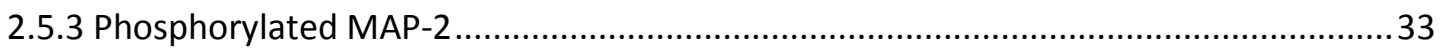

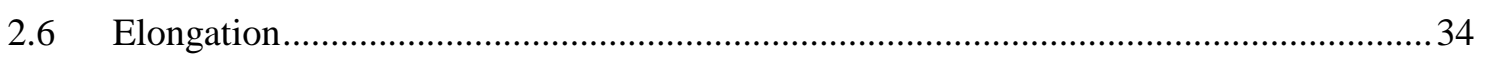

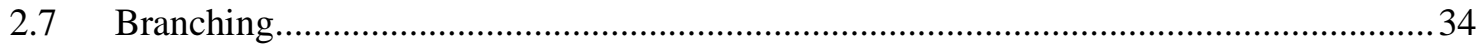

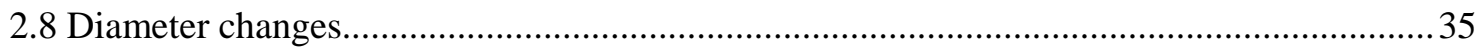

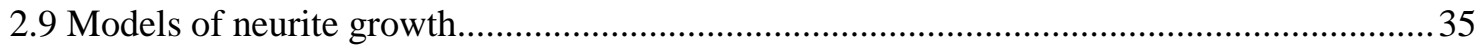

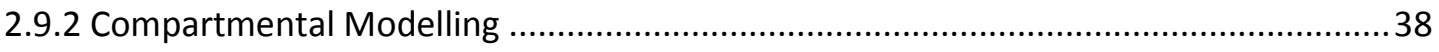

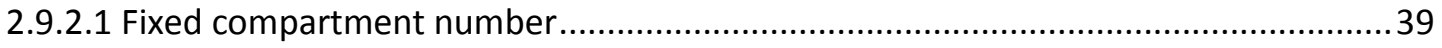

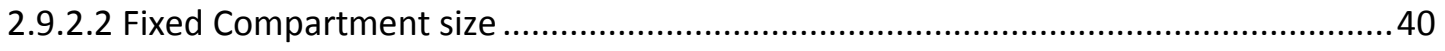

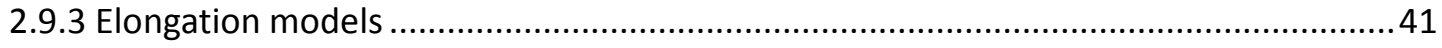

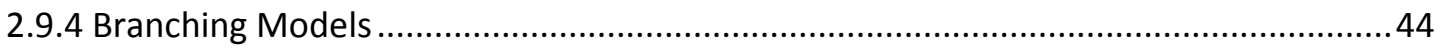

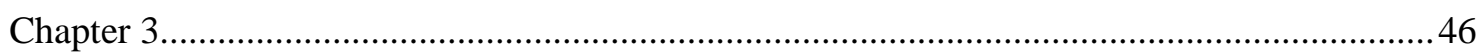

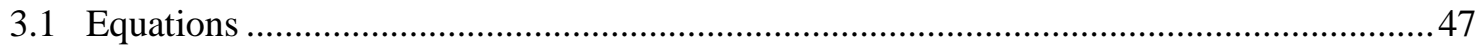

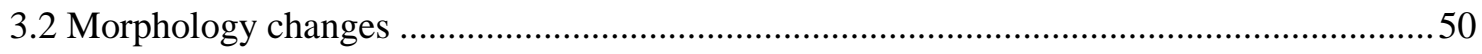

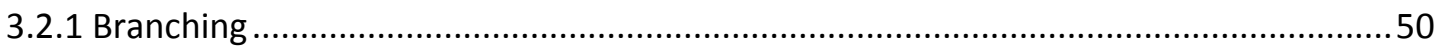

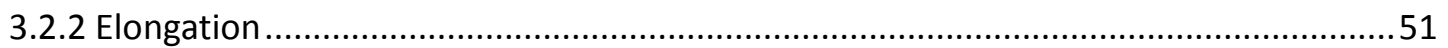

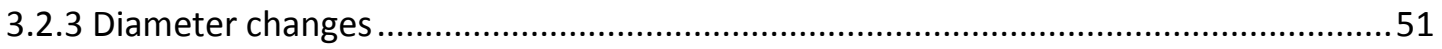

3.3 Elements of biological background not considered by the model ...................................... 51

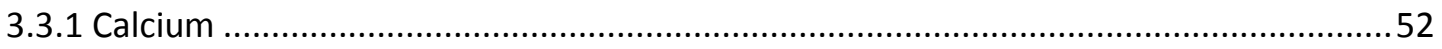

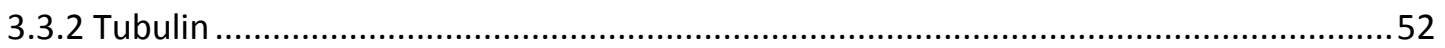

3.3.3 Elongation ....................................................................................... 52

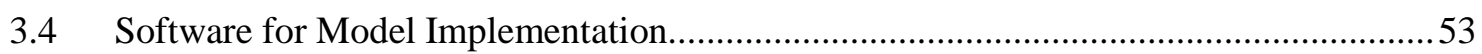




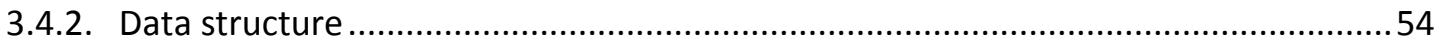

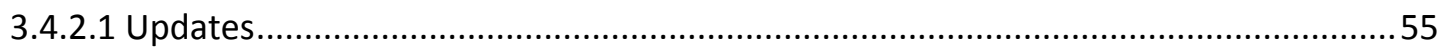

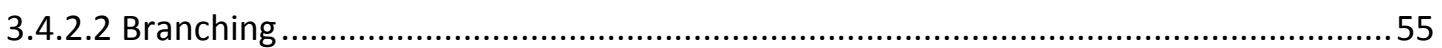

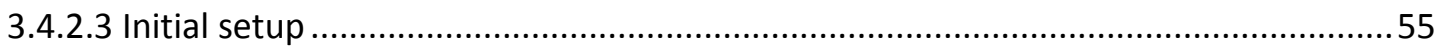

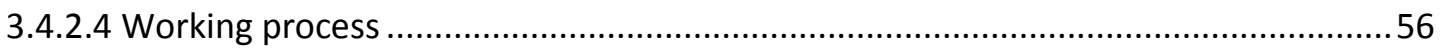

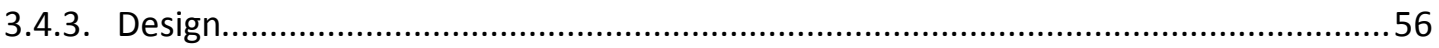

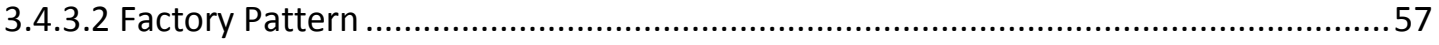

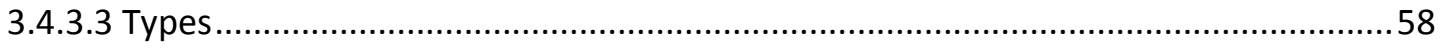

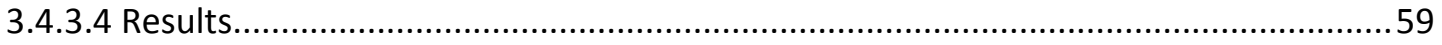

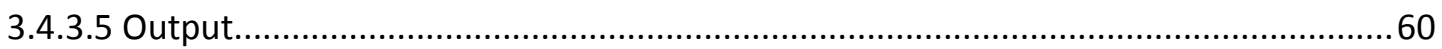

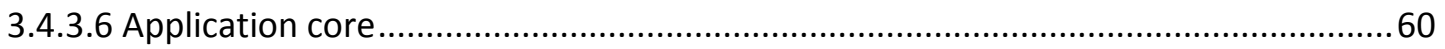

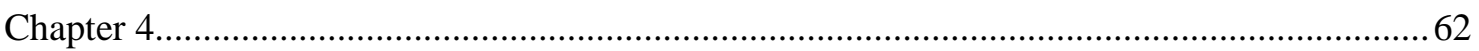

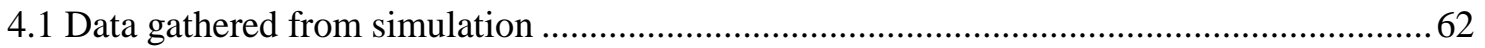

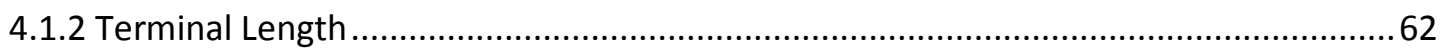

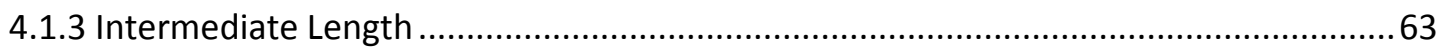

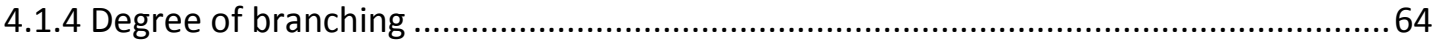

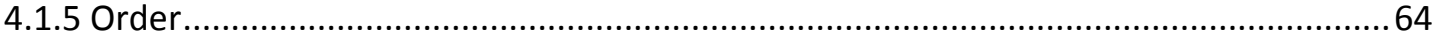

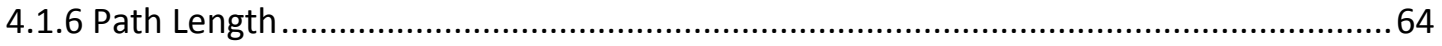

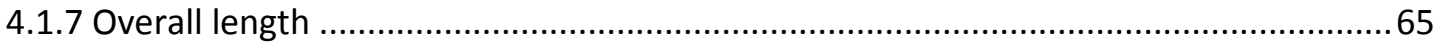

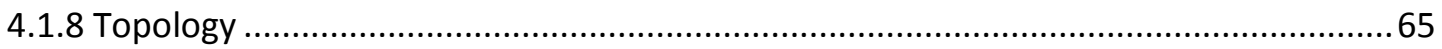

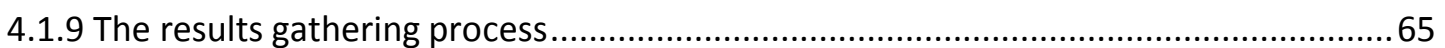

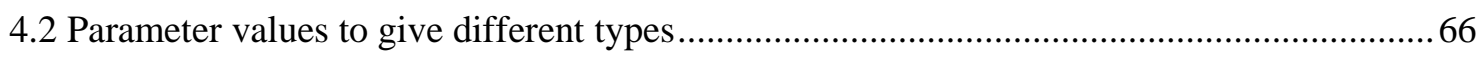

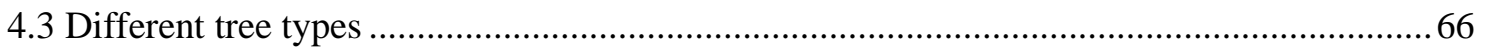

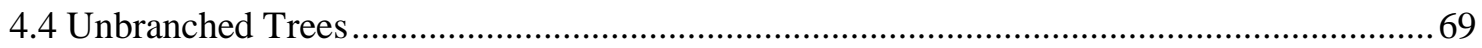

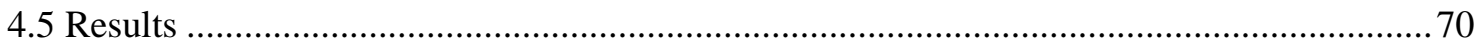

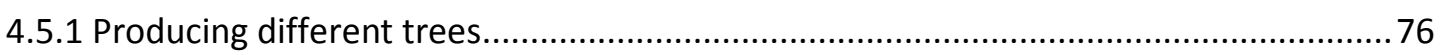

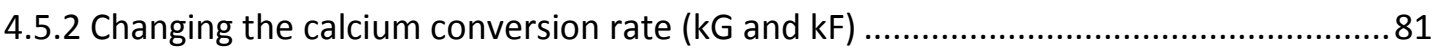

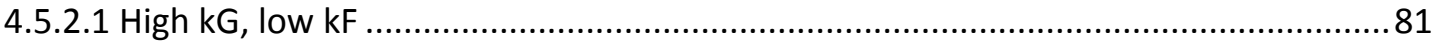

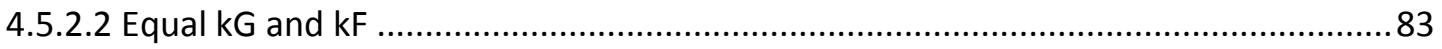


4.5.2.3 Low kG, high kF

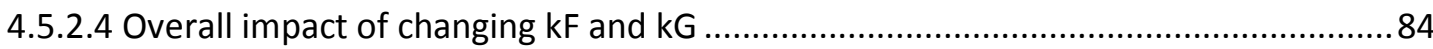

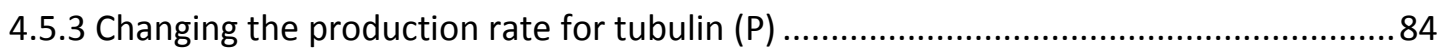

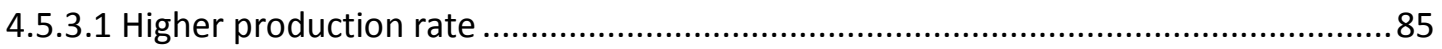

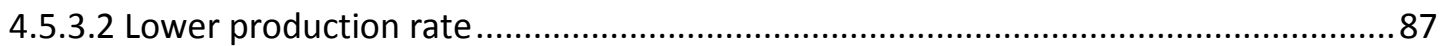

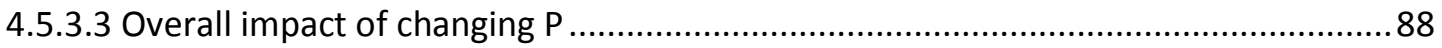

4.5.4 Changing the production rate for unbound MAP-2 (S) ................................................ 89

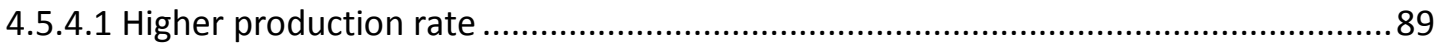

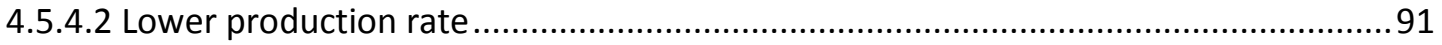

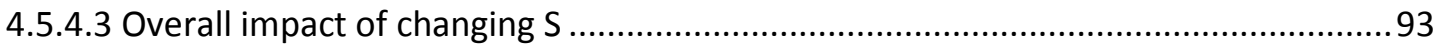

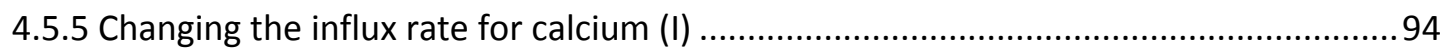

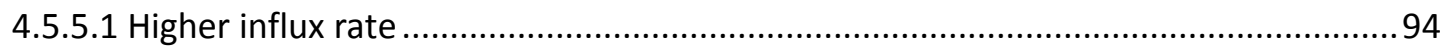

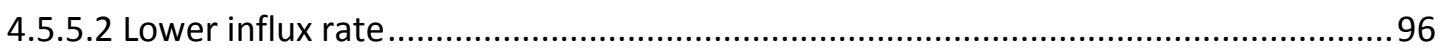

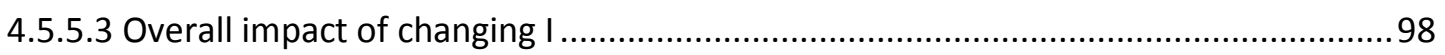

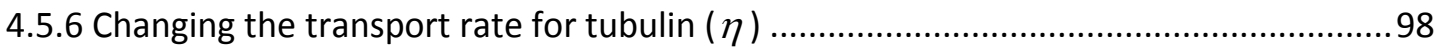

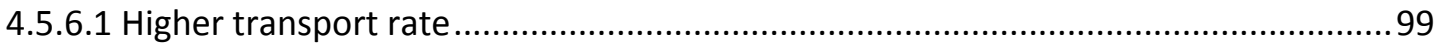

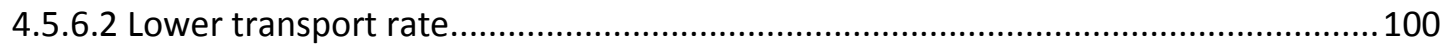

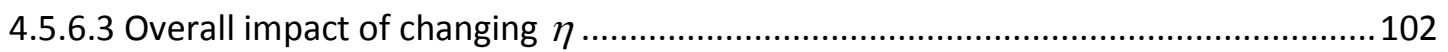

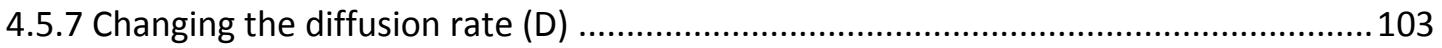

4.5.7.1 Higher diffusion rate ............................................................................................. 103

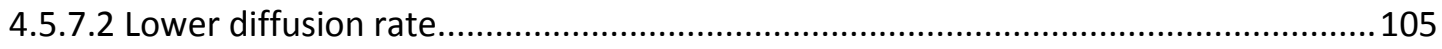

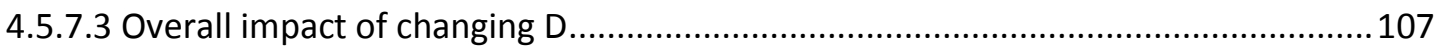

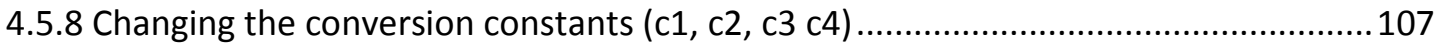

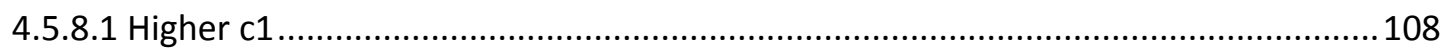

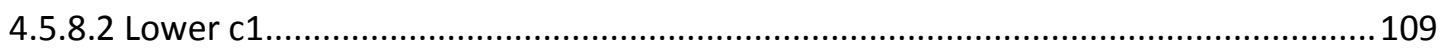

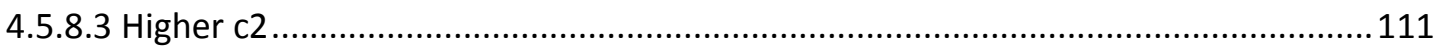

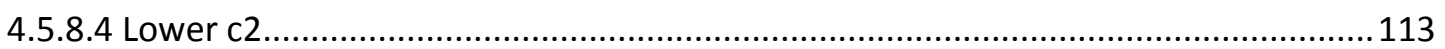

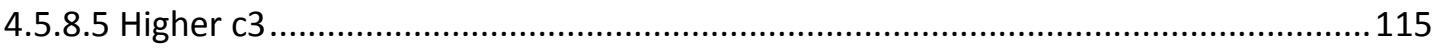

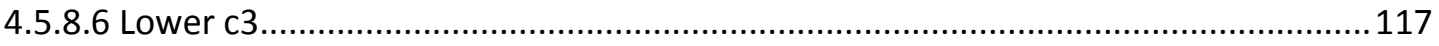

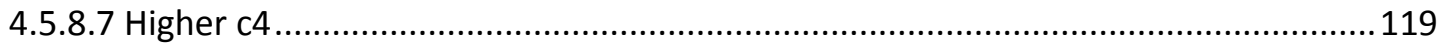

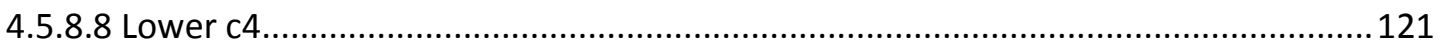

4.5.8.9 Overall impact of changing the $\mathrm{c}$ constants .........................................................123

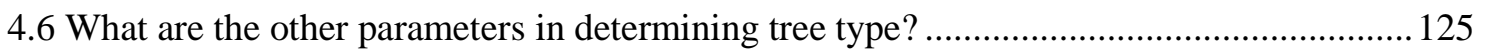




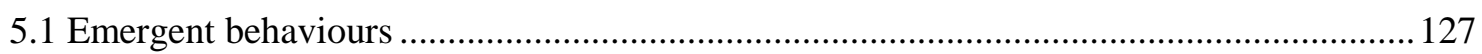

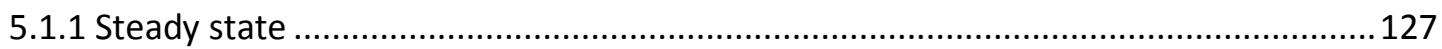

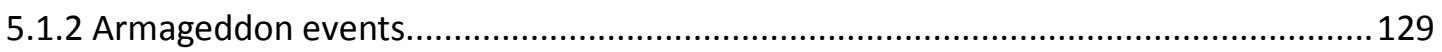

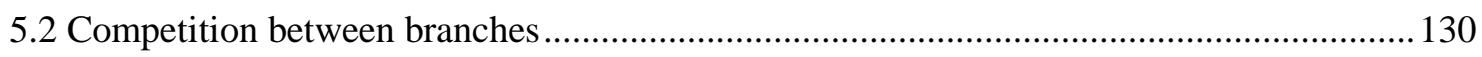

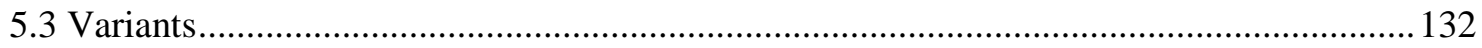

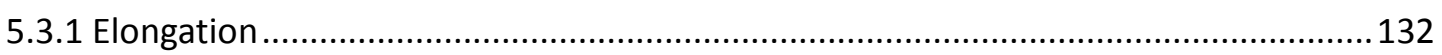

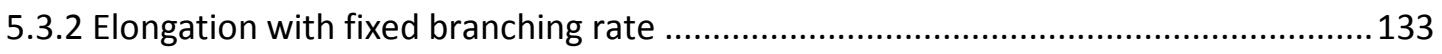

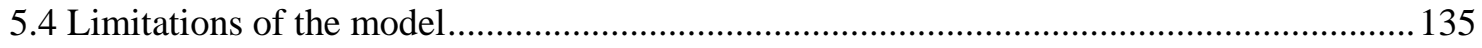

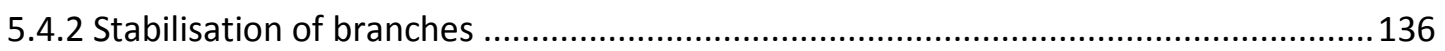

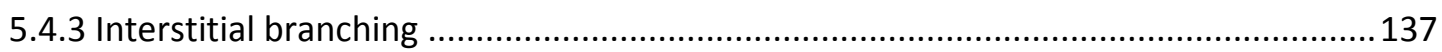

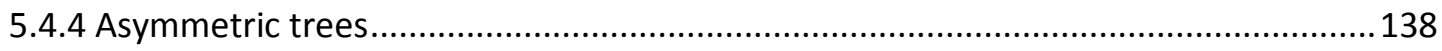

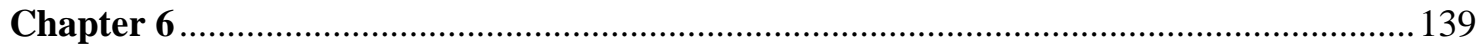

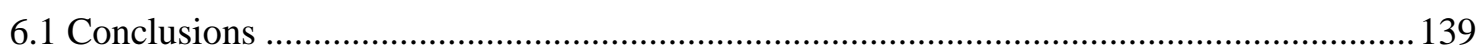

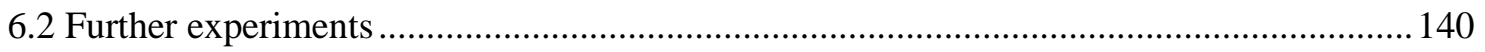

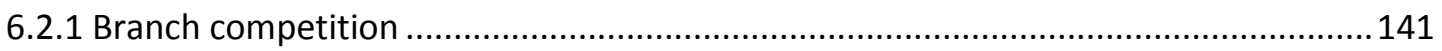

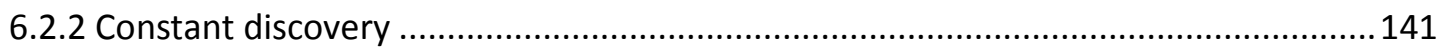

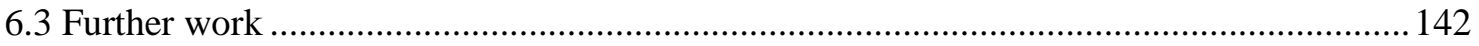

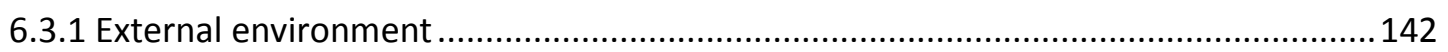

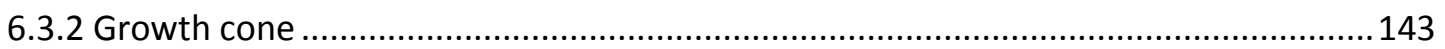

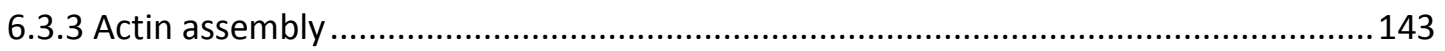

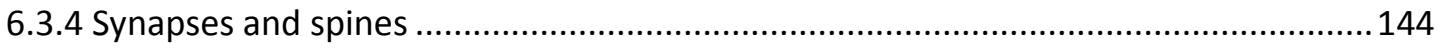

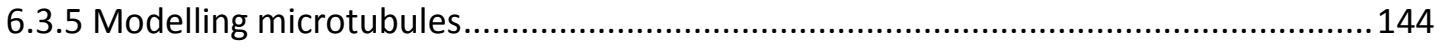

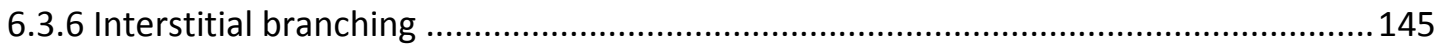

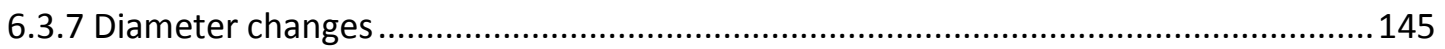

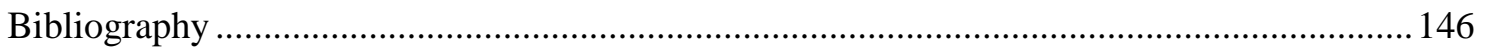


Image References and attribution 


\section{LIST OF FIGURES}

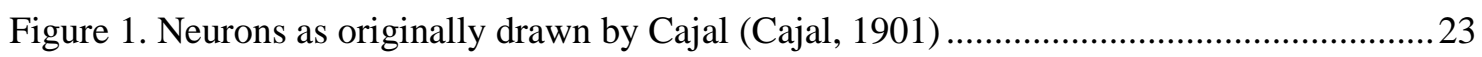

Figure 2. Cell structure (Vanderblit University medical centre) …........................................... 24

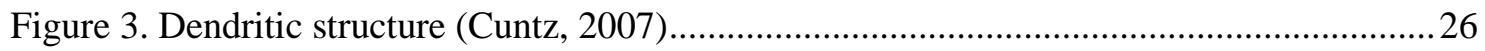

Figure 4. Structure of microtubules(Baulin, 2005) ................................................................... 31

Figure 5. MAP-2 states as regulated by the rate change constants (c1 - c4) and the rate change modulators $\mathrm{F}$ and $\mathrm{G}$ which are functions of calcium that quantify how the calcium concentration affects the phosphorylation and dephosphorylation rates.................................... 34

Figure 6. Discretization of Elongation Model. (Top) early time. (Bottom) later time. ...............40

Figure 7. Fixed Size Compartment Model...........................................................................

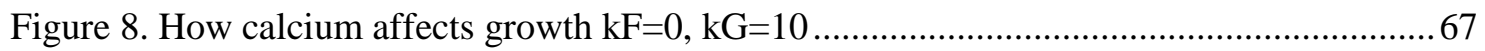

Figure 9. Switching between phase states (LEFT) high c3/c4 ratio, (right) low c3/c4 ratio......68

Figure 10. Calcium in the initial terminal segment over time. .................................................... 71

Figure 11. MAP-2 states in relation to each other in the initial terminal segment over time..... 72

Figure 12. Free tubulin in the initial terminal segment over time. ........................................... 73

Figure 13. (Left) The probability of branching in the initial terminal segment over time. (Right) The amount of elongation in the initial terminal segment over time. .73

Figure 14. (TOP left) the histogram for terminal length, (TOP RIGHT) the histogram for intermediate length, (BOTTOM LEFT) the histogram for path lengths, (BOTTOM RIGHT) The histogram for the order.

Figure 15. (left) Similar length segments, (centre) Shorter terminal segments, (right) Longer terminal segments (the default model).

Figure 16. (TOP left)Terminal, (TOP right)Intermediate Lengths, (BOTTOM LEFT) PATH

LENGTH, (BOTTOM RIGHT) Order For similar length tree. . .77

Figure 17. (TOP left) Terminal (TOP right) Intermediate (BOTTOM LEFT) PATH lengths and (BOTTOM RIGHT) ORDER for short tree .78 
Figure 18. (TOP left)Terminal (TOP right)Intermediate (BOTTOM LEFT) PATH Lengths and (bottom right) order For Higher kG .82

Figure 19. (TOP left)Terminal (TOP right)Intermediate (bottom left) path Lengths and (bottom right) Order For higher tubulin production. .86

Figure 20. (Top left)Terminal (top right)Intermediate (bottom left) path Lengths and (bottom right) order For lower tubulin production

Figure 21. Overall impact of changing tubulin production .88

Figure 22. (top left)Terminal (top right)Intermediate (bottom left) path Lengths and (bottom right) order For Higher unbound map-2 production. .90

Figure 23. (left)Terminal (right)Intermediate Lengths For lower unbound map-2 production..92 Figure 24. overall impact of changing unbound map-2 production.

Figure 25. (top left) Terminal (top right) Intermediate (bottom left) path Lengths and (bottom right) order For higher calcium influx .95

Figure 26. (top left) Terminal (top right) Intermediate (bottom left) Path Lengths and (bottom right) order For lower calcium influx .96

Figure 27. overall impact of changing calcium influx .98

Figure 28. (top left) Terminal (top right) Intermediate (bottom left) path Lengths and (bottom right) order For higher active transport

Figure 29. (TOP left)Terminal (TOP right)Intermediate (bottom left) path Lengths and (bottom right) order For lower active transport rate. 101

Figure 30. Overall impact of changing active transport rate 102

Figure 31. (top left)Terminal (top right)Intermediate (bottom left) path Lengths and (bottom right) order For higher diffusion rate. 104

Figure 32. (top left) Terminal (top right) Intermediate (bottom left) path Lengths and (bottom right) order For lower diffusion rate... 105

Figure 33. overall impact of changing the diffusion constant 107

Figure 34. (top left) Terminal (top right) Intermediate (bottom left) path Lengths and (bottom right) order For higher c1 108 
Figure 35. (top left) Terminal (top right) Intermediate (bottom left) path Lengths and (bottom right) order For lower c1 110

Figure 36. (top left) Terminal (top right) Intermediate (bottom right) path Lengths and (bottom right) order For Higher c2

Figure 37. (top left) Terminal (top right) Intermediate (bottom left) path Lengths and (order)

For lower c2.

Figure 38. (top left) Terminal (top right) Intermediate (bottom left) path Lengths and (bottom right) order For higher c3 116

Figure 39. (top left) Terminal (top right) Intermediate (bottom left) path Lengths and (bottom right) order For lower c3.

Figure 40. (top left) Terminal (top right) Intermediate (bottom left) path Lengths and (bottom right) order For higher c4 120

Figure 41. (top left) Terminal (top right) Intermediate (bottom left) path Lengths and (bottom right) order For lower c4.

Figure 42. overall impact of changing $\mathrm{c} 1$ 123

Figure 43. overall impact of changing c2

Figure 44. overall impact of changing c3 124

Figure 45. overall impact of changing c4 124

Figure 46. Steady state system. 127

Figure 47. oscillations around a point in three consecutive time bins 128

Figure 48. retraction in unstable branch 128

Figure 49. a tubulin starved branch and well fed branch. 130

Figure 50. (left) little tubulin, (center) steady state, (right) constant elongation..... 132

Figure 51. Elongation with fixed branching 134 


\section{LIST OF TABLES}

Table 1. Model Classification by Type

Table 2. Model Classification by Mathematical Techniques. 38

Table 3. The parameter set for producing trees with long terminals. .71

Table 4. Rat basal pyramidal cell dendrite data taken from Larkman(1991), compared to the data resulting from the simulation of the model.

Table 4. Statistical data for the Similar length tree. .78

Table 5. Statistical data for short terminal tree. 79

Table 6. Rate constants for the three types of neuronal development. .80

Table 7. The values for a high kG.

Table 8. Statistical data for the high kG tree.

Table 9. The values for equal $\mathrm{kF}$ and $\mathrm{kG}$.

Table 10. The values for high $\mathrm{kF}$ and low $\mathrm{kG}$

Table 11. The higher production rate of tubulin. .85

Table 12. Statistical data for higher tubulin tree. .86

Table 13. The lower production rate for tubulin. .87

Table 14. Statistical data for lower tubulin tree. .88

Table 15. The higher production rate for unbound MAP-2. .89

Table 16. Statistical data for higher unbound MAP-2 tree. 91 
Table 17. The lower production rate for unbound MAP-2.

Table 18. Statistical data for short terminal tree

Table 19. The higher influx rate for calcium .94

Table 20. Statistical data for higher calcium influx tree. .96

Table 21. The lower influx rate for calcium. .96

Table 22. Statistical data for lower calcium influx tree. .97

Table 23. The higher rate for the active transport rate. .99

Table 24. Statistical data for higher active transport tree. 100

Table 25. The lower rate for the active transport rate 100

Table 26. Statistical data for the lower active transport tree. 102

Table 27. The higher diffusion rate. .103

Table 28. Statistical data for higher diffusion tree... 105

Table 29. The lower diffusion rate. 105

Table 30. Statistical data for lower diffusion tree. 106

Table 31. The higher c1. 108

Table 32. Statistical data for lower c1 tree. 109

Table 33. The lower c1. 109

Table 34. Statistical data for lower c1 tree.

Table 35. The higher c2. 
Table 36. Statistical data for higher c2 tree.

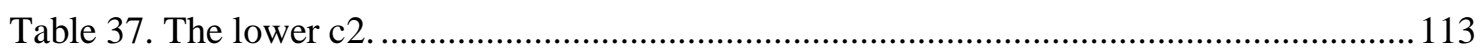

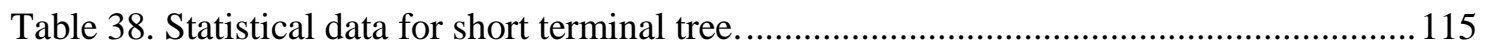

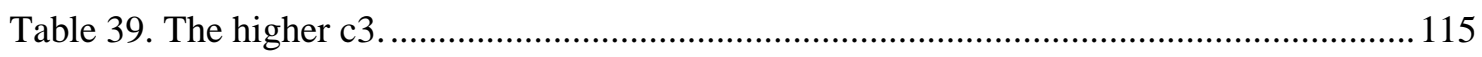

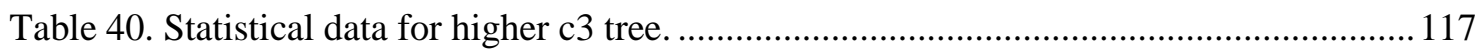

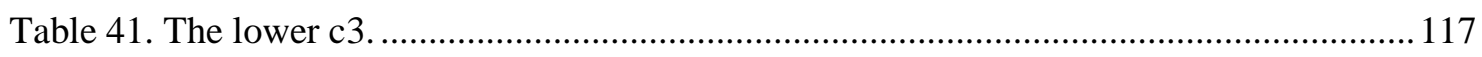

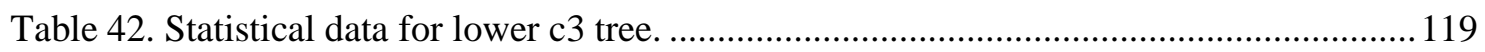

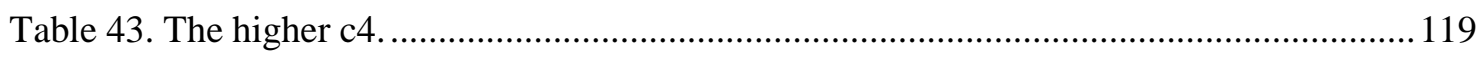

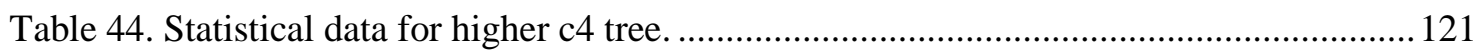

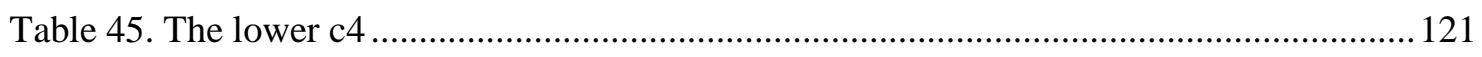

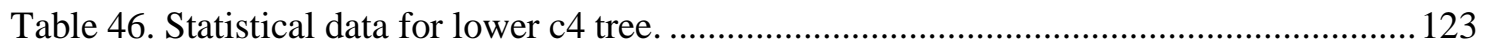




\section{ACKNOWLEDGEMENTS}

I would like to acknowledge the support from my supervisor Bruce Graham. My thanks go to my wife Mary, whose infinite support, understanding, and encouragement has maintained this project. I am also grateful to Stuart and Jenna for providing me with the competitive edge I obviously require, and to the Hussars for providing the drinks. Further thanks go to Arjen van Ooyen for several useful discussions, and the EPSRC (Grant GR/R89769/01) for funding this work. 


\section{DECLARATION}

I certify that this dissertation reports original work by me, except where otherwise stated. This dissertation has not previously been accepted in substance for any degree and is not concurrently submitted in candidature for any degree other than Doctor of Philosophy of the University of Stirling.

Signature:

Date: 


\section{ABSTRACT}

The growth of a neuronal dendritic tree depends on the neuron's internal state and the environment within which it is situated. Different types of neuron develop dendritic trees with specific characteristics, such as the average number of terminal branches and the average length of terminal and intermediate segments. A key aspect of the growth process is the construction of the microtubule cytoskeleton within the dendritic tree. Neurite elongation requires assembly of microtubules from free tubulin at the growth cone. The stability of microtubule bundles is an important factor in determining how likely it is for a growth cone to split to form new daughter branches. Microtubule assembly rates and bundle stability are controlled by microtubuleassociated proteins, principally MAP2 in dendrites. Extending previous work (Hely et al, J. Theor. Biol. 210:375-384, 2001) I have developed a mathematical model of neurite outgrowth in which elongation and branching rates are determined by the phosphorylation state of MAP2 at the tips of each terminal branch. Tubulin and MAP2 are produced in the cell body and transported along the neurite by a combination of diffusion and active transport. Microtubule (dis)assembly at neurite tips is a function of tubulin concentration. The rate of assembly depends on the amount of unphosphorylated MAP2 bound to the microtubules and linking them together. Phosphorylation of MAP2 destroys its linking capability and destabilises the microtubule bundles. Each terminal has a probability of branching that depends on the phosphorylation of MAP2 which, in turn, is a function of calcium concentration. Results from this model show that changes in the (de)phosphorylation rates of MAP2 affect the topology of the final dendritic tree. Higher phosphorylation promotes branching and results in trees with many short terminal branches and relatively long intermediate segments. Reducing phosphorylation promotes elongation and inhibits branching. 


\section{CHAPTER 1}

\section{Introduction}

Research into how neurological structures grow is essential to a wide range of medical fields. The parts of the neuron explored in this thesis are the dendrites, the tree-like structures which grow from the cell body. Experimental observations of the internal nature of neurons are limited due to the fragility of the cells in question, researchers cannot insert probes to measure the amounts of chemicals in a cell without killing it. This leaves researchers with only those elements which can be measured without interacting with the cell, such as its shape, lengths, diameters, and complexity. This is referred to as the morphology of the cell. Those researchers looking to regrow damaged neurons or grow artificial ones are dependent on having an accurate and plausible model with which to compare their own work. Research into the development of neurons is also in the minority with the majority of researchers working with fully developed neurons.

\subsection{Motivation}

The majority of models of neuronal development have concentrated on statistical models which ignore the true underlying chemical mechanisms(Berry, 1975; Carriquiry et al, 1991; Li et al, 1991; Miller and Samuels, 1996; Nowakowski et al, 1991; van Pelt and Uylings, 1999; van Pelt et al, 1997; van Pelt el at, 2003; van Veen and van Pelt, 1992). Understanding which biological mechanisms take place during growth has been studied experimentally but few models have been created, and those that have are extremely simple ( $\mathrm{Li}$ et al, 1993; Li et al 1994; Graham and van Ooyen, 2001; Graham and van Ooyen, 2004; Hely et al, 2001; McLean et al, 2003; van Ooyen etl al, 2001). No amount of statistical models will give the security of an accurate process for researchers looking to provide context for their own work. Models which are biologically plausible with respect to chemical interactions which have been proven to have an 
effect on the morphology of the cells can be created. These can then be used to further the understanding of the interaction within the cells without needing to disrupt the cells. This would provide a better understanding of the processes and mechanisms involved in the development of the dendritic structure and would provide a solid foundation for further work to expand upon. Researchers working on other fields would have greater confidence that the models they were using based on biological principles were accurate and could be explained mathematically which is not the case with statistical models. Other computational models have taken different approaches, such as branching based on tension within a growth cone due to forces acting on the filopodia (Li et al 1992, 1995; van Veen and van Pelt 1992), and elongation based on a constant lengthening applied during each time step (McLean et al, 2004).

\subsection{Aims}

The aim of this thesis is to create a model in which elongation and branching are generated by a linked process based on biologically plausible principles. This model will be based around the effects of tubulin and its associated microtubule formations. These formations are strongly linked to the microtubule associated protein, MAP-2, in its three states, unbound, bound to microtubules but not phosphorylated, and bound but phosphorylated. The availability or unavailability of resources, such as tubulin or MAP-2 will affect the growth of the tree. The scarcity of resources will be a function of growth as well as diameter changes in branches. Diameter changes are introduced to the model so that during branching events the internal structure widens as its grows in order to support the branches. The model will be consistent with previous models and the statistical models which are used as reference points.

The model will be able to generate a wide variety of trees as detailed by previous experimental work, and by altering the parameters of the model, such as the rate of conversion of MAP-2 between its three states, or the rate at which tubulin is transported through the tree to the tips of the terminals, it will be shown that different types of morphological dendritic structures can be 
produced. This model will then be in a position to be extended by future work to include a wider variety of external interactions.

\subsection{Contents and contributions}

This thesis contains a mathematical model containing formulae detailing the interacting processes and mechanisms for a variety of chemicals observed to have an effect on the development on the dendritic tree of a neuron. It contains an explanation of how these processes and mechanisms interact and the effect they have on producing the morphology of the dendritic structure in understandable and repeatable ways. It discusses ways in which the parameters of the model may be altered in order to produce different results similar to those experimentally discovered and which statistical models have been based upon.

This thesis makes the following contributions.

- Inclusion of further chemical processes and mechanisms into previously explored biologically plausible models.

- Confirmation of an accurate mathematical technique on which to base the model.

- Evidence that more complicated models can match the accuracy of statistical models when modelling morphology.

- Examples of how biologically plausible models can be altered to produce different tree types.

- Suggestions of ways to further extend the work of biologically plausible models in order to move closer to a model which mimics a cell perfectly during its development process. 


\subsection{Structure of this thesis}

This thesis is broken up into six chapters. Chapter one is this introduction. Chapter two details all the chemicals which are included in the model and the ways in which they interact to produce the biological processes which drive the development of the dendritic structure along with different types of models including initiation, elongation, and branching types of models which use a variety of mathematical modelling techniques including ordinary and partial differential equations. Chapter three outlines the formulae which are used in the model which forms the basis of this thesis and describes the software used to generate the data sets based on the mathematical model. Chapter four explains the different tree types we are looking to create, shows the results of the data sets and how by altering the parameters of the model alternative types of trees can be produced. Chapter five shows some interesting behaviours which are not directly related to the generation of the morphology but which have relevance to the biological processes which are being mimicked. It also shows the results of some variants of the model which alter the behaviour of the model in certain ways. Chapter six contains the conclusions and suggests ways in which this model could be extended in the future.

\subsection{Publications and presentations arising from this work}

This work has resulted in the following papers and presentations.

- Kiddie, G., McLean, D., van Ooyen, A. and Graham, B. (2005). Biologically plausible models of neurite outgrowth. In van Pelt, J., Kamermans, M., Levelt, C., van Ooyen, A., Ramakers, G., and Roelfsema, P., editors, Development, dynamics and pathology of neuronal networks: from molecules to functional circuits, Progress in Brain Research, 147, pages 67-80. Elsevier.

- Kiddie, G., van Ooyen, A., and Graham, B. (2005b). A biophysically-based compartmental model of dendritic outgrowth and branching. WiR Workshop on Datadriven Modelling and Computation in Neuroscience. Hohenwart Forum, Germany. 
- Kiddie, G., van Ooyen, A., and Graham, B. (2005c). Modelling the variation in dendritic outgrowth between different neuronal types. Computational Neuroscience Conference (CNS*2005). Madison, Wisconsin, USA.

- Kiddie, G.A., van Ooyen, A. and Graham, B.P. (2004) Biologically plausible model of growing neurites. Brain Inspired Cognitive Systems (BICS), Stirling, U.K.

- Kiddie, G.A., van Ooyen, A. and Graham, B.P. (2004). A mathematical model of MAP2 dependent elongation and branching in growing dendrites. FENS, Lisbon, Portugal. 


\section{CHAPTER 2}

\section{Background}

\subsection{Neuron composition}

A neuron is an electrically and chemically active cell in the nervous system that acts as a collator and decision maker. Information is gathered throughout the cell, processed and perhaps transmitted to the other end. The behaviour of these cells are consistent, despite their morphological differences. A classical neuron consists of three distinct elements: the main cell body known as the soma; the information gathering part known as the dendrites; and the information delivery part known as the axon, though other neuronal types differ. This thesis concentrates on the soma minimally, the dendritic tree widely, and the axon not at all.

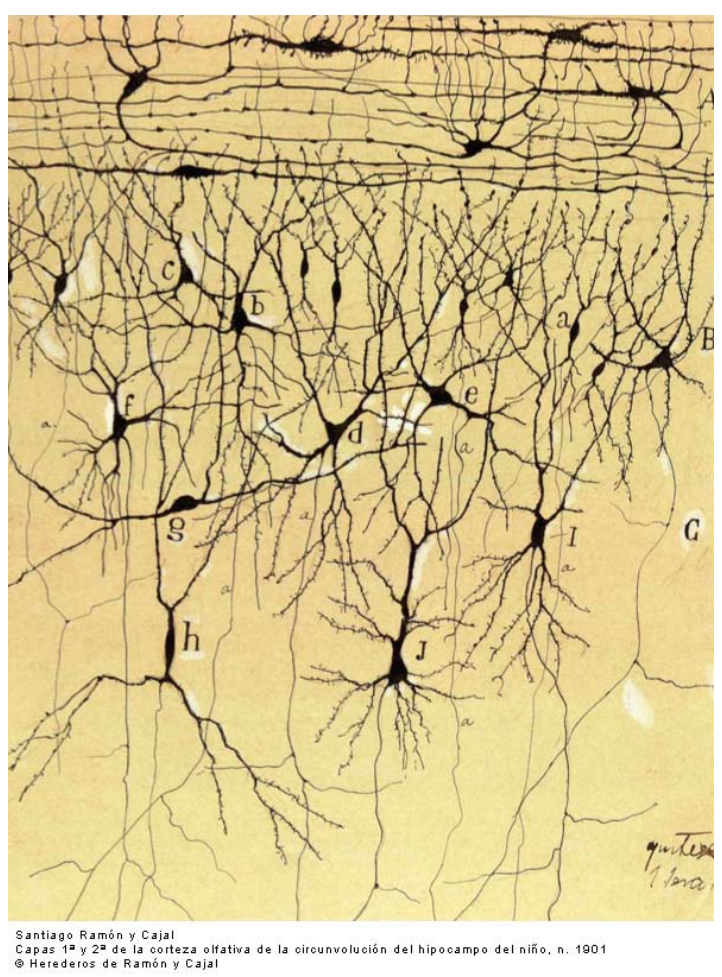

FIGURE 1. NEURONS AS ORIGINALLY DRAWN BY CAJAL (CAJAL, 1901) 


\subsubsection{Soma}

The soma is the central body of the neuron. It is the initial part of the cell from which all neurites are initiated. It includes the cell nucleus which contains the DNA for the cell among other markers and proteins necessary to differentiate the cell. The cell has a cell membrane which protects it from the external environment. The membrane is a lipid bilayer that allows the infusion of certain chemicals while blocking others, and allowing the outfusion of certain chemicals while retaining others. The cell body is also electrically sensitive as axons from other neurons may make contact with the cell body to deliver action potentials. The cell also contains mitochondria in order to power the cell, including its development. A single neuron takes a comparatively large amount of power to work which is why the brain requires so much oxygen in order to function. In a human, despite being only $2 \%$ of the body's total weight, it requires $20 \%$ of its oxygen consumption. (Magistretti et al, 2000)

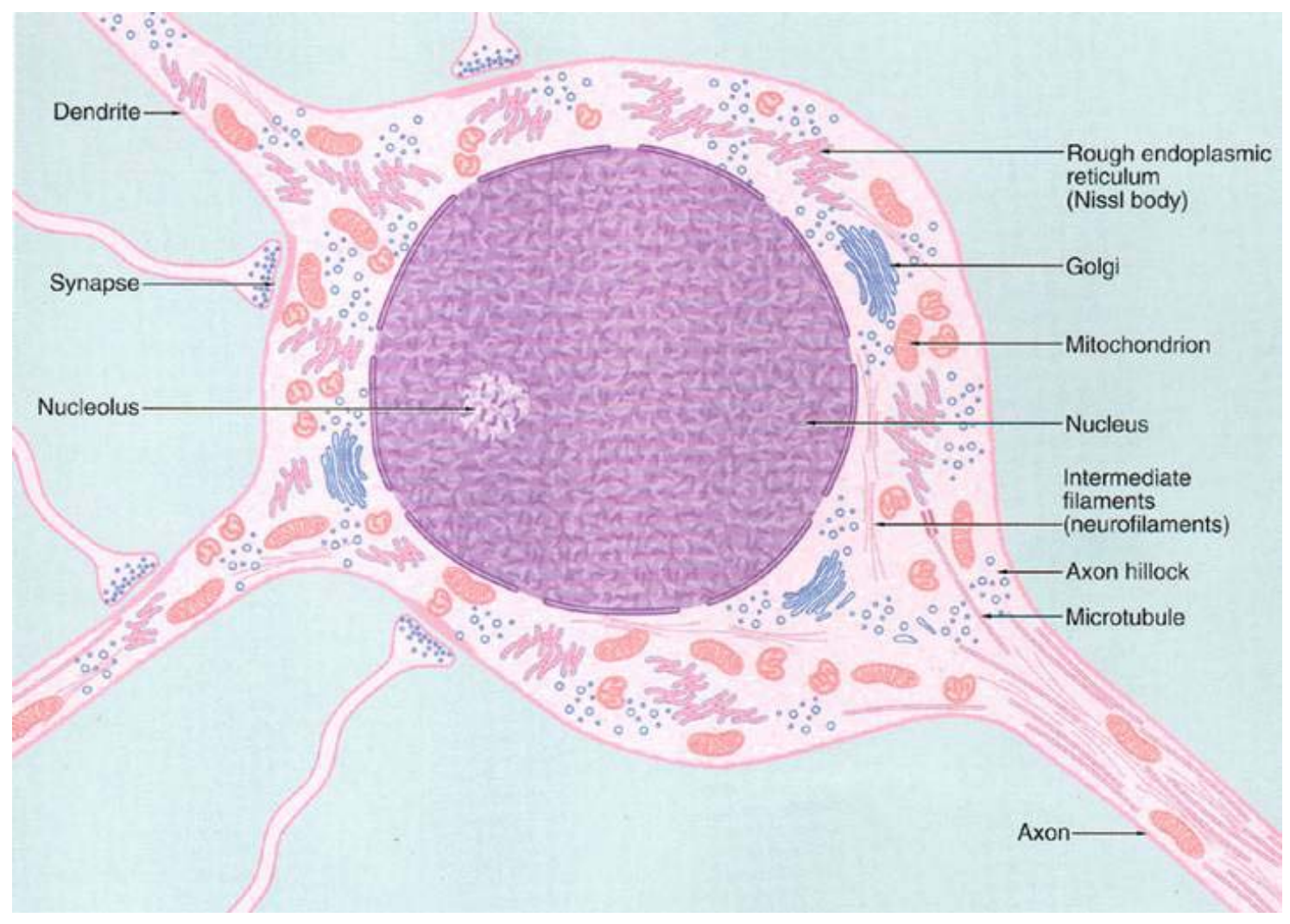

FIGURE 2. CELL STRUCTURE (VANDERBLIT UNIVERSITY MEDICAL CENTRE) 
The soma includes many processes which are important for the development of the cell as a whole. The soma includes elements such as Golgi's apparatus which allows secretion of synthesised proteins from the cell itself. The soma allows influx of calcium as do the cell's dendrites, it also produces the tubulin and MAP-2 proteins that are then transported and diffused throughout the dendrites. These chemicals are diffused, in the cases of calcium, tubulin and MAP-2, and also actively transported in the case of tubulin. The cell body itself does not contain any stabilising structures such as microtubules, these structures start at the point the extrusions are generated from the main body of the cell. The cell body instead contains something called neurofibrils, which help maintain cell shape and integrity, these are bundled together into chains much thinner and less stiff than microtubules, called filaments. Once the cell body starts developing dendrites and an axon it does not change particularly. The shape of the cell may be distorted by new dendrites growing but the internal structure of the cell remains the same.

The soma is the most important part of the neuron. Its behaviour for example in the pyramidal neurons of a rat cerebral cortex, once fully developed, is to take the electrical impulses collated by the dendrites. If this overcomes a particular threshold at the axon hillock, the electrical signal known as the action potential is generated and propagates along the axon.

The cell as a whole also has a collection of supporting cells such as glia which provide physical support and insulation from other neurons and also encourage the initial development of dendrites and the axon during the cell's initiation. They also help provide the cell with nutrients such as oxygen in order to keep the cell alive and working correctly. Glia are in effect the glue that keeps the cells and therefore the brain functioning among their other functions.

Other than as a source for proteins to be passed around the structure, the soma plays little role in this thesis. It is always assumed to have a set size and diameter and has little further impact. 


\subsubsection{Dendrites}

A dendrite is an extrusion from the cell's soma which is used for information gathering. It is a comparatively short (compared to axons) structure which has many branches in order to maximise the volume of space is occupies in order to make contact with a large number of axons and so harvest a large number of action potentials. Axons, when making contact with dendrites, create synapses, which allow the transference of chemical or electrical signals from the neuron to another cell. In a chemical synapse, when the receptors receive a release of neurotransmitter, the post synaptic potential is created and delivered down the dendrite to the soma. An electrical synapse allows the transference of action potentials via the use of gap junctions. This is greatly simplified as dendrites can support some of the functions of axons, but none of these are related to the development of the dendrites and so are not discussed here.

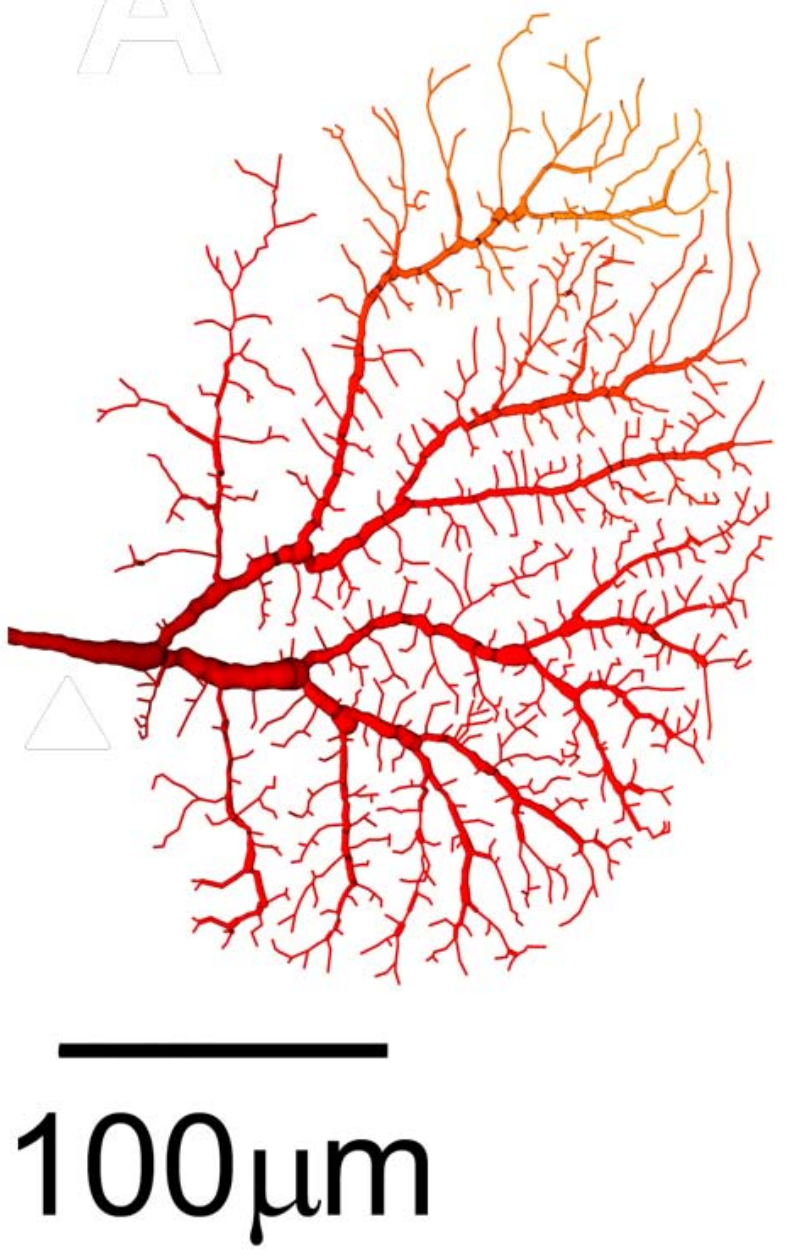

FIGURE 3. DENDRITIC STRUCTURE (CUNTZ, 2007) 
The actin cytoskeleton provides structure to the growth cone and by itself is not enough to maintain stability of the dendrite. The dendrite contains bundles of microtubules and a more flexible structure of neurofilaments which help maintain the structure and integrity. The membrane allows the influx of calcium along its length and the calcium is diffused along the length of the dendrite. The dendrite also diffuses the tubulin and MAP-2 proteins which tend to diffuse from proximal to distal ends due to the processes at work in binding the tubulin to the microtubule structure. The dendrites of neurons such as a rat's pyramidal cell also uses motor proteins in order to actively transport tubulin through the dendritic structure (Abe et al, 2004).

Dendrites can grow a variety of spines along their length, usually in order to make contact with close by axons, and so create synapses on the head of the spine. The spine is a dynamic structure which changes its shape according to the strength of the potentiation it is dealing with (along with back propagating potentiation, this helps with long term potentiation). While the spine has the same actin cytoskeleton as the rest of the dendrite and have a dynamic shape, there is no microtubule stabilisation or formation within it. This is what allows the spine to be dynamic with its shape. Certain global disorders such as Autism and age related disorders such as Alzheimers are connected to problems with spine frequency as the brain is unable to maintain as many synapses as in a healthy brain (Bhatt, et al 2009). Smooth dendrites have few spines and rather than receiving axons on spines, receive them directly on their shaft.

As the dendrite grows it branches repeatedly, forming the tree like structure that gives it its name. The tips of each of these branches have a growth cone. The growth cone has several functions including dealing with excitatory and inhibitory environmental guidance cues, moving through the substrate surrounding the cell and splitting to become separate branches. The growth cone itself has many filopodia which extrude from the cytoskeleton of the growth cone and which adhere to the substrate surrounding the cell, and are given shape by the actin filaments which make up their core. These filopodia encourage the growth cone to be motile, producing a tension which pulls the dendrite through the substrate and provides a counterpoint to the push of the microtubules. The filopodia are dynamic structures and react to the environmental cues to pull the cone in different directions causing the dendrite to turn. These 
tensions can also encourage the cone to split causing a branch. As the tip elongates and branches, the actin cytoskeleton deforms and extends in order to retain the overall shape of the cell. This is why the actin cytoskeleton is not sufficient to support the dendritic structure, it must be flexible enough to allow growth and splitting. The filopodia are not stable structures and extrude and retract during their life cycle. When a filopodia is fully extruded and adhered to the substrate, the retraction of the filopodia is what causes the tension within the growth cone. During development, the tips of the branches are therefore the most active and dynamic places in the cell.

The dendrite is then a solid core of microtubules and neurofilaments providing the stability and structure for the dendrite to retain its shape and position, surrounded by an elastic deformable membrane which allows it to elongate, branch and create connections with nearby axons.

\subsubsection{Axon}

The axon is the piece of the cell that delivers information to other cells. Synapses are formed when an axon comes into contact with the dendrites of other neurons, and the action potential which passes down the axon due to a spike generated in the soma excites the synapses to release their neurotransmitters to relay this information to the other cell's dendrites. The axon shares many characteristics with the dendrites especially with respect to how the structures are formed and the proteins involved in their construction, but also has many crucial differences in their behaviour. The development and behaviour of axons is not covered by the work in this thesis. The model presented here could be adapted for use in axonal development, but there would be a stronger emphasis on external growth factors and would use a different set of microtubule-associated proteins.

\subsection{Calcium}

Calcium is a vital component for both the development of neurites and the continued functioning of them. Calcium influxes into the cell through the cell membrane, and this occurs 
all along the surface of the cell, including the dendrites. Voltage gated calcium channels are responsible for calcium spikes in the dendrites.

Intracellular calcium affects neurite outgrowth (Kater et al., 1988; Goldberg and Grabham, 1999). Deviations in either direction from an optimal calcium concentration slow down neurite elongation and can even cause retraction. Hentschel and Fine (1994, 1996; for a review, see Hentschel and Fine, 2003) have shown that this dependence of growth on calcium can lead to the emergence of dendritic forms from initially spherical cells. In their model, local outgrowth of the cell membrane is taken to depend on the local concentration of calcium close to the internal surface of the membrane. Local calcium concentration results from influx, extrusion and diffusion. Because of the larger surface to volume ratio, the local calcium concentration at spontaneously occurring small protrusions of the cell membrane will become higher than at other points of the membrane. These protrusions of the membrane will not decay because of the existence of a positive feedback loop. As a consequence of the presence of voltage sensitive calcium channels and the influence of calcium on the membrane potential, calcium influx increases with increased localised external calcium concentration. Provided that the calcium concentration does not become too high, this will lead to continued outgrowth of the protrusions. The growth of cells in their model resembles the dynamic behaviour of growing dendrites. In both model neurons and natural neurons, initial outgrowth consists of broad, irregular extensions and short, very fine extensions of the cell membrane. Distinct processes (neurites) emerge only subsequently. These processes spontaneously form enlargements at their actively growing tips, which in turn give rise to branches. In both model and natural neurons, small processes often retract, and the extension of large processes may be punctuated by episodes of stasis or retraction.

Calcium is the mainstay of much of the growth and branching inside the neurite. It underpins much of the other work performed inside the growing neurite. The real case of interest here though, is its interaction with the other chemicals. The levels of calcium in the neurite could be used to determine outgrowth, but its interaction goes deeper than that (van Pelt et al, 2002). Calcium regulates the rate in which MAP-2 binds and phosphorylates. That is to say calcium 
does change the rate of growth and the amount of branching, but in such a subtle manner, and as only part of many different elements, it is wise to look upon it as an effecter of change, rather than the be all and end all of growth (van Pelt et al, 2002).

\subsection{Tubulin}

Tubulin is a chemical that forms the cytoskeleton of the dendrite. Tubulin is produced in the soma, and is actively transported and diffused through the length of the dendrite to the distal tips of the microtubule structures or growth cones, as dendrites have no process for synthesis of the protein. Tubulin monomers form polarised dimers comprising alpha and beta isoforms, with positive and negative ends. In the dendrite, the positive end is facing the terminal tip, and attaches to the microtubule bundles with the negative end. This addition of tubulin dimers to the end of microtubules performs growth (Maccioni, Cambiazo, 1995). Microtubules become bundled together to form the growth cone, it is bundled together to form a thick rod like structure through the middle of the dendrite. The rate of addition of tubulin dimers to microtubules and the bundling of microtubules are both regulated by MAP-2.

Microtubules requires sufficient free tubulin in order to elongate An unstable microtubule structure which is starved of tubulin or MAP-2 with which to bind will suffer a catastrophe and the tubulin will start to disassemble from the microtubules. This free tubulin will likely diffuse back down the dendrite, leaving little free tubulin in the growth cone which will cause further microtubule collapse.

\subsection{Microtubules}

Microtubules are created from the polymerised state of tubulin. These are long stick-like cylindrical structures which form a spine like central core to the extrusions of the neuron, being present in both the dendrites and the axon. Tubulin dimers polymarise negative end to positive end to create protofilaments, thirteen of which are connected in a spiral to initially create a 
single microtubule, which can then extend through the addition of further protofilaments. Microtubules are vital to the formation of dendrites as they are able to withstand the pressures of pulling and pushing which are generated by the effects of growth, and have many proteins which bind to this structure in particular the motor proteins such as kinesin and dynein which actively transport other chemicals such as tubulin within the dendritic structure.

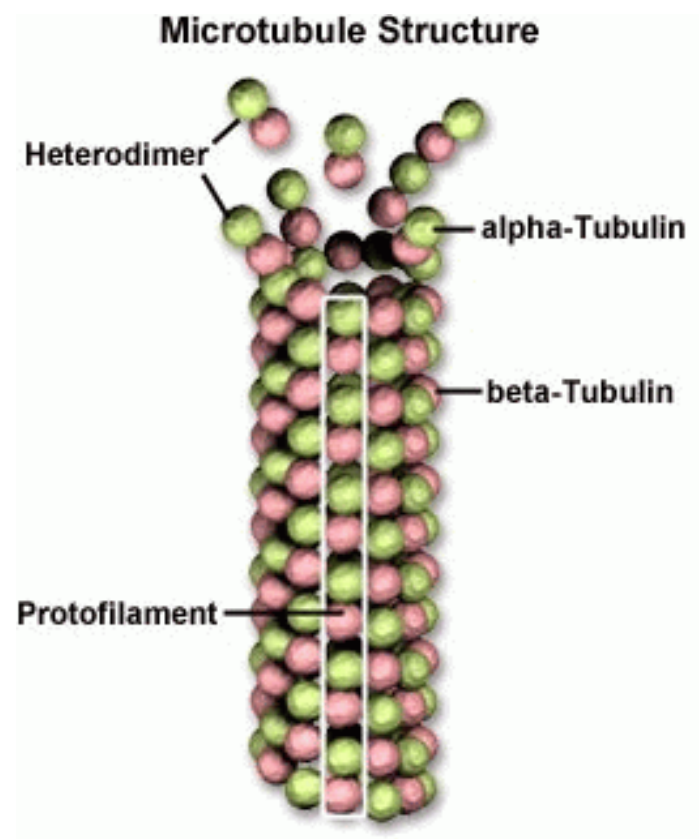

FIGURE 4. STRUCTURE OF MICROTUBULES(BAULIN, 2005)

Microtubules are polarised with the negative end sited toward the main cell body and the positive end toward the distal tip. Microtubules are formed by the attachment of protofilaments at the distal tips of dendritic terminals. This process is stabilised by the presence of MAP-2 in its bound state which helps bind the tubulin to the microtubule structure. The tubulin is attached in a ring, end to end to produce the cylindrical structures that make the microtubule, the tubulin is added in a polarised order to keep the positive and negative ends consistent (see figure 4). The microtubules provide an additional pressure to the dendrite's growth cone by pushing the actin tip internally. This process can be involved in directing the growth cone away from inhibitory cues by applying this internal pressure. Branching of the growth cone can be facilitated by the destabilisation of the microtubules, bonds tightly holding the bundles of microtubules are relaxed, allowing these microtubules to separate and move in different 
directions if the conditions are right (Kobayashi, Mundel, 1997). The microtubules splay out in these branching scenarios having gone through some internal reorganisation.

Microtubules are essentially dynamic structures, without the presence of bound MAP-2 and a plentiful supply of tubulin, the microtubule can retract, tubulin being freed from the bound structure and decaying naturally. This results in a constant construction and destruction balance within the dendrite. When a microtubule is starved of the necessary proteins for growth this process takes place, and in extreme cases the entire extrusion can collapse back into its parent, this being known as an Armageddon event (Janulevicius et al, 2003). Microtubules can also be stabilised by external events such as synapse formation. These events appear to release a certain set of proteins which helps maintain the formation of microtubules and reduces competition for resources with other parts of the dendritic structure.

\subsection{MAP-2}

MAP is a collection of chemicals known as Microtubule Associated Proteins, and MAP-2 is a specific chemical inside this family. The main purpose of MAP-2 in the growing neurite is to determine how likely growth or branching is to be performed. MAP-2 dictates these factors by the way in which it affects tubulin (Audesirk et al, 1997). MAP-2 can exist in one of three states, unbound, bound to microtubules (dephosphorylated) and phosphorylated. The rates of transitions between these three states are controlled by the amount of calcium present in the growth cone. The figure 5 shows how the three states relate to each other and how calcium affects these state changes. Dephosphorylated MAP-2 favours growth as it promotes the bundling of microtubules and the addition of new tubulin to the ends of microtubules, creating long microtubules and forcing the neurite to create more space by becoming longer. Phosphorylated MAP-2 is more likely to create branching conditions as the microtubules' bindings are relaxed, are spaced further apart and are therefore easier to be forced apart by the correct conditions. See 3.1 for the equations governing how these states interact with each other. 


\subsubsection{Unbound MAP-2}

Unbound MAP-2 is created in the soma and diffuses down the dendrites into the growth cone. Unbound MAP-2 does not have any effect on the branching or elongation rates. Once in the growth cone, it loses a certain amount of itself to decay, a certain amount of itself gets altered into its bound state in order to bind tubulin to the present microtubules. Further unbound MAP2 results from MAP-2 bound to microtubules being released by the present calcium.

\subsubsection{Bound (Dephosplorylated) MAP-2}

Bound (dephosphorylated ) MAP-2 is synthesised from the other two states of MAP-2. It loses some of itself to decay, it loses some of itself to being unbound and it loses some of itself into being phosphorylated. It gains from the unbound state and it gains from the phosphorylated state.

\subsubsection{Phosphorylated MAP-2}

Phosphorylated MAP-2 is synthesised from the other two states of MAP-2. It loses some of itself to decay, it loses some of itself to being unbound and it loses some of itself into being dephosphorylated. It gains from the unbound state and it gains from the dephosphorylated state. The effect calcium has on these is the rate at which MAP-2 is converted between its phosphorylated and dephosphorylated states. A cell which allows calcium to affect the rate of change in one direction over the other will have a greater degree of effect over the overall morphology of the dendritic structure. 


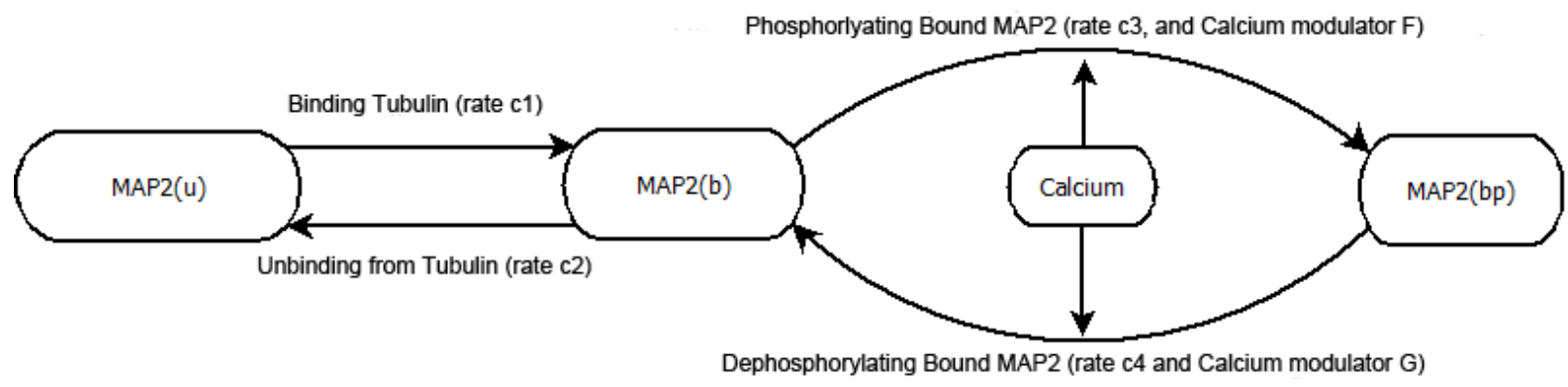

FIGURE 5. MAP-2 STATES AS REGULATED BY THE RATE CHANGE CONSTANTS (C1 - C4) AND THE RATE CHANGE MODULATORS F AND G WHICH ARE FUNCTIONS OF CALCIUM THAT QUANTIFY HOW THE CALCIUM CONCENTRATION AFFECTS THE PHOSPHORYLATION AND DEPHOSPHORYLATION RATES.

MAP-2 is not the only protein which has an effect over the branching and elongation rates. Experimental results shows many expressed proteins having a greater or lesser effect through over or under expression (Abe et al, 2004; Cline 2001). Increasing the amount of kinesin proteins to speed up the transfer of tubulin from the soma to the terminal would increase the overall length of the dendrites for example.

\subsection{Elongation}

Elongation in dendrites is controlled by the processes and mechanisms described by tubulin and microtubules.

\subsection{Branching}

Branching in dendrites is controlled by two separate mechanisms. Growth cone branching is the main form of branching under developmental conditions. This is the process covered above where the growth cone is under enough stress due to internal pressures from microtubule formation and external pressures caused by filopodia reacting to environmental cues. Interstitial branching occurs in established dendrites, where the shaft initially extrudes a filopodia, presumably in response to a new environmental cue and in a process not yet fully understood, and this filopodia will either retract back into the dendritic shaft or proceed to develop into a 
full growth cone at which point it is treated as a regular branch. An excess of tubulin at this point seems to be the key to the formation of the growth cone, as it appears that microtubules supporting the initial filopodia must fragment from the main body of the dendrite, start attracting and attaching tubulin and support the proto-growth cone fully. The environmental cue which causes the filopodia to form may also be responsible for the destabilisation of the actin cytoskeleton and the microtubule formation.

\subsection{Diameter changes}

The diameter of a segment of a dendrite changes over time. It initially starts thin, tapering towards the distal end. As the dendrite grows and branches, it gets gradually thicker towards the proximal end, while the distal end remains tapered and thin. The result of this is that for each branch an intermediate segment is attempting to support, the intermediate segment must thicken to support it. The thickening is not directly related to the number of branches it supports as asymmetrical dendrites show that if one branch is much thicker than the other, the supporting intermediate segment is thick enough to support the more dominant branch, the less dominant branch does not appear to require any additional thickness from the supporting intermediate segment (Cuntz et al, 2007).

\subsection{Models of neurite growth}

\subsubsection{Models}

In the following sections we will review mathematical models of neurite outgrowth that attempt to capture biophysical aspects of neurite initiation, elongation and branching. Typically such models concentrate on a particular aspect of neurite outgrowth, with an emphasis either on the intracellular or extracellular biophysics. Such a classification of the models is given in Table 1. 


\begin{tabular}{|c|c|c|}
\hline & Intracellular & Extracellular \\
\hline Initiation & $\begin{array}{l}\text { Hentschel and Fine }(1994,1996) \\
\text { Samuels, Hentschel and Fine (1996) }\end{array}$ & \\
\hline Elongation & $\begin{array}{l}\text { Li, Qin and Wang (1994) } \\
\text { Miller and Samuels (1997) } \\
\text { McLean and Graham (2004) } \\
\text { McLean, van Ooyen and Graham } \\
\text { (2004) } \\
\text { van Ooyen, Graham and Ramakers } \\
\text { (2001) } \\
\text { van Veen and van Pelt (1994) }\end{array}$ & $\begin{array}{l}\text { van Veen and van Pelt (1992) } \\
\text { Li, Qin and Wang (1992) } \\
\text { Li, Qin and Wang (1995) }\end{array}$ \\
\hline Branching & $\begin{array}{l}\text { Hely, Graham and Van Ooyen } \\
\text { (2001) } \\
\text { Van Pelt, Graham and Ulyings } \\
\text { (2003) } \\
\text { Zubler and Douglas (2009) }\end{array}$ & $\begin{array}{l}\text { van Veen and van Pelt (1992) } \\
\text { Li, Qin and Wang (1992) } \\
\text { Li, Qin and Wang (1995) } \\
\text { Eberhard et al (2006) } \\
\text { Koene et al (2009) } \\
\text { Cuntz et al (2010) }\end{array}$ \\
\hline
\end{tabular}

Table 1. Model Classification by Type

Intracellular models typically only seek to reproduce realistic neurite topologies, in terms of the number of terminals and the lengths of terminal and intermediate segments. The actual shape of the neurite in two- or three-dimensional space is not considered. An exception is the model of neurite initiation by Hentschel and Fine $(1994,1996)$ which generates neurite outgrowth in a two-dimensional plane due to intracellular instabilities. Extracellular models also produce neuronal shape. The models reviewed here only consider two-dimensional environments. Modelling growth in vivo would require a three-dimensional environment. This has been 
achieved in the non-biophysical, stochastic L-system work of Ascoli and colleagues (Ascoli 2002) Eberhard (Eberhard et al, 2006), Koene (Koene et al 2009) ,Cuntz et al (2010), and Zubler and Douglas (Zubler and Douglas 2009), but presents great computational demands for biophysically-based models.

In addition, models of neurite outgrowth can be classified according to the mathematical techniques they employ. Typically either ordinary differential equations (ODEs) or partial differential equations (PDEs) specify the growth model. A coupled set of ODEs specifies the dynamics of quantities at particular points within the growing neurite, typically the cell body, branch points and terminals. PDEs specify values at all spatial locations. In either case, results are produced by numerical integration using finite difference schemes. The proper formulation of appropriate finite difference schemes is complicated by the changing morphology of a growing neurite, leading to free and moving boundary problems. This led Hentschel and Fine (1994, 1996) to use a cellular automata approach to designate regions as being inside, outside or forming the membrane of a neuron. Consideration of numerical techniques is given below. Certain models are also amenable to analysis (Hentschel and Fine 1994; van Veen and van Pelt 1994; McLean and Graham 2004). Classification of models according to their mathematical formulation is shown in Table 2. 


\begin{tabular}{|l|l|}
\hline ODE & \begin{tabular}{l} 
Samuels, Hentschel and Fine (1996) \\
Li, Qin, and Wang (1992) \\
Li, Qin, and Wang (1994) \\
Li, Qin, and Wang (1995) \\
Miller and Samuels (1997) \\
van Ooyen, Graham and Ramakers (2001) \\
van Pelt, Graham and Ulyings (2003) \\
van Veen and van Pelt (1992) \\
van Veen and van Pelt (1994) \\
Zubler and Douglas (2009) \\
Eberhard et al (2006) \\
Koene et al (2009) \\
Cuntz et al (2010) \\
\hline PDE
\end{tabular} \\
\hline
\end{tabular}

Table 2. Model Classification by Mathematical Techniques

\subsubsection{Compartmental Modelling}

Mathematical modelling of neuronal properties often involves calculating quantities that vary over the spatial extent of the neuron. This is usually achieved by a one-dimensional spatial 
discretization of the tree structure formed by dendrites and the axon. When the quantity of interest is membrane voltage, this method has become known as compartmental modelling (see e.g. Koch and Segev 1998). Techniques for specifying an appropriate compartmentalization (or discretization) for accurate calculation of voltage are well established.

Models that deal with the biophysics of neurite outgrowth typically are concerned with the concentration of chemicals, both intra- and extracellularly, that determine growth. Intracellular PDE models use a similar one-dimensional discretization to compartmental modelling of voltage. A difficulty arises, however, in that the cell morphology changes over time, necessitating continual respecification of the discretization.

\subsubsection{Fixed compartment number}

Numerical solution of a PDE model of neurite elongation by means of finite differences (McLean et al 2004) yields a compartment model which contains a fixed number of compartments, regardless of the neurite's length. This is achieved via a spatial transformation of the model so that spatial values are always solved over a [0,1] length domain. Values are calculated at $\mathrm{N}$ different points within this domain, where $\mathrm{N}$ is the number of compartments. As the actual neurite length changes over time, so the real spatial locations (or, equivalently, the size of the compartments) of the $\mathrm{N}$ values changes, as illustrated in Figure 6. 

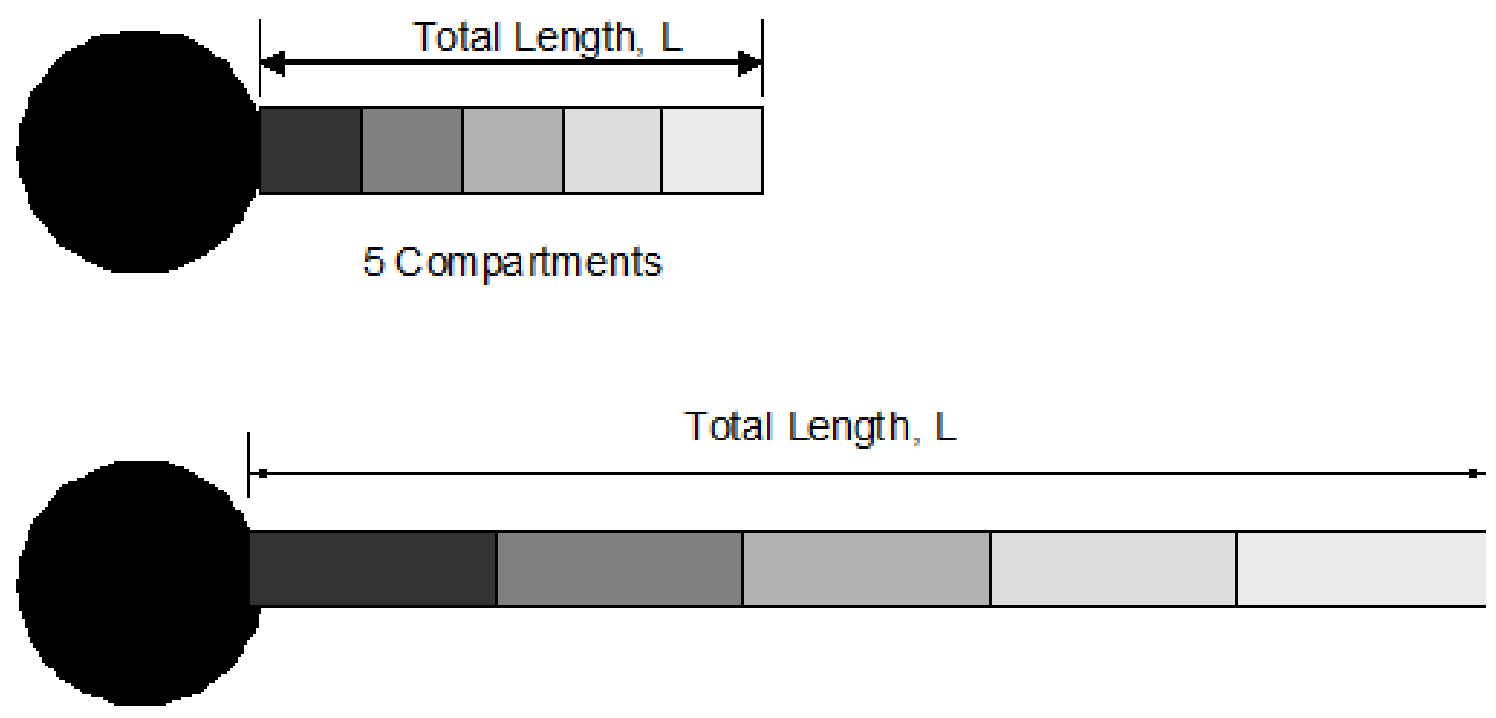

FIGURE 6. DISCRETIZATION OF ELONGATION MODEL. (TOP) EARLY TIME. (BOTTOM) LATER TIME.

For numerical stability it is important that the number of compartments, $\mathrm{N}$, is sufficient for the discretization of the maximum expected length. This may be an unnecessarily large number for the early stages of growth when the length is short.

The fact that the compartments are not positionally static may also be problematic for modelling particular situations. For example, modelling synapse formation during neurite outgrowth and the subsequent response of the neuron to synaptic input requires that the exact spatial location of the synapse be stationary.

\subsubsection{Fixed Compartment size}

An alternative discretization scheme keeps the size of most compartments fixed and allows the addition of new compartments. A number of variations on how neurite elongation and branching are handled with such a scheme are possible (Graham and van Ooyen, 2001). The most numerically satisfactory approach is illustrated in Figure 7. Here, all but the single compartments immediately preceding branch terminals (growth cones) remain of fixed size, $d x$. Growth is handled by elongating these preceding compartments. When their length exceeds $2 * d x$ they are divided into two compartments, the most proximal of which is given the fixed 
length $d x$, with the distal compartment retaining the remaining length. It is also the one that continues elongating.

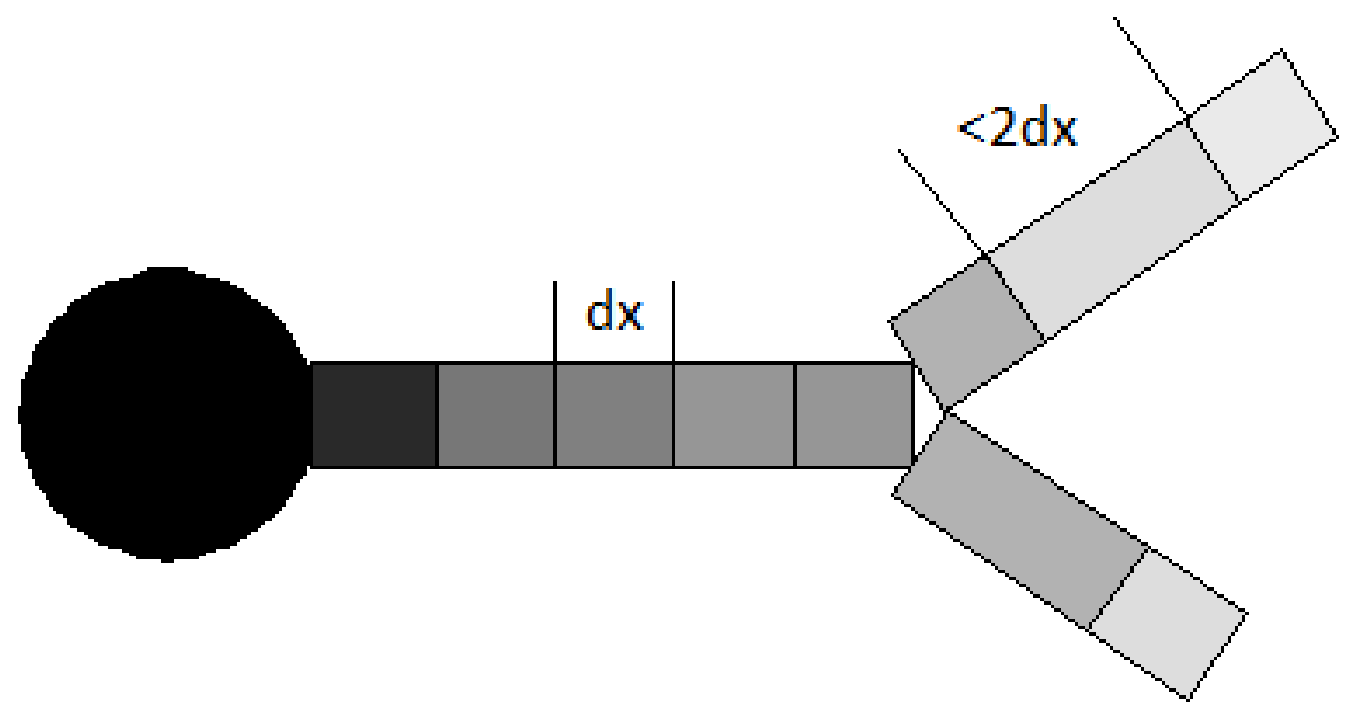

FIGURE 7. FIXED SIZE COMPARTMENT MODEL

In this scheme most discrete points at which chemical concentrations are calculated are positionally static. This may be advantageous for modelling neurite interaction with the external environment, including contact and synapse formation with other neurites. However, specifying a single compartment size, $d x$, is inefficient. A size that is small enough for accurate calculation in short neurite branches may lead to older, longer branches being described by an unnecessarily large number of compartments with a resultant high computational load for numerical simulation. Both this fixed compartment size and the fixed compartment number approaches to spatial discretization thus are not optimal in particular situations, but both provide practical solutions. The fixed size scheme has been used to implement detailed elongation and branching models that rely on accurate calculation of chemical concentrations in terminal compartments (Hely et al, 2001; Kiddie et al 2004, 2005).

\subsubsection{Elongation models}

A family of models has considered neurite elongation as determined by the production and transport of tubulin along the neurite and subsequent microtubule assembly at the neurite tip 
(van Veen and van Pelt 1994; Miller and Samuels 1997; van Ooyen et al 2001; McLean and Graham 2004; McLean et al 2004).

The work of Miller and Samuels (1997) attempted to explain the steady state or maximal lengths in axonal growth under the so-called slow transport mechanism. The hypothesis was that axonal length is limited by the distance degrading cytoskeletal proteins can be transported. In a simple model of protein transport, Miller and Samuels (1997) described the mechanisms of protein flux and protein degradation with a single ODE which was subsequently considered only in the steady state. They used a specifically simple form for the flux being the product of the average, or apparent, transport velocity and the protein concentration. Arguing that the protein concentration was constant along the length of a mature axon, they were able to find maximal lengths in the steady state. Their model was verified against a number of data for slow transport in various animal axons. The simplicity of their model even allowed them to track, with some success, the peak of a labelled protein distribution taken from the work of Hoffman and Lasek (1975).

The limiting effects of tubulin transport on the dynamics of neurite elongation have also been investigated in simple ODE models (van Veen and van Pelt 1994; van Ooyen et al 2001). A constant elongation rate results if tubulin transport is by diffusion only and tubulin degradation is sufficiently slow that it can be neglected (van Veen and van Pelt 1994). Variation in transport and particularly in microtubule (dis)assembly rates between neurite branches leads to competitive growth in which particular neurites may obtain more of the finite tubulin resource. A dominant branch can restrict outgrowth of other branches. If a dominant branch stops growing, for example due to forming a synaptic connection, the tubulin concentration in the other growth cones increases, thus facilitating the outgrowth of previously dormant branches (van Ooyen et al 2001).

In a generalisation of the above models, McLean and Graham (2004) took a conservation law approach for the concentration of tubulin. Mechanisms of active molecular transport, diffusion and degradation led to a spatio-temporal continuum PDE model for the bulk or average tubulin concentration and neurite length. Under this model, the growth of a neurite begins with the 
production of tubulin at the cell body, its displacement to the growth tip followed by its polymerization onto the microtubules at the growth tip. The model is mathematically challenging since it is of the moving boundary type. A spatial transformation that introduced nonlinearities to the tubulin governing equation rendered the growth domain fixed. McLean and Graham (2004) then sought steady state solutions which lead to the derivation of a transcendental equation for the steady state length. Having identified a key parameter grouping, they found three different regimes for small, intermediate and large steady state neurite lengths. It was established that a neuron could easily regulate the extent of its neurites by simply modulating the tubulin production at the soma relative to the active transport / degradation fraction. An eigenvalue analysis and stability analysis of the unsteady problem is currently underway to ascertain the stability of these steady state solutions. Numerical solutions of the model (McLean et al 2004) reveal that oscillations in neurite length may appear in the transition from small to large growth.

In the models described above, neurite elongation due to external effects, such as tension on the growth cone, are not considered. Models of branching due to tension on filopodia also determine elongation rates on the basis of this tension ( $\mathrm{Li}$ et al 1992, 1995; van Veen and van Pelt 1992). Li et al (1994) examined a system of growth cone locomotion based upon actin polymerization and growth cone adhesion. This is similar to microtubule assembly in the trailing neurite, but concentrated upon the construction of the actin cytoskeleton in the growth cone. Filopodia are not explicitly modelled. The leading edge of the growth cone is assumed to adhere to the substrate. Assembly of actin filaments at the leading edge and disassembly at the transition from the growth cone to the neurite results in caterpillar-style forward movement of the growth cone due to traction forces.

Related models are more concerned with the direction of neurite outgrowth, rather than the mechanisms generating elongation. Of particular importance here is understanding how cues in the environment can guide axons over long distances to their appropriate targets (Hentschel and van Ooyen 1999; Aeschlimann 2000; Goodhill 2003). 
Mathematical models can also be used to examine the microscopic phenomena underlying these growth models. Smith and Simmons (2001) consider scenarios for motor-assisted (active) transport of organelles. The microscopic dynamics of microtubule assembly have been modelled (Odde 1997; Pedigo and Williams 2002). Janulevicius et al (2003) determine how the volume of a growth cone influences microtubule assembly within the cone.

\subsubsection{Branching Models}

Several papers consider neurite branching as a result of external tension applied to the growth cone (Li et al 1992, 1995; van Veen and van Pelt 1992). In these models, filopodia extending from the leading edge of the growth cone adhere to the substrate in directions determined by attractive or repulsive cues in the environment. If all the filopodia pull in the same direction, the neurite elongates. However, filopodia on opposite sides of the growth cone may find attractive cues in different directions, resulting in tension on the growth cone. If this tension overcomes a pre-set threshold then branching occurs by splitting of the growth cone. The tension threshold is implicitly determined by the state of the actin cytoskeleton within the growth cone. These models differ in detail concerning the specification of filopodia and cues in the external environment. Li et al (1992, 1995) also introduce an implicit intracellular mechanism by which the outgrowth of a neurite inhibits the growth of other neurites from the same cell. This could be due to competition for available tubulin, as explicitly investigated in the elongation models described above (van Veen and van Pelt 1994; van Ooyen et al 2001).

Branching is also in part determined by the construction and stability of the microtubule cytoskeleton in the trailing neurite. Several models have investigated the neurite morphologies that result if branching is rate-limited by the availability of a resource, such as free tubulin, at neurite terminals (Graham et al 1998; van Pelt et al 2003) If this resource is produced in the cell body and is transported by diffusion and active transport to the terminals, the branching rate is modulated both with the number of terminals in the growing tree and their centrifugal order (number of branch points between a terminal and the cell body). This is similar to the 
branching dependencies of real neurites, revealed by statistical models of neurite outgrowth (van Pelt et al 1997; van Pelt and Uylings 1999).

A model of elongation and branching as modulated by the effects of MAP-2 on the construction of the microtubule cytoskeleton has been proposed in Hely et al (2001). In this model, the rates of elongation and branching were in part determined by the rate of binding, unbinding and phosphorylation of MAP-2. Binding of unphosphorylated MAP-2 to microtubules at a neurite tip increased the rate of microtubule assembly and stabilised the microtubule bundles, leading to faster elongation and a lower rate of branching. Phosphorylation of the MAP-2 disrupted its stabilising function and promoted branching. The rate of phosphorylation was controlled by the local calcium concentration. Changes in calcium concentration during outgrowth, and the precise relationship between calcium and MAP-2 phosphorylation, determined the resultant branched tree morphology. 


\section{CHAPTER 3}

\section{New Elongation and Branching Model}

Here we describe a new model that incorporates the effects of MAP-2 on the construction of the microtubule cytoskeleton (Kiddie et al 2004, 2005). This effectively combines the microtubulebased elongation models with the MAP-2 model of Hely et al (2001). Tubulin and MAP-2 are produced in the cell body and transported by active transport and diffusion to the neurite tips. calcium influx occurs along the length of the neurite and calcium can diffuse internally. Neurite elongation is a function of the microtubule assembly rate, which depends on the available tubulin and is modulated by bound (unphosphorylated) MAP-2. The terminal branching probability is a function of the relative amount of phosphorylated MAP-2 to dephosphorylated MAP-2.

Hely's model only considered MAP-2 and calcium in a similar set of equations as to those below. His elongation and branching equations only considered MAP-2 concentrations, and have been altered to include tubulin in the elongation equations, and the branching equation has been altered to allow finer interaction between the phosphorylated and dephosphorylated states. The equations for each of the three chemicals, tubulin, MAP-2 and calcium, are given below. They are presented in the form of a first-order discretization of the underlying PDE model that is equivalent to the compartment structure illustrated in Fig. 7. The model neurite is divided into the soma, the intermediate compartments and the terminal compartments. The soma has a simple production/influx-transport-decay structure for each chemical. The intermediate compartments have a transport in - transport out - decay structure. Terminal compartments are more complicated. The calcium retains its influx-diffusion-decay structure. Tubulin is still transported and may decay, but is now also affected by assembly and disassembly to and from microtubules. The unbound MAP-2 still diffuses and decays, but may now bind to microtubules. The bound MAP-2 is affected by decay and its phosphorylation rate, which is a 
function of calcium. The conversion rate constants c1 - c4 are set relative to a unit diffusion rate $(D=1)$ to provide unitless concentrations that are of order 1 .

The rate at which a neurite in the model elongates is a function of the microtubule assembly, which is dependent upon the amount of free tubulin and the amount of bound MAP-2, and the disassembly rate which is a fixed decay rate.

\subsection{Equations}

All the model equations are of the form of the PDE for diffusion in one dimension, with additional active transport, production and decay terms. For example, tubulin concentration along a neurite, determined by diffusion, active transport and decay, is given by

$$
\frac{\partial T}{\partial t}=D \frac{\partial^{2} T}{\partial x^{2}}-\eta \frac{\partial T}{\partial x}-\delta T
$$

Here we give the equations in their compartmental ODE form to show how the system was solved. Explicit Euler integration was used.

\section{Soma}

$\underline{\text { Tubulin }}$

$$
\begin{aligned}
& \frac{d T_{0}}{d t}=P+\frac{\hat{D}\left(T_{1}-T_{0}\right)}{d x}-\eta T_{0}-\delta_{t} T_{0} \\
& =\text { Production + Diffusion - Active Transport - Decay }
\end{aligned}
$$

\section{Unbound MAP-2}

$$
\begin{aligned}
& \frac{d U_{0}}{d t}=S+\frac{\hat{D}\left(U_{1}-U_{0}\right)}{d x}-\delta_{u} U_{0} \\
& =\text { Production + Diffusion - Decay }
\end{aligned}
$$


Calcium

$$
\begin{aligned}
& \frac{d C_{0}}{d t}=I+\frac{\hat{D}\left(C_{1}-C_{0}\right)}{d x}-\delta_{c} C_{0} \\
& =\text { Influx + Diffusion }- \text { Decay }
\end{aligned}
$$

\section{Intermediate}

$\underline{\text { Tubulin }}$

$\frac{d T_{i}}{d t}=\frac{\hat{D}\left(T_{i+1}-T_{i}\right)}{d x}+\frac{\hat{D}\left(T_{i-1}-T_{i}\right)}{d x}+\eta T_{i-1}-\eta T_{i}-\delta_{t} T_{i}$

$=$ Diffusion + Active Transport - Decay

Unbound MAP-2

$$
\begin{aligned}
& \frac{d U_{i}}{d t}=\frac{\hat{D}\left(U_{i+1}-U_{i}\right)}{d x}+\frac{\hat{D}\left(U_{i-1}-U_{i}\right)}{d x}-\delta_{u} U_{i} \\
& =\text { Diffusion - Decay }
\end{aligned}
$$

Calcium

$$
\begin{aligned}
& \frac{d C_{i}}{d t}=I+\frac{\hat{D}\left(C_{i-1}-C_{i}\right)}{d x}+\frac{\hat{D}\left(C_{i+1}-C_{i}\right)}{d x}-\delta_{c} C_{i} \\
& =\text { Influx + Diffusion - Decay }
\end{aligned}
$$

\section{Terminal}

Tubulin

$$
\begin{aligned}
& \frac{d T_{t}}{d t}=\frac{\hat{D}\left(T_{t-1}-T_{t}\right)}{d x}+\eta T_{i-1}-\delta_{t} T_{t}-\varepsilon_{t} T_{t} B_{t}+\psi_{t} \\
& =\text { Diffusion + Active Transport In - Decay - Assembly + Disassembly }
\end{aligned}
$$


Calcium

$\frac{d C_{t}}{d t}=I+\frac{\hat{D}\left(C_{t-1}-C_{t}\right)}{d x}-\delta_{c} C_{t}$

$=$ Influx + Diffusion - Decay

Unbound MAP-2

$\frac{d U_{t}}{d t}=\frac{\hat{D}\left(U_{t-1}-U_{t}\right)}{d x}-\delta_{u} U_{t}-c_{1} U_{t}+c_{2} B_{t}$

$=$ Diffusion - Decay - Binding + Unbinding

Bound (unphosphorylated) MAP-2

$\frac{d B_{t}}{d t}=c_{1} U_{t}-c_{2} B_{t}-c_{3} F B_{t}+c_{4} G P_{t}-\delta_{b} B_{t}$

= Binding - Unbinding - Phosphorylation + Dephosphorylation - Decay

Phosphorylated MAP-2

$$
\begin{aligned}
& \frac{d P_{t}}{d t}=c_{3} F B_{t}-c_{4} G P_{t}-\delta_{p} P_{t} \\
& =\text { Phosphorylation - Dephosphorylation - Decay }
\end{aligned}
$$

\section{Elongation}

$$
\begin{aligned}
& \frac{d L}{d t}=k^{E} \varepsilon_{t} T_{t} B_{t}-\psi_{t} \\
& =\text { Microtubule Assembly - Disassembly }
\end{aligned}
$$

Branching Probability

$$
\begin{aligned}
& B_{P R}=k_{B} \frac{P_{t}}{P_{t}+B_{t}} \\
& =\text { Probability of branching }
\end{aligned}
$$




\section{Calcium Rate Converters}

$$
F=\frac{C_{t}^{P^{F}}}{k_{F}+C_{t}^{P^{F}}}
$$

$=$ Phosphorylation rate modulator

$$
G=\frac{C_{t}^{P^{G}}}{k_{G}+C_{t}^{P^{G}}}
$$

$=$ Dephosphorylation rate modulator

\section{Diffusion function $(\widehat{D})$}

Since precise measurements are not available, the small molecules calcium, tubulin and unbound MAP-2 are all given the same diffusion constant, D, but this could be set differently for each.

$$
\hat{D}=\frac{D^{*} \text { average cross sectional area }}{\text { compartmental volume }}
$$

\subsection{Morphology changes}

\subsubsection{Branching}

The first form of branching, involving growth cones, is the only form of branching considered by this model. Interstitial branching due to environmental instability is not covered due to the lack of external factors being included in the model. Branch pruning, where a branch either starved of resources due to competition, or unable to support itself shrinks back into the dendritic shaft is also not considered in this model.

Branching is performed by splitting compartments into two when reaching $2 * d x$ and the creation of branches when dictated by the event that the chance was strong enough for a branching event and the random number generator hit the right values. The branching was handled by splitting the terminal compartment into four separate compartments. A new intermediate and terminal segment on the left, and a new intermediate and terminal on the right. 
This ensured that each branch had a growth cone and a preceding segment to elongate. The intermediate compartment that had done the branching had its branching strategy updated so that it could no longer branch and its diameter was updated in order to contain the new segments. This strategy did actually change the structure of the tree, unavoidable when there was the requirement for new segments to be added, and was the only place where modification of the segment behaviours occurred.

\subsubsection{Elongation}

See section 2.9.2.2 as to how the elongation process works.

\subsubsection{Diameter changes}

The model encompasses diameter changes when the model branches. Whenever the model branches, the two new daughter branches are given an initial diameter, $d=d_{l}=d_{r}$. Their parent, now an intermediate segment, has its diameter, $d_{p}$, adjusted according to a $3 / 2$ power law, such that

$$
d_{p}^{3 / 2}=d_{l}^{3 / 2}+d_{r}^{3 / 2}
$$

All segments back to the soma now have their diameters adjusted according to the same power law. This increase in diameters throughout the tree results in an increase in each compartment's volume and a subsequent decrease in concentrations of associated chemicals.

\subsection{Elements of biological background not considered by the model}

The following notes apply to biological discussion in chapter two, outlining the obvious differences between what was stated and their place within the model. 


\subsubsection{Calcium}

The only source of intracellular calcium is assumed to be due to a constant influx of calcium across the membrane. Transient changes in calcium flux due to due the electrical activity of the neuron affecting voltage-gated calcium channels are not considered. Nor is the release of calcium from internal stores.

\subsubsection{Tubulin}

The freeing of tubulin and the collapse of microtubules is not modelled here. It was attempted but the way the model was assembled meant that as soon as a branching event occurred, a single compartment was replaced with four. To conserve the amount of chemicals in the system the amount of chemicals in the compartment was split equally between the four new compartments. The result of this was a very low level of tubulin and MAP-2 in the new compartments and immediate retraction back into a single terminal. To remove this limitation, further work would be required to determine if altering the diameter of these compartments would allow the new branches to flourish, but more likely the compartment model would need to be replaced with a model which was not dependant on the concentrations of chemicals within a small fixed space.

\subsubsection{Elongation}

The model only considers outgrowth and ignores contraction. Any negative lengths encountered are discarded and instead the compartment does not change in size at all. 


\subsection{Software for Model Implementation}

\subsubsection{Computer Language}

Java was the chosen language to write the software for numerical simulation of the above branching and elongation model in for several reasons. Several other options were considered and the pros and cons are laid out below.

$\mathrm{C}++$

Pros : Fastest of the 3 languages, being natively compiled. Plenty of libraries for any advanced mathematical techniques that were required.

Cons : Least portable requiring recompilation for each OS it would be used on. Unfamiliar. Poor UI elements. High risk.

Matlab

Pros : Fast performing mathematical equations. Excellent hard coded data visualisation post run. High level of portability. High availability of libraries for mathematical modelling. Cons : Impossible to interrupt once running so data visualisation during a run was limited. Not as fast as promised. Altering the model's parameters means altering the code rather than input via a UI.

Java

Pros : Great familiarity with language. High level of portability. Excellent UI elements for during run data visualisation.

Cons : When the period of research started, Java was slower than the other runtimes, however since then, Java has become comparable to the other runtimes in terms of speed.

Initially Matlab was chosen to create the software. This was for several reasons. Initially, it was believed that a high level of mathematical integration techniques would be needed (over and above the Euler method detailed): this was later proved to be unneeded. It was shown that the mathematical accuracy of the Euler method was sufficient through repeated testing, and that the amount of additional computational effort required for these more advanced techniques was 
more than the needed mathematical accuracy. After its development certain elements that were initially discounted, such as being able to examine elements of the model during a run, became more important. Matlab was unable to offer these elements so the software was recoded in Java. This allowed several advantages over Matlab.

Initially speed was a factor, having to wait a lengthy period of time during the testing and debugging phase to get results from the program, rather than being able to programmatically pause and interrogate the program during run time was an issue, as was being unable to tell visually whether the program was behaving correctly. Being able to visually see the tree growing in real time was a major asset when it came to working out the values of the constants in the above formulae.

Once the model was verified and the values were closer to those which made sense, the visual elements were removed from the program.

\subsubsection{Data structure}

A straightforward double linked list was used to create the tree structure. Each element in the list has a pointer to its previous elements and a left and / or right branch. Each of these elements represented a segment in the elongation and branching model as detailed above. This generic approach allows a great amount of control over the structure as it is possible to change the properties of a segment without having to greatly remodel and replace swathes of the list. This would allow a large speed boost of replacement techniques and allow in the testing of the code base through mocking of objects. Mocking of an object is where a dummy object which implements the interface of a real object while having greatly simplified behaviour, usually avoiding any external effects in order to simplify testing.

These segments then had their defining characteristics applied to them. I split the behaviours up into two different areas, updates and branching strategies. 


\subsubsection{Updates}

I used the strategy pattern throughout the program, for allowing behaviours to be modified on an existing item without costly object destruction and creation. Updates fell into three categories, SomaUpdateStrategy, IntermediateUpdateStrategy, and TerminalUpdateStrategy. Each of these corresponded to the formulae outlined above and would calculate the latest set of values in the time step they were called. They did not effect any actual change, rather working out the values based on the current application and run values and passing them back to the segment.

\subsubsection{Branching}

Again, the strategy pattern was used for modifying the behaviours of the segments. Branching fell into two categories, NoBranchingBranchingStrategy, and UnbranchedIntermediateBranchingStrategy as per the branching behaviours described in 3.2.1.

\subsubsection{Initial setup}

The structure of the initial data structure was a single soma, set up with the strategy which allowed for no branching and the some update strategy. There was a single initial intermediate segment which had the strategy which allowed branching, and the intermediate update strategy. The last segment was the terminal segment, which was set up with the strategy which allowed for no branching and the terminal update strategy.

None of the segments started with any amount of any of the chemicals. This was deliberate for several reasons, there was no conclusive numbers which dictated what the starting levels should be, the variation in the starting levels had too strong an effect on the final outcome of the morphology of the tree, and the immediate ramp up in the levels of chemicals proved both enlightening as to the interactions between the chemicals, it also allowed a more natural immediate level to the chemicals. 


\subsubsection{Working process}

The process was split into a number of discrete time steps. At each time step the following process was followed. Starting at the Soma segment, the program would walk the entire length of the list, including left and right branches for branched intermediate segments. In each of these segments, the new values would be processed, but not yet applied. Once all the segments had been visited and the new values calculated (from the values from the previous time step, ensuring that the values from the current time step did not interfere with the calculations based on the previous time step), the function pointer stack would walk back, applying the new values and running the branching strategy in turn as it worked back.

\subsubsection{Design}

\subsubsection{Strategy Pattern}

The strategy pattern is a design pattern used for varying the behaviour of a software component during its runtime. This is usually achieved by using a suite of implementations of the strategy to cover different types of variation of a particular behaviour, with each conforming to a specific interface. During the initialization phase of a component its behaviour may not be known, it may be changed during its runtime, or the behaviour may depend on the outcome of other software components. By externalising these dependencies, we keep a good separation of concerns, each software component is not reliant or aware of the other, and encourages software reuse by allowing a code component to be re-used by several software components without interaction or interference.

The implementation of this pattern is achieved by other principles of writing good and clean code, that of writing to interfaces, and favouring composition over inheritance. The interface to the strategy is the only dependence the concrete object has, multiple implementations of the interface, for example a soma update implementation and a terminal update implementation, can then be written and assigned to the concrete object, without it ever requiring knowledge of the underlying implementations. 
I used this pattern in order to have a simple underlying structure of segments, unaware of what behaviours they had in terms of branching, elongation, or even the formulae used to calculate their internal state.

\subsubsection{Factory Pattern}

The factory pattern is a design pattern used to generate concrete object based on an interface implementation. It follows several good practices when writing clean and efficient code, separation of concerns, writing to interfaces, and more general object orientated programming principles. The pattern is used to create an object which may have a complicated setup process, may require knowledge which the software component which wishes the created object does not have, and may vary in its implementation depending on the circumstances. This pattern simplifies this process by removing the logic from the software component wishing to create the concrete object and placing it in a separate class. The factory itself becomes a class of Strategy pattern by my usage of it. Each factory becomes decoupled from the surrounding code, and only the interface is used by the software components which process the model. The factory becomes the object which is swapped when a concrete class of a particular type is required, as this class still requires to create the object and it is impossible to have infinite levels of redirection. At some point, something needs to call the new operator.

Using these two techniques, there are a class of good programming practices which can be used, with mocking of objects excellent testing can be created by swapping mock objects for real ones. Additional behaviours useful for debugging can be created, for example, an update strategy which also prints out the values into the console each time step. This can be managed through dependency injection rather than awkward flags or altering (commenting out) lines of code when wanting to change behaviours. 


\subsubsection{Types}

I defined several types of concrete object which would be used by the main body of the program, following proper object orientated processes. Indeed so much behaviour was externalised and decoupled, much of the main body of the program was concentrated into two loops in a single method in a single class.

An application data object which contained all the constants for the application, these were required by many parts of the application but I kept them encapsulated within a single object type. This object only had get accessors, ensuring that the values could not be altered during the course of the applications runtime. This ensured no bugs due to logic altering the values of the constants. I did not use actual Java constants as this breaks a certain amount of object orientated behaviour.

A run data object contained all the information for performing the execution of the application. This included how many times the main loop was to be performed. As I planned on gathering data on thousands of run-throughs of the application, and I didn't wish to manually start each one, the application could run multiple times. Due to the way the application was structured so as to be generally kind on the amount of memory it consumed, the application could run for ten thousand times without any degradation in performance. The run data object was also completely encapsulated so as to only have get accessors. This followed the same pattern as the application data object.

The segment object is the underlying process object. It held references to the elements either side of it in the list, or both object succeeding it if it had branched. I made the decision that all the elements in the list would have the left and right list elements for simplicity. It would have meant that segments would have required different interfaces depending on whether they had branches or not, and this would have meant requiring to rebuild more sections of the tree every time a branching or elongation event occurred which would have slowed down the application considerably. The trade off between speed and absolute Object Orientated purity was a simple one: speed was more necessary. 
I split out the data for a segment, the chemical levels, diameter, length, etc, from the underlying segment itself. This meant I could keep the new values from a time step handy without have a group of temporary variables each time step which would have increased memory usage and caused bugs. There are a few elements of the update logic that could have been shifted into this object such as checking if the value was less than zero and setting it to zero instead, but these objects were so light I kept these logic elements in the update strategies. If the data object obtained more weight complexity wise, I would move this logic there also.

The statistics object was purely for gathering the values resulting from every run through of the application, it was very light and little more than a value object.

\subsubsection{Results}

The results objects work out the statistics involved in the tree's morphology. It walks through the tree finding points of interest, namely the start and any branched segments. Using these segments it then does short walks to determine the lengths of intermediates, the lengths of terminal branches, the order involved in the branches, and so on. These values are kept rather than immediately written.

The levels of the chemicals are not stored because they are actually rather uninteresting in the pursuit of the end goal which is the morphology. Certain things can be drawn from them and the constants used to create them and this is discussed later. The collection of levels during the course of a run were also not recorded as this would have slowed down the application considerably, and if the values of the chemicals were not interesting at the end, they would not be interesting during the course of a run.

The only time this fact was not true was during the initial stages of working out reasonable values for the constants. Being able to watch the interaction of the levels, in particular the amounts of calcium and tubulin was invaluable. This required collection of these levels for display purposes, and once these constants had been positioned appropriately, the collection of the levels could be removed for actual data gathering. 
The results module gathers the statistics and retains them using the statistics type. This is very lightweight object, no more than a value object. The gatherer itself is decoupled from the rest of the application and does not interfere with any other software components during a run. The surrounding class which controls how the application should perform activates the gatherer object once a run is complete. This ensures that there is no chance to alter the data by accident during the application's run time.

\subsubsection{Output}

The output objects take the results and do something meaningful with them. That is the sole contract into this software component. This was initially set up to both write the results out into the console and to write the results to the file system. Once visual verification became less important, the writing of the results was dropped to allow the application as much speed as possible. The output writer writes the data collection of results into separate files based on the settings for the application. There was no attempt to interpret the data at this point, there are plenty of other tools to achieve analysis of the data and so recreating them in the application would have been futile and foolish. The Output is completely decoupled from the rest of the application, the surrounding main application passes the collection of results to the output software component and so maintains the principles of decoupled components.

\subsubsection{Application core}

The main application was split into two parts. The piece of the application which was responsible for setting up and controlling the run time of the application and a piece which did the Dependency Injection. The piece which controlled the run time of the application worked out how long the application should run for, contained the main two loops for the amount of runs and the time bins for the tree. It controlled the construction of the initial set up of the tree as detailed above. This construction was not farmed out into the factory object as it was immutable, the initial structure would always be same, though the underlying object would not 
necessarily be, as they were supplied by factories. This piece controlled when the results gathering was performed and controlled when the output was written. The second piece of the main application contained the root main method for the application, and set all the dependencies for the application. This is where the concrete implementations of all the factory objects were assigned to the run time. There were several variations on this class with different sets of concrete implementations as the work progressed at different times. This means I could have a version for testing sitting alongside the correct version without needing to change any of the important runtime object, the changes were isolated to a single class. This was the outcome of following all the software engineering best practices, a single class which coupled all the decoupled pieces of the application together into a coherent whole capable of generating the tree morphologies required. 


\section{CHAPTER 4}

\section{Modelling tree types}

\subsection{Data gathered from simulation}

\subsubsection{Topology}

The results the model produces can be considered in several different ways. The concentration of MAP-2 in each of its states is a possibility as this is a key indicator of behaviour within a single element of the model. However this is not deemed to be acceptable as there are no accurate figures surrounding the concentration of MAP-2 during the growth of neurites. This is a similar situation for the majority of measurable quantities in the model. Of the elements which are considered in this model only calcium can be quantified accurately without causing irreparable harm to the cell. Measuring the outwardly visible elements of the cell, length of segments, diameter, branching angles and branching ratios, can be made without damaging the cell and so these measurements are widely available.

These then become the only measure of accuracy that the model can be judged against. By taking the measurable statistics generated by the model and comparing them to the statistical information discovered through observation, we can say with a degree of certainty that the model reflects reality. In this respect, this model does not overtake any of the previous studies presented previously, though this is due to a lack of statistical information about concentrations within a cell during growth due to the limitations of current measuring techniques without causing damage to the cell being measured.

The following measurable elements were then recorded by the model during growth.

\subsubsection{Terminal Length}

The length of the terminal segments of the model are measured by taking the distance from the last branch to the end of the terminal. No assumptions have been made about the effects of a growth cone on the length of this segment, this is assumed to have a static length which is 
included in the length of the terminal, and indeed is the minimum length of the branch, without the growth cone, the terminal segment is effectively dead and should be subsumed back into the main body of the tree (Cline, 2001; Dehmelt and Halpain, 2003; Mason and Erskine, 2000), this shows shrinkage of the tree which is possible as demonstrated by the microtubule collapse (Janulevicius et al, 2003) in where an "Armageddon" event has occurred which results in the terminal being unable to maintain its form and losing the possibility of growth. This has not been implemented in the model due to the complications and lack of information over this event. This is further discussed in chapter five. Stabilisation of a branch is also not included within the model, again due to a lack of externalised event such as synapse formation.

\subsubsection{Intermediate Length}

The length of the intermediate segments of the model results are measured by taking the distance between two branch points, and between the soma and the first branch. It is assumed in the model that no elongation of these segments is possible once a branching event has occurred. In effect, these segments become static elements which are only altered in diameter due to branching events further down the tree. It is known that they can move slightly due to tensions placed upon them by terminal segments post branching, but this is also negated by the creation of a synapse on the intermediate segment. The assumption of the model is that these elements balance each other out to provide no net effect on the model. The reason this assumption has been made is due to the lack of data about the behaviour of the movement of these segments once a branching event has occurred. It should be possible for an Armageddon event in a branch to shrink it back into the intermediate segment and so turn the other branch of an intermediate segment into an extension of the former branch, making it either a terminal or longer intermediate depending on branching events further down the tree. This has also not been included due to the lack of experimental work to back up this process. 


\subsubsection{Degree of branching}

The degree of branching in the model is measured by counting the branches within the results. The level of branching was an important factor within the results as a set of results with a high amount of branching resulted in very short intermediate segments and frequently, very short terminal segments as well. This was due to the increased competition placed on the resources by the new branches.

\subsubsection{Order}

The order is the depth of the tree. Each branching point in a route from soma to terminal tip counts towards this. The highest order among the various routes is the order for the overall tree. The order has an interesting effect on the shape of the trees. Order can be zero, in which case the tree never encountered a branching event and simply grew a long single terminal. Low order number usually result in lengthy segments, both intermediate and terminal as growth continues but the branching events are infrequent. High ordering results in shorter segments, both intermediate and terminal as repeated branching events does not allow any particular segment to grow with any particular regularity and the vast amount of structure the tree is attempting to support restricts the amount of resources the tree has available to it overall.

\subsubsection{Path Length}

The distance of a path is taken by measuring the distance from the soma to the tips of a terminal segment. This is mainly restricted by the amount of elongation possible, branching rarely restricts the levels of growth. Altering the amount of chemicals which determine elongation is key to altering the length of the paths through the tree, lowering the rate at which they enter the system lowers the overall rate of growth, upping the rate of entry makes the tree much more energetic. 


\subsubsection{Overall length}

The overall length is the total of the all the path lengths. This is then obviously directly linked to the amount of branching events that occur as every terminal in the tree means another path to add to this length. This is only indirectly related to the order, as the order determines how deep the tree is and the higher the order the more branching must have occurred, but the two are not entirely linked, a deep tree with few branches other than those off a main trunk is entirely possible to create.

\subsubsection{Topology}

The model then generates a two dimensional tree which represents the tree as it would appear in-vivo if it had been taken and flattened for observation. The model frequently creates overlapping branches in the view, but this is nothing more than cosmetic, there is no effect on the calculations performed on each of the segments even though they are considered by the viewer to share the same "physical" space. The statistical results do not come from the view but instead the model underlying the view. This is another area where a restriction has to be noted, branches are not only competing for resources, they are also competing for space within the same volume. External forces due to squeezing or available space is not considered within this thesis.

\subsubsection{The results gathering process}

The results can be gathered in a variety of ways allowing for real time gathering of information during the runs which is useful for debugging both the model along with the software, and also for allowing interesting insights into the model as it grows as it is able to display the relative levels of concentration of each of the chemicals, allowing a real time picture of where the fiercest competition for resources is at any one time. However, the mode used to generate large numbers of results forgoes any internal data gathering, purely walking the tree once the generation is complete in order to generate the necessary topology statistics. 


\subsection{Parameter values to give different types}

Initial results with this model illustrate that it can produce a variety of different types of neuritic tree morphology with changes in parameter values. There are three sets of parameters demonstrated in this section: an initial set and two modified sets that generate alternative tree topologies (Table 5).

The model is simulated by calculating the concentration of each chemical in all compartments at each time step, and performing compartment elongation and branching as necessary, using the fixed compartment size approach (see Compartmental modelling section). All chemical concentrations start at zero. Simple first-order Euler approximations over time and space are used to discretize the equations, as shown previously.

There are many ways to change the morphology of the tree all related to changing the constants which govern the interactions between the chemicals within the model. The initial approach is to alter the rate constants to produce the similar style of trees to previous works. Changing the values of other constants included in the model have various other effects upon the resulting tree. It can be shown that a high level of control over the general shape of the tree is possible. Exact control is impossible due to the random and disruptive nature of branching events, this is mitigated in these results by showing the frequency of values rather than a single set.

\subsection{Different tree types}

Neurons produce drastically different dendritic arbours, depending on the type of neuron that is generating the dendritic structure, which region of the brain the cell is developing in, and a multitude of environmental cues. Generally neurons are classified by the morphology of their dendrites, and the model is able to produce three different types of dendritic structures starting with a structure with long terminal segments such as those seen in dendrites of pyramidal neurons in rats, dendritic structures with equal segment lengths, such as Purkinje cells, and dendritic structures with long intermediate segments such as granule cells. 


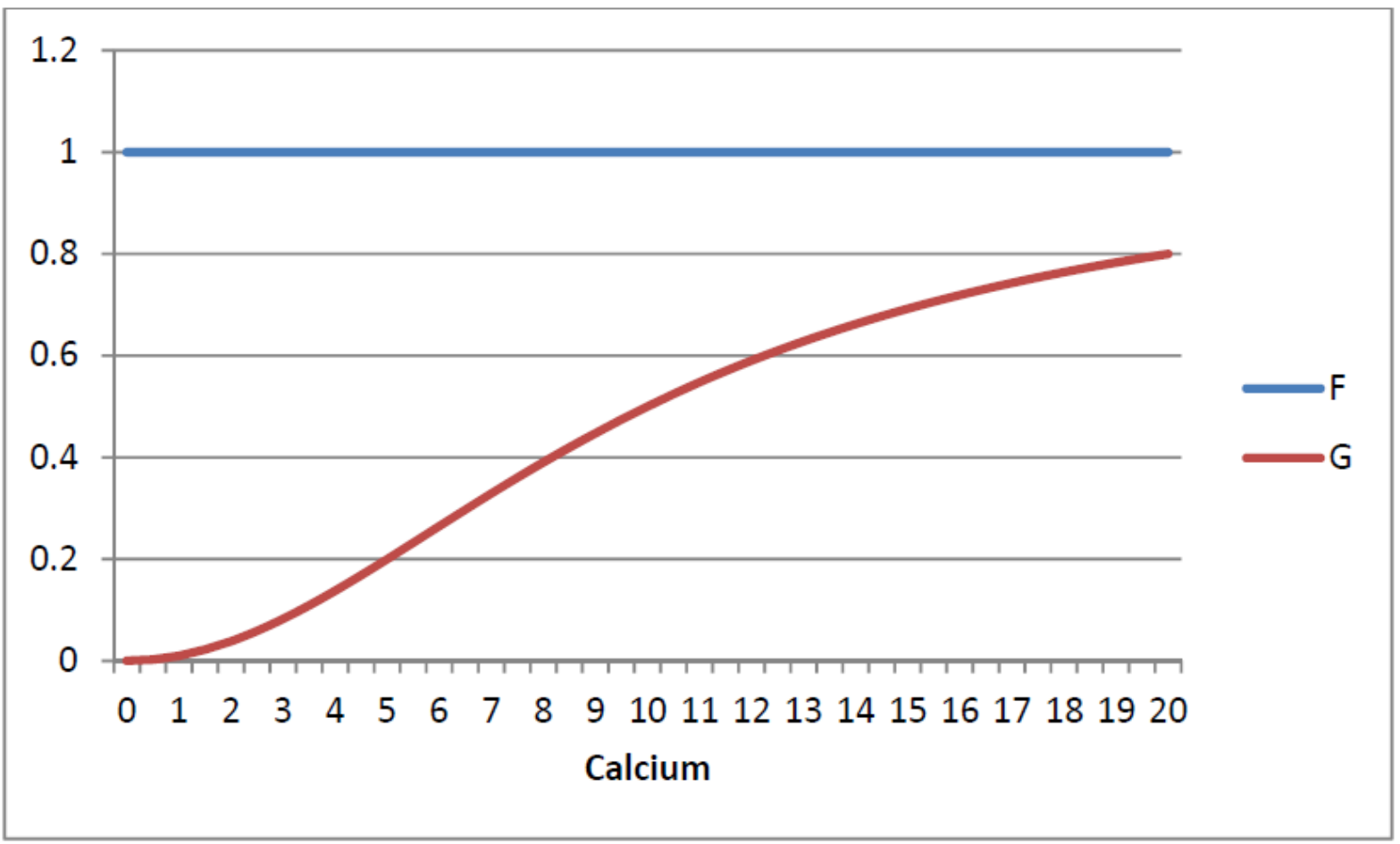

FIGURE 8. HOW CALCIUM AFFECTS GROWTH KF=0, KG=10

The morphological differences between these cells can be created by the simulation by controlling the rate at which MAP-2 blends between its bound state and its phosphorylated state. Elongation is promoted by a prevalence free tubulin and bound MAP-2. The probability of branching is determined by the relative amount of phosphorylated MAP-2. The scale factors determining the rate of conversion from bound to phosphorylated $(\mathrm{F})$ and phosphorylated to bound (G) MAP-2, as particular functions of calcium are shown in Figure 8. In this example, F is constant, while $\mathrm{G}$ increases with calcium. Depending on the values of the rate constants, $\mathrm{c}_{3}$ and $\mathrm{c}_{4}$, the rate of phosphorylation, $\mathrm{c}_{3} \mathrm{~F}$, may be overtaken by the rate of dephosphorylation, $\mathrm{c}_{4} \mathrm{G}$, resulting in a switch from a high branching probability and low elongation state, to a lower branching and higher elongation state. Characteristic switching curves, the steepness of which are determined by the ratio of $c_{3}$ to $c_{4}$, are shown in Figure 9. Having a much larger $c 3$ than $c 4$ will increase the amount of change when moving from phosphorylated to bound MAP-2 for example. So while calcium does not have a direct influence on the branching potential, it does 
have an indirect influence via its altering of MAP-2 states such that figure 9 can be produced correlating the two seemingly independent elements.

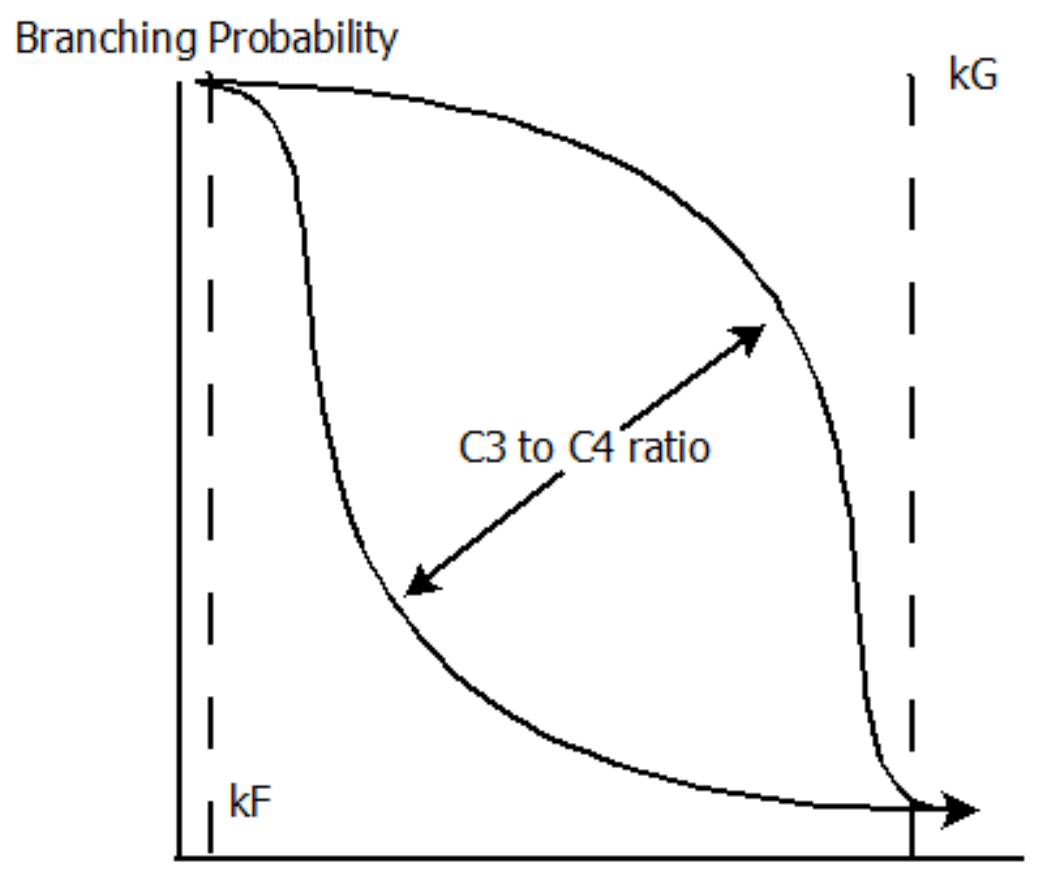

Calcium

FIGURE 9. SWITCHING BETWEEN PHASE STATES (LEFT) HIGH C3/C4 RATIO, (RIGHT) LOW C3/C4 RATIO.

In order to produce a tree with long terminal segments, the initial elongation rate needs to be inhibited and the branching rate increased. This occurs by having a very low $\mathrm{kF}$ and a high $\mathrm{kG}$, for this tree example a $\mathrm{kF}$ of 0 and a $\mathrm{kG}$ of 10 provides this high branching rate. As the amount of calcium approaches 10, the two rates equalise as both F and G approach 1. Once the two rates equalise, the change constants c3 and c4 become the important characteristics. If c3 and c4 are level, the amounts of change between the MAP-2 P and MAP-2 B states would remain fairly static, however with a c3 of 0.1 and a c4 of 1 , there is still a larger shift towards the bound state, and this therefore inhibits the amount of branching potential and promotes the elongation factors. Table 4 has the results under the simulation data column, and shows that the average length of the terminal segments are roughly six times longer than the average length of the intermediate segments. 


\subsection{Unbranched Trees}

In a certain percentage of runs of the simulation there are no branching events at all. These can be seen when the order is zero, there are no intermediate segments and there is a single long terminal segment. This is to be expected in a system where branching is a random event even when there is a high possibility of a branching event occurring. The longer the terminal segment, it appears the less likely it is to have a branching event. Once a tree elongates to a particular length, it is unlikely to ever branch without outside stimulus. This is due to the effects of calcium acting on the rate of change of MAP-2 between the bound and phosphorylated states as outlined above. The time that branching will occur is very early in the lifetime of the simulation given the conditions as discussed, as calcium builds continuously and over reaching a certain level, effectively shuts off branching and promotes elongation.

These were initially discarded as outliers, but they show an important element of the data sets. These essentially become elongation models as discussed previously. These therefore show the differences between the model during branching and what the result would have been using an elongation model under the same circumstances and constants. These tend to skew the statistics of the runs where branching does occur, and while the unbranched trees will continue to be shown in the data sets, they are not considered in the analysis or the statistics. It is very likely that similar trees are observed during experimental research and are discarded for having little value towards the purpose of the research. There would be an interesting correlation to be made if the statistics on the frequency of unbranched trees developed in vitro were released along with other statistical information about morphology. The amount of trees that fail to branch versus those that do in the simulation could then be measured against the observable data produced by researchers. 


\subsection{Results}

The set of parameters which enabled this data set to be created and which forms the base of all the other data sets (treat as defaults, and where there are differences in parameter values, these will be noted), is in table 3 . As discussed, the c4 parameter is much higher than $\mathrm{c} 3$, and $\mathrm{kG}$ is much higher than $\mathrm{kF}$ in order to produce the swing from branching to elongation that's required. The parameters are all arbitrary units, diffusion has been normalised to 1, and the values of the other parameters in the simulation have been worked relative to this to allow proper tree behaviour.

The parameters were initially created by a genetic algorithm which if left to complete would have taken almost 48 months at the rate it was processing, so once there was a set of parameters which were producing trees which were close to the desired results, these were then tweaked by hand to the values listed in table 3. These parameters produce the default model which has long terminal branches.

The decay rates and disassembly rate seems very small, to the point where they appear to have little to none effect on the model at all, but these are multiplicative values and do indeed have a tempering effect on the rate of growth. If the Tubulin decay rate is set to zero for example, there is a massive initial amount of growth where the tree has an extremely long and implausible root intermediate segment.

\begin{tabular}{|l|l|l|l|}
\hline Parameter & Value & Parameter & Value \\
\hline Compartment Length & 2 & Diffusion rate (D) & 1 \\
\hline c1 & 0.8 & Active Transport rate ( $)$ & 0.03 \\
\hline c2 & 0.1 & Assembly rate $(\varepsilon)$ & 0.1 \\
\hline c3 & 0.1 & Disassembly rate $(\psi)$ & 0.00005 \\
\hline c4 & 1 & Calcium Production (I) & 0.3 \\
\hline Conversion constant F $\left(\mathrm{k}^{\mathrm{F}}\right)$ & 0 & Tubulin Production (P) & 1 \\
\hline
\end{tabular}




\begin{tabular}{|l|l|l|l|}
\hline Conversion constant $\mathrm{G}\left(\mathrm{k}^{\mathrm{G}}\right)$ & 10 & MAP-2 U Production $(\mathrm{S})$ & 1 \\
\hline Conversion power constant $\mathrm{F}\left(\mathrm{P}^{\mathrm{F}}\right)$ & 2 & Calcium Decay $\left(\delta_{c}\right)$ & 0.0001 \\
\hline Conversion power constant $\mathrm{G}\left(\mathrm{P}^{\mathrm{G}}\right)$ & 2 & Tubulin Decay $\left(\delta_{t}\right)$ & 0.0001 \\
\hline Branching constant $\left(\mathrm{k}^{\mathrm{B}}\right)$ & 0.02 & MAP-2 U Decay $\left(\delta_{u}\right)$ & 0.0001 \\
\hline Elongation constant $\left(\mathrm{k}^{\mathrm{E}}\right)$ & 3 & MAP-2 B Decay $\left(\delta_{b}\right)$ & 0.0001 \\
\hline Time step $(\mathrm{dt})$ & & & 0.0001 \\
\hline
\end{tabular}

Table 3. The parameter set for producing trees with long terminals.

This parameter set produced the trees shown below. The concentrations of the underlying chemicals over time are shown in figures 10-13.

The calcium concentration in the initial terminal segment is shown in figure 10. It shows that the calcium increases steadily over time, which ties back to figures 8 and 9. This means a short branching period at the start of the model and then a long elongation period.

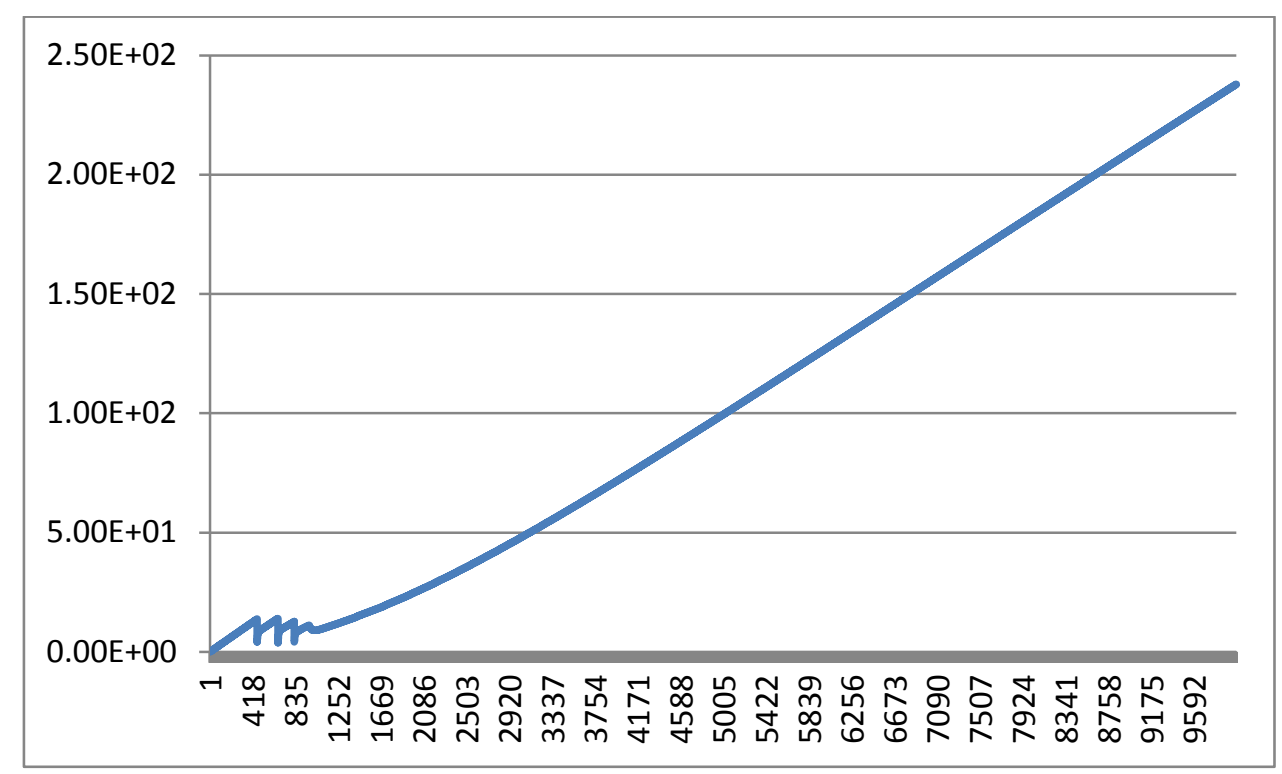

FIGURE 10. CALCIUM IN THE INITIAL TERMINAL SEGMENT OVER TIME. 
The three states of MAP-2 in relation to each other, for the long terminal model are shown in figure 11. It shows that the amount of Bound and phosphorylated MAP-2 is the most defining factor in how the tree grows. The ratio between MAP-2(BP) and MAP-2(B) is initially high, and falls off sharply as branching events occur, until the ratio is sufficiently low enough to inhibit branching and promote elongation.

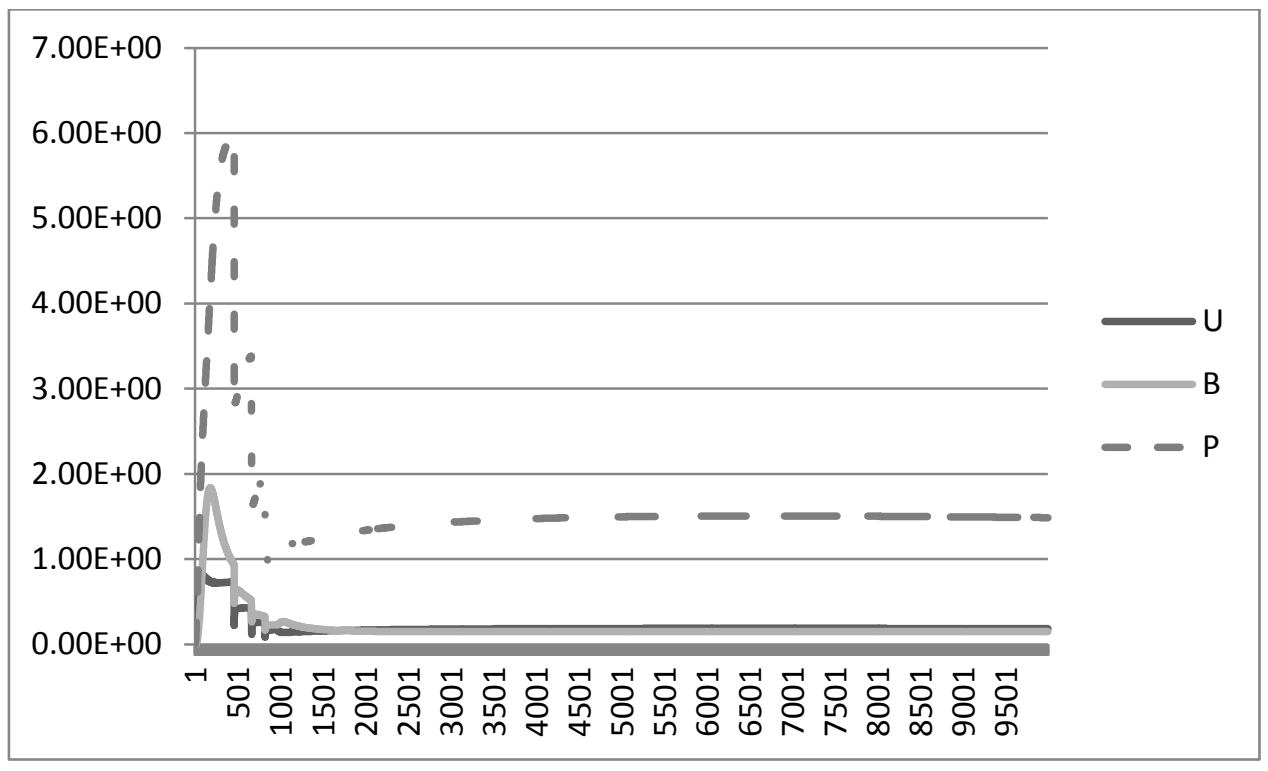

FIGURE 11. MAP-2 STATES IN RELATION TO EACH OTHER IN THE INITIAL TERMINAL SEGMENT OVER TIME.

The amount of free tubulin in the initial terminal segment is shown in figure 12. It shows that the amount of free tubulin in the segment is high, as there is an initial period of high growth of the trunk of the dendrite. As more branching events happen, the amount of free tubulin is spread out over all the terminals, until a steady state is achieved, where the amount of tubulin reaching the terminal segments is enough to promote sufficient growth. 


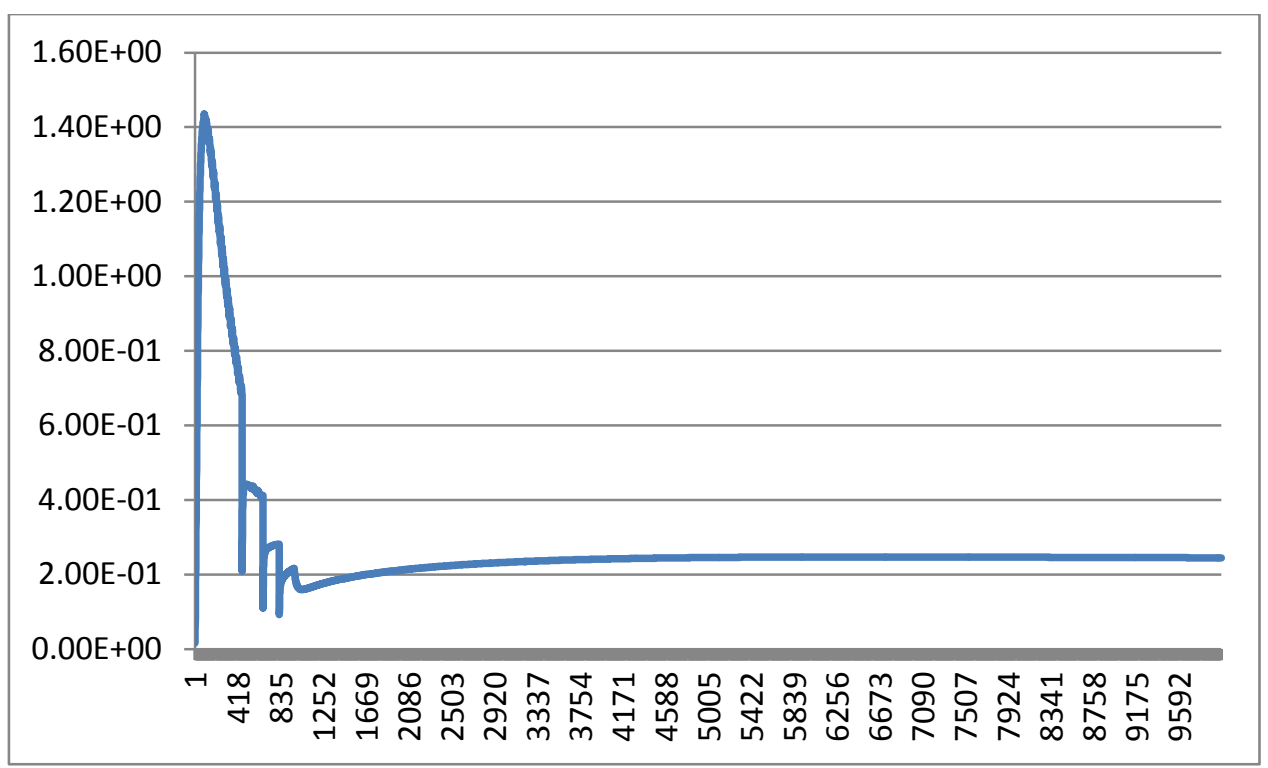

FIGURE 12. FREE TUBULIN IN THE INITIAL TERMINAL SEGMENT OVER TIME.

The probability of a branching event occurring and the amount of elongation in the initial terminal segment over time is shown in figure 13. Figure 13(left) shows that the branching probability is initially high, producing several branches, and if compared to the levels of MAP2(BP), drops off at roughly the same rate until the ratio of MAP-2(BP) to MAP-2(B) is low enough to prevent branching from occurring. Figure 13(right) shows that the amount of elongation in the terminal segment is initially high as there is no competition for resources and so it is consuming them all in an initial burst of growth. Once there are several branching events, the growth levels drop off to a steady state of elongation.
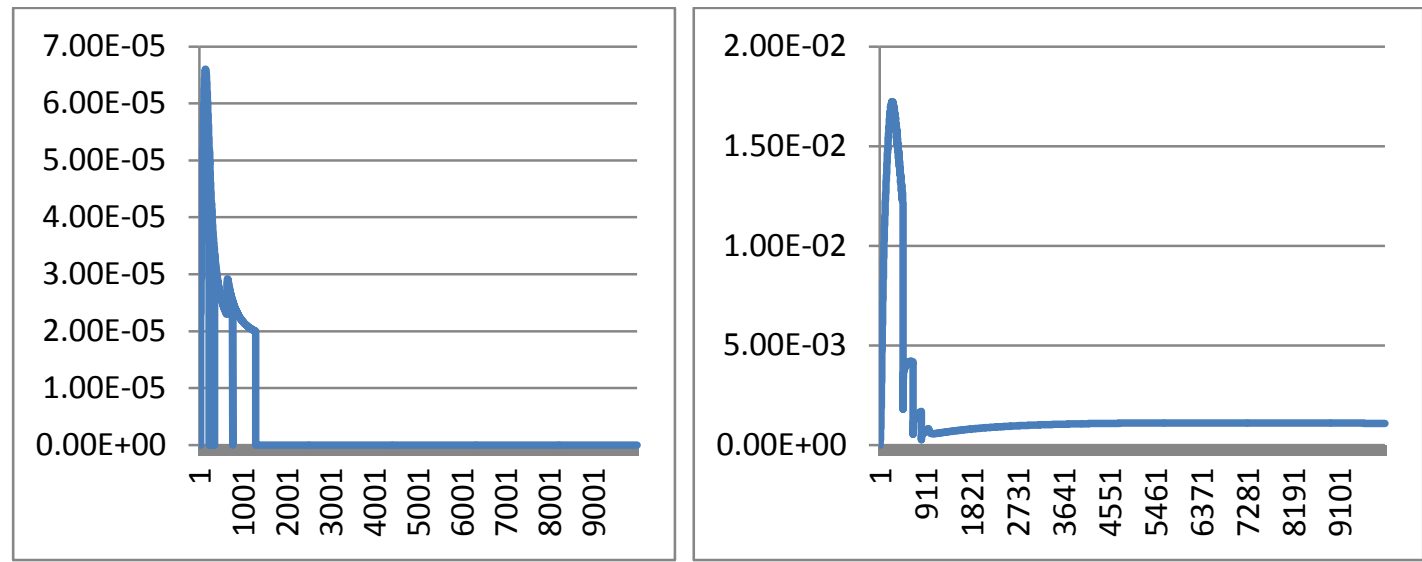

FIGURE 13. (LEFT) THE PROBABILITY OF BRANCHING IN THE INITIAL TERMINAL SEGMENT OVER TIME. (RIGHT) THE AMOUNT OF ELONGATION IN THE INITIAL TERMINAL SEGMENT OVER TIME. 
The simulation can produce many varied results based on the values entered for the constants. The model was set up to allow the probability of branching events to decrease over time as outlined above. The data set had the unbranched trees removed before the statistics were produced, and the results are shown in table 3. The simulation was set to perform a thousand runs, however the number in the final data set is lower once the unbranched trees were removed.

The simulation was set to run for one thousand discrete time units, and with a $d t$ of 0.01 to maintain integral accuracy within the system, this resulted in a total amount of one hundred thousand time bins for the simulation to iterate over. The time units and $d t$ were not linked to "real time" in any way. The simulation was to run for the full length of the time, regardless of whether the simulation had reached a steady state or was still developing at the end of the allotted time. A steady state is not completely possible in a system which relies on random chance for its branching events, as a single branching event could disrupt the state and start the alteration of the morphology. To allow for this event to occur, all the simulations ran till the conclusion of the allotted time.

\begin{tabular}{|l|l|l|}
\hline Morphological variable & Biological data & Simulation data \\
\hline Terminal Length & $117+/-33$ & $126.9+/-30.7$ \\
\hline Intermediate Length & $15.4+/-13.4$ & $22+/-15.7$ \\
\hline Path Length & $156+/-29$ & $177.7+/-6.7$ \\
\hline Order & - & $1.5+/-0.86$ \\
\hline
\end{tabular}

Table 4. Rat basal pyramidal cell dendrite data taken from Larkman(1991), compared to the data resulting from the simulation of the model.

As can be seen, the simulation data and the biological data are closely related, with the average values and the standard deviation of the simulation closely relating to the values experimentally 
observed. The mean is slightly on the long side but well within a reasonable margin of error, the standard deviation is closer to the biological model. Figure 14 shows the histograms of the data set that was used to generate these statistics.
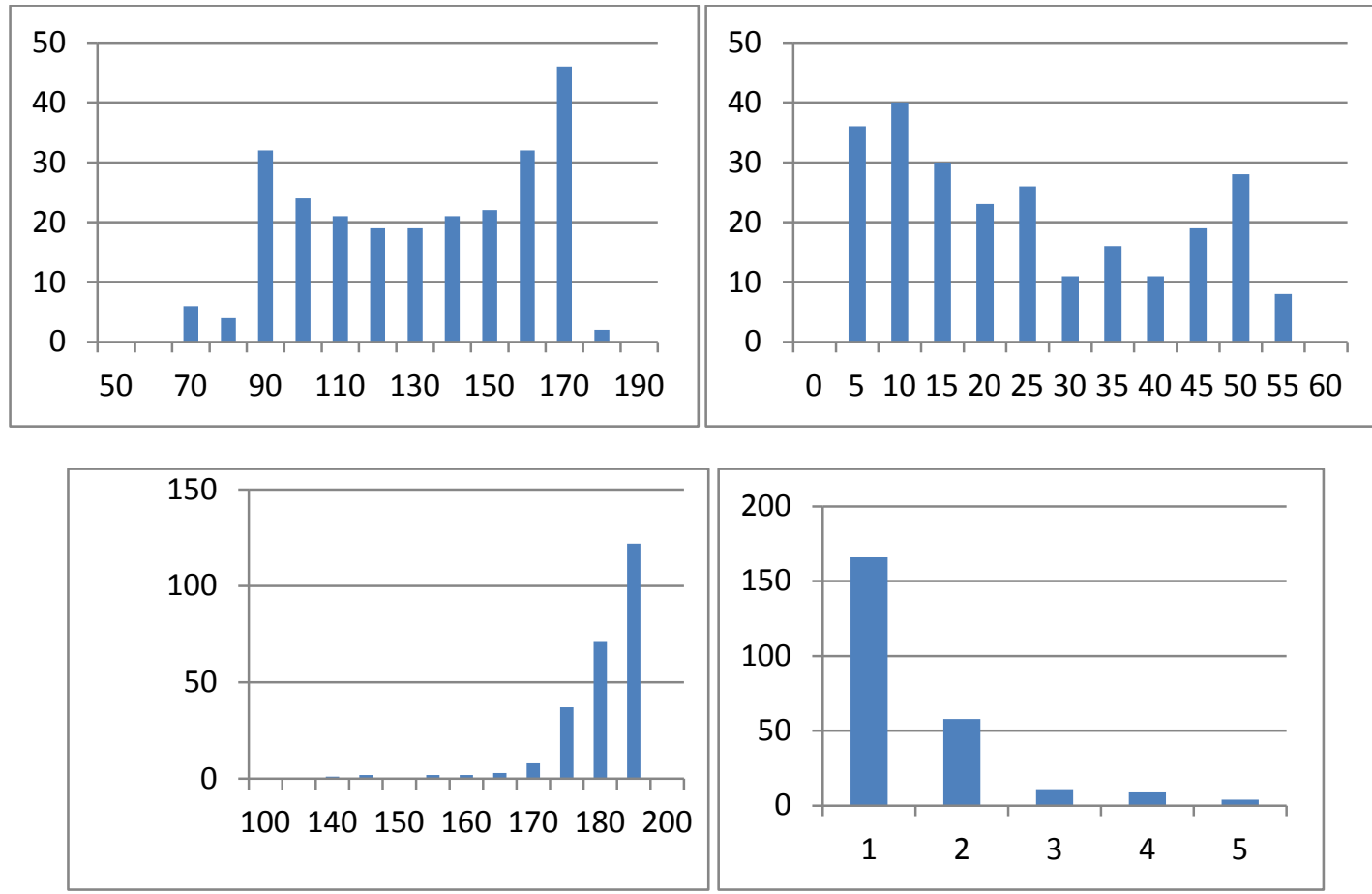

FIGURE 14. (TOP LEFT) THE HISTOGRAM FOR TERMINAL LENGTH, (TOP RIGHT) THE HISTOGRAM FOR INTERMEDIATE LENGTH, (BOTTOM LEFT) THE HISTOGRAM FOR PATH LENGTHS, (BOTTOM RIGHT) THE HISTOGRAM FOR THE ORDER.

As can be seen, the path lengths are consistent with a small standard deviation as shown in table

4. This is likely due to the time spent in the elongation phase of the development of the cell is very similar in all cases and path length ignores many of the effects of branching by tracing the path from the some to the tips of the branches. The order of the simulation tends to be quite low. Given exact simulation times, similar branching degrees, and similar calcium concentrations, the differentiating point here will be the timing of the initial branching event: the later it comes, the shorter the path. The reason this is an important differentiating point is that an early initial branch allows for more branching to occur during the branching period, as each branching event increases the pool of terminals to which branching events can occur, and the more branches, the longer the overall path length. 
The intermediate lengths are clustered around the shorter end of the scale around the same values as the mean in the biological data set, however the length of these segments is more variable and a broader scale of values pushes the mean higher than the biological set. If the length of these segments was slightly scaled back, perhaps with one or two more branches on average, the values would be very close. The terminal lengths histogram is fairly flat, clustered in a range around the mean and have a significant number around the mean of the biological model with few lying outside of the acceptable range. Those that are, are again on the long side, which is a situation which would be brought back into check with one or two more branching event occurring. Further work on tuning the parameters to more closely align the model with the statistical results would be necessary.

\subsubsection{Producing different trees}

The results in figure 15 show that the model can produce several different tree topologies with minimal changes to the parameters. The initial tree type displays a relatively uniform segment length throughout the tree (Fig.15(left)). The other tree types have long intermediate segments with short terminal segments (Fig. 15(center)) or long terminal segments (Fig. 15(right)). These tree types are consistent with similar topologies produced by Hely’s MAP-2 model (Hely et al 2001) and statistical information from real neuronal trees (van Pelt and Uylings 1999).
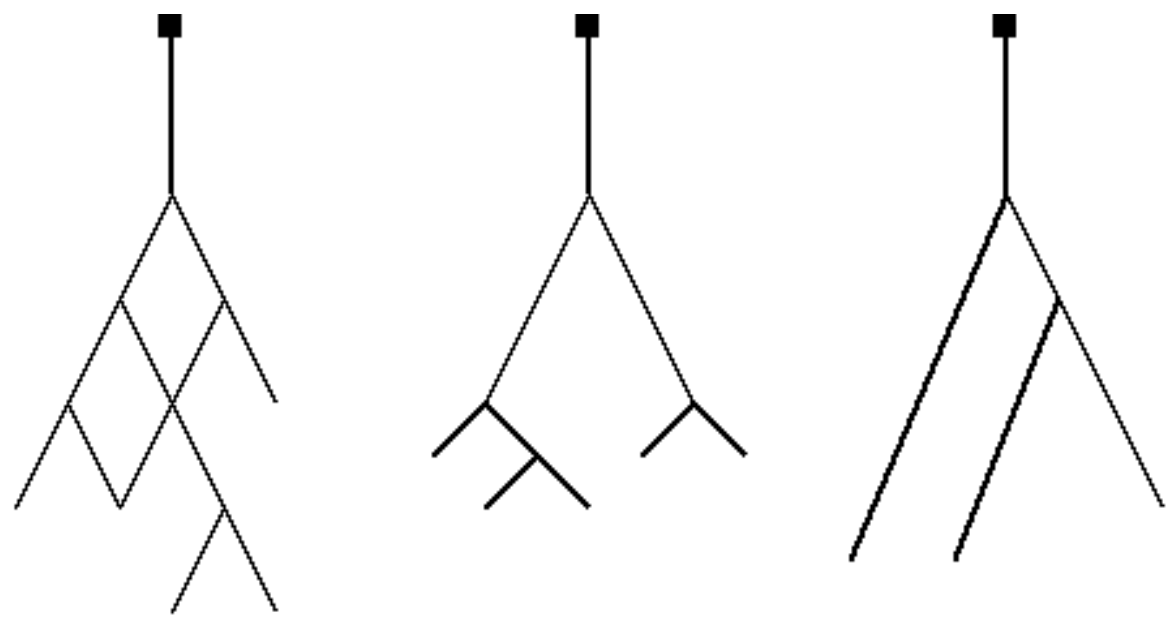

FIGURE 15. (LEFT) SIMILAR LENGTH SEGMENTS, (CENTRE) SHORTER TERMINAL SEGMENTS, (RIGHT) LONGER TERMINAL SEGMENTS (THE DEFAULT MODEL) 
Figures 16 and 17 show the differences in segment lengths and order, along with the changes in parameter values to generate those trees. The histograms display data from 1000 simulated trees for the similar length, and the shorter terminal topologies are shown in figure 16, the histograms for the longer terminals were shown in figure 14. Again, any unbranched trees have been removed from the data sets before any statistics were generated.
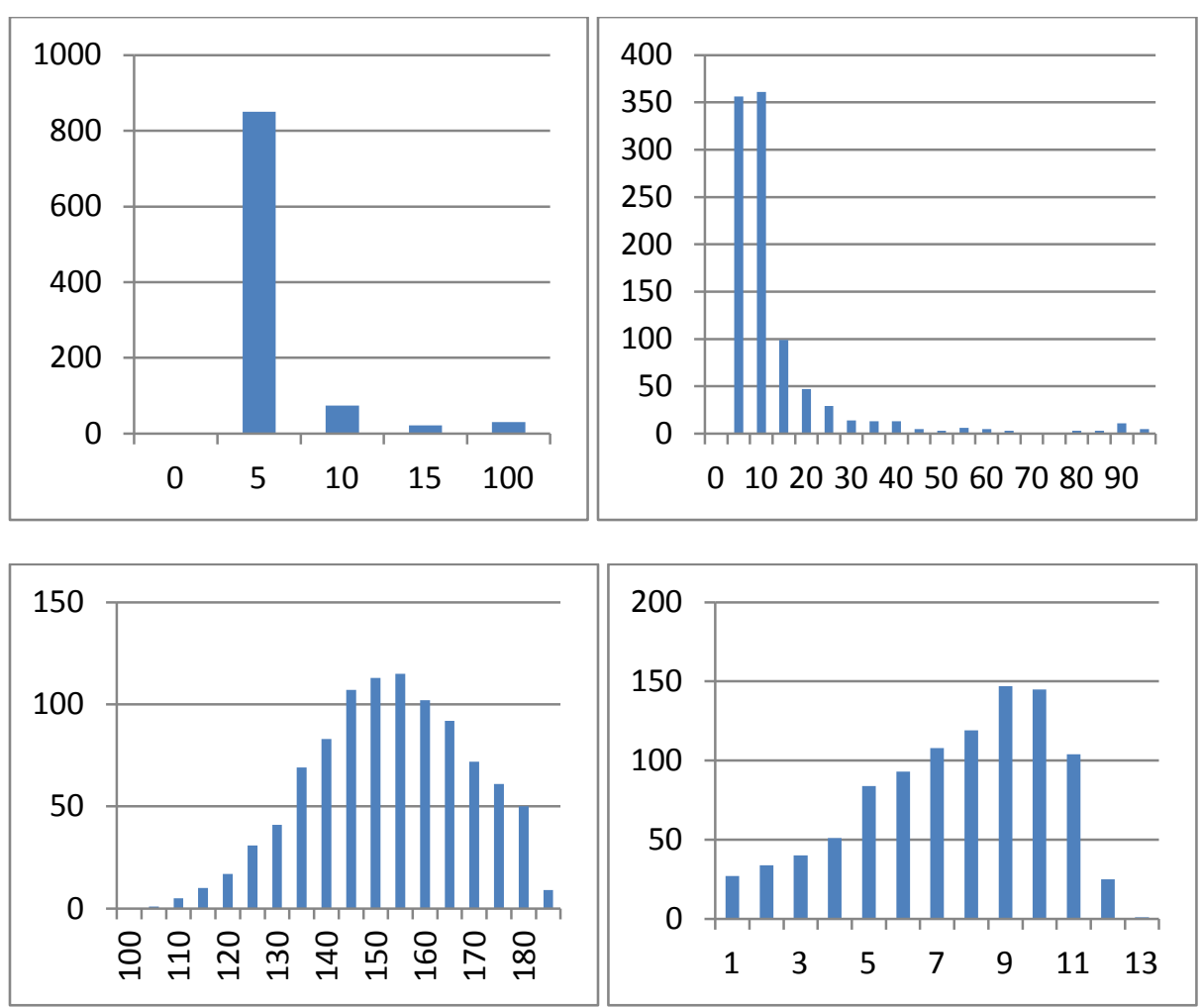

FIGURE 16. (TOP LEFT)TERMINAL, (TOP RIGHT)INTERMEDIATE LENGTHS, (BOTTOM LEFT) PATH LENGTH, (BOTTOM RIGHT) ORDER FOR SIMILAR LENGTH TREE

The difference in lengths is significant. The tree produced in figure 15(left) and shown in figure 16 have an intermediate to terminal ratio of approximately one to six and the tree produced in figure 15(center) and shown in figure 17 have an intermediate to terminal ratio of six to one. 


\begin{tabular}{|l|l|}
\hline Morphological variable & Simulation data \\
\hline Average Terminal Length & $3.22+/-6.19$ \\
\hline Average Intermediate Length & $11.16+/-6.19$ \\
\hline Path Length & $150.19+/-15.87$ \\
\hline Order & $7.5+/-2.76$ \\
\hline
\end{tabular}

Table 4. Statistical data for the Similar length tree.
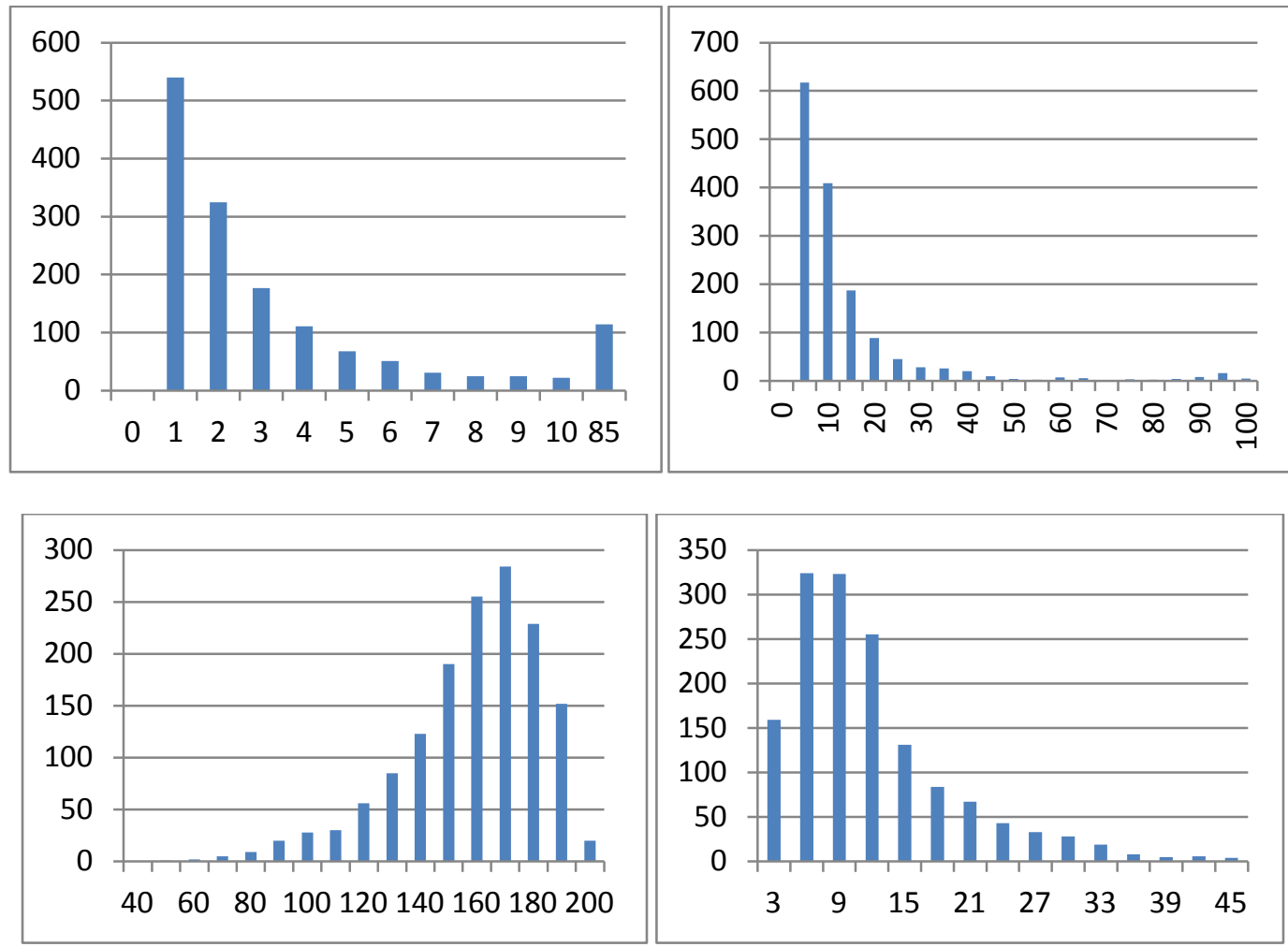

FIGURE 17. (TOP LEFT) TERMINAL (TOP RIGHT) INTERMEDIATE (BOTTOM LEFT) PATH LENGTHS AND (BOTTOM RIGHT) ORDER FOR SHORT TREE

The differences between the three topologies have been generated by changing the rate constants that dictate how much MAP-2 converts to and from its unbound, bound and phosphorylated states. By increasing the rate at which bound MAP-2 is converted into phosphorylated MAP-2 (c3) and reducing the rate at which phosphorylated MAP-2 is turned back into bound MAP-2 (c4), the average amount of phosphorylated MAP-2 increases. This 
raises the probability of branching and results in longer intermediate segments and shorter terminal segments, as can be seen in figures 15(centre) and 17. Decreasing the rate at which bound MAP-2 is converted into phosphorylated MAP-2 (c3) and increasing the rate at which phosphorylated MAP-2 is turned back into bound MAP-2 (c4), reduces the amount of phosphorylated MAP-2 and branching decreases. This promotes elongation and results in shorter intermediate segments and longer terminal segments, as can be seen in figure 15 (right) and figure 14. The branching point in the simulation was also moved in order to produce longer intermediate segments. A much higher amount of calcium was required to switch from elongation to branching, and so $\mathrm{kF}$ was set to very low and $\mathrm{kG}$ was set to a much higher value.

\begin{tabular}{|l|l|}
\hline Morphological variable & Simulation data \\
\hline Average Terminal Length & $3.53+/-6.18$ \\
\hline Average Intermediate Length & $11.35+/-15.85$ \\
\hline Path Length & $153.27+/-24.72$ \\
\hline Order & $10.76+/-7.56$ \\
\hline
\end{tabular}

Table 5. Statistical data for short terminal tree.

The reason the promotion of elongation results in longer terminal segments is similar to the reasons for unbranched trees. As the terminal segments grow in length the chances of them branching decrease resulting in the morphology shown. Likewise, inhibiting elongation somewhat means greater concentrations of phosphorylated MAP-2 build up and allows branching events to become more likely. 
The rate constants for the three trees above are shown in table 6 .

\begin{tabular}{|l|l|l|l|}
\hline Parameter & Long & Equal & Short \\
& Terminal & Terminal & Terminal \\
& Lengths & Lengths & Lengths \\
\hline Conversion constant kF & 0 & 0 & 10 \\
\hline Conversion constant kG & 10 & 10 & 0 \\
\hline c1 & 0.8 & 0.8 & 0.8 \\
\hline c2 & 0.1 & 0.1 & 0.1 \\
\hline c3 & 0.1 & 0.2 & 0.2 \\
\hline c4 & 1 & 1 & 0.85 \\
\hline
\end{tabular}

Table 6. Rate constants for the three types of neuronal development.

By increasing c3 we are inhibiting growth and promoting branching heavily. By increasing c4 we are promoting elongation while inhibiting branching heavily. The rate constants are the most engaging way to alter the morphology of the tree. They directly control the interactions between the states of MAP-2 and therefore have the largest effect on the tree. They are also the least natural means of altering the tree. Altering the way in which calcium affects the conversions, or controlling the rate in which chemicals are added to the tree are closer to the effects seen in nature. However an argument could be made that the rate constants correlate with the different types of neuronal types seen in biology and so that these rate constants are actually part of the biological makeup of the cells, that is to say that different cells under identical conditions will definitely grow differently due to their internal make up.

The calcium in the system is forever increasing, as it starts of low and never truly reaches a steady state. This is due to each compartment having influx of calcium, and the diffusion of older compartments which contain larger concentrations to newer compartments with lower concentrations. This provides us with a convenient steady, and reliable factor which has a direct 
impact on the morphology through the rate conversion constants. The rate change constants $\mathrm{kF}$ and $\mathrm{kG}$ play a vital role in producing these different morphologies. They are required to be set to the correct amount of calcium in the system so that the branching activation point switches appropriately, whether that is from branching over to elongation, or from elongation to branching. Lowering or raising these values has the ultimate effect of shifting this change point in time due to the roughly linear nature of the calcium increases being equatable to time.

\subsubsection{Changing the calcium conversion rate ( $\mathrm{kG}$ and $\mathrm{kF})$}

The default values for the parameters produce trees with relatively short intermediate segments and longer terminal segments. By changing the calcium rate conversion points governed by the conversion rate constants ( $\mathrm{kF}$ and $\mathrm{kG}$ ), we are able to shift the switching point between a branching state and a elongation state sooner or later in time. The default tree has a very low value for $\mathrm{kF}$ and a relatively low value for $\mathrm{kG}$. This is to ensure that the branching stage of the neurite development lasts a short time at the beginning of its life cycle and that there is a long elongation phase. This is what produces the long terminal segments.

\subsubsection{High kG, low kF}

Table 7 shows the values when we set kG to a much higher value.

\begin{tabular}{|l|l|}
\hline Parameter & Value \\
\hline Conversion constant kF & 0 \\
\hline Conversion constant kG & 30 \\
\hline
\end{tabular}

Table 7. The values for a high $\mathrm{kG}$. 
The histograms in figure 18 show the differences when $\mathrm{kG}$ is set to a higher value.
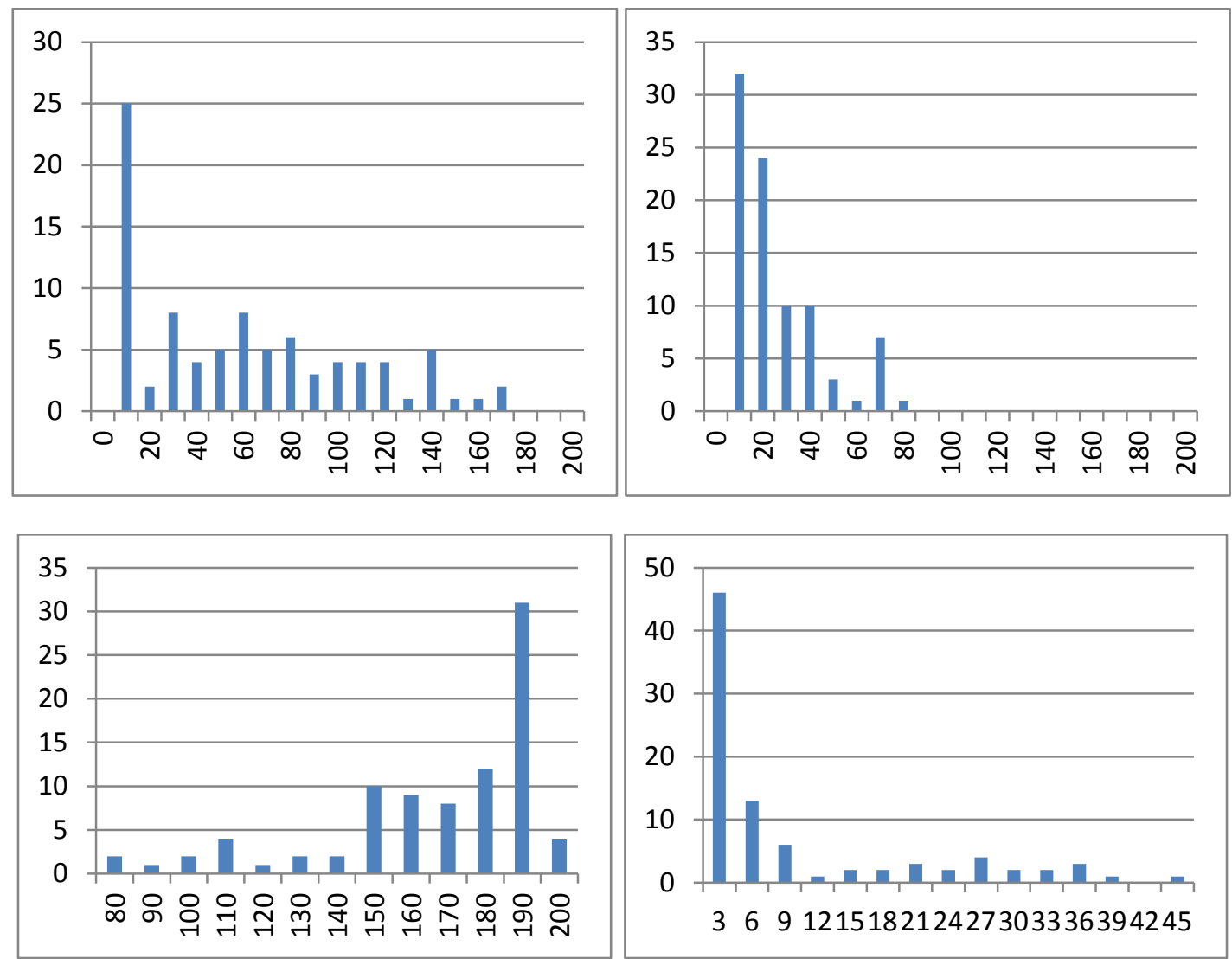

FIGURE 18. (TOP LEFT)TERMINAL (TOP RIGHT)INTERMEDIATE (BOTTOM LEFT) PATH LENGTHS AND (BOTTOM RIGHT) ORDER FOR HIGHER KG

There are longer intermediate segments and shorter terminal segments due to the longer amount of time spent in the branching phase of the neurite outgrowth and less time growing the terminals which exist when the phase change from branching to elongation occurs. The high c4 still inhibits the amount of phosphorylated MAP-2 in the system and so allows the intermediates to grow further before branching rather than allowing a lot of branching to occur in a short space of time, which would lead to drastically smaller average intermediate segments. Setting the value of kG higher would continue this process further but would start to have a larger amount of branching. Once there is a critical mass of branching events in a short space of time the calcium levels drop very low (due to the need to conserve concentrations when creating new compartments) which encourages more branching, which lowers the calcium 
levels, until the system is doing little else other than branching and the switching point between branching and elongation never occurs.

\begin{tabular}{|l|l|}
\hline Morphological variable & Simulation data \\
\hline Average Terminal Length & $54.2+/-47.98$ \\
\hline Average Intermediate Length & $20.13+/-19.61$ \\
\hline Path Length & $162+/-29.09$ \\
\hline Order & $8.92+/-11.13$ \\
\hline
\end{tabular}

Table 8. Statistical data for the high kG tree.

\subsubsection{Equal kG and kF}

Table 9 shows the values when we set $\mathrm{kG}$ and $\mathrm{kF}$ to equal values.

\begin{tabular}{|l|l|}
\hline Parameter & Value \\
\hline Conversion constant kF & 10 \\
\hline Conversion constant kG & 10 \\
\hline
\end{tabular}

Table 9. The values for equal $\mathrm{kF}$ and $\mathrm{kG}$.

These parameters do not produce any useful trees at all, every single tree is an unbranched one, with a single long terminal segment. This is due to the fact there is no branching phase in the model when he conversion constants are the same and the c4 constant heavily outweighs the c3 constant. The $\mathrm{F}$ and $\mathrm{G}$ equations will both always be equal, neutralising the effect calcium has upon the state of the neurite. This leaves only the c constants to impose order on how the system grows. Therefore, there is only a constant elongation phase producing no branches. 


\subsubsection{Low kG, high kF}

Table 10 shows the values when we set the $\mathrm{kG}$ to a low value and $\mathrm{kF}$ to a high value.

\begin{tabular}{|l|l|}
\hline Parameter & Value \\
\hline Conversion constant kF & 30 \\
\hline Conversion constant kG & 0 \\
\hline
\end{tabular}

Table 10. The values for high $\mathrm{kF}$ and low kG.

These parameters produce no useful trees, every single tree is an unbranched one, with no branches and a single long terminal segment. This is due to the fact there is no branching phase in the model when the conversion constants are the same and the c4 constant heavily outweighs the $\mathrm{c} 3$ constant. The $\mathrm{G}$ equation will be much greater than the $\mathrm{F}$ equation until there is a significant amount of calcium in the system and then the two will be in relative parity. Up to this point the system is in an elongation state, and once the parity is reached, the c constants play a bigger role. Therefore there is only a constant elongation phase after this point producing no branches.

\subsubsection{Overall impact of changing $\mathrm{kF}$ and $\mathrm{kG}$}

Changing $\mathrm{kF}$ to be equal or higher than $\mathrm{kG}$ produces no reasonable results. This is a constant elongation state which produces uninteresting single terminal segment trees. Altering $\mathrm{kG}$ delays or hastens the time between the branching state and the elongation state producing longer intermediate segments than terminal segments when the switching point is delayed.

\subsubsection{Changing the production rate for tubulin $(P)$}

The default value for the amount of tubulin produced ensures there is enough tubulin to be bound to the terminals to allow for a decent amount of growth. By altering the amount of tubulin production the amount of overall elongation in the model should be effected. As the 
equations are based on the relative amounts of concentrations, rather than the concentrations being accurately modelled, increasing $\mathrm{P}$ does not necessarily mean that there is a required increase in other parameters. If the proteins were being modelled fully, and the active transport rate was a set value of how much actual tubulin could be moved from one compartment to the next in a single time step, then overproducing $\mathrm{P}$ would have a lesser effect as it would not be largely transported to the terminal tips. This is lessened but not mitigated by diffusion which will always move a larger amount for one compartment to the next in a single time step as it is accurately based on concentrations rather than discrete values.

\subsubsection{Higher production rate}

Table 11 shows the values when we set the value of $\mathrm{P}$ higher than the default.

\begin{tabular}{|l|l|}
\hline Parameter & Value \\
\hline Tubulin production rate & 1.5 \\
\hline
\end{tabular}

Table 11. The higher production rate of tubulin.

The histograms in figure 19 show the differences when $\mathrm{P}$ is set to a higher value. 

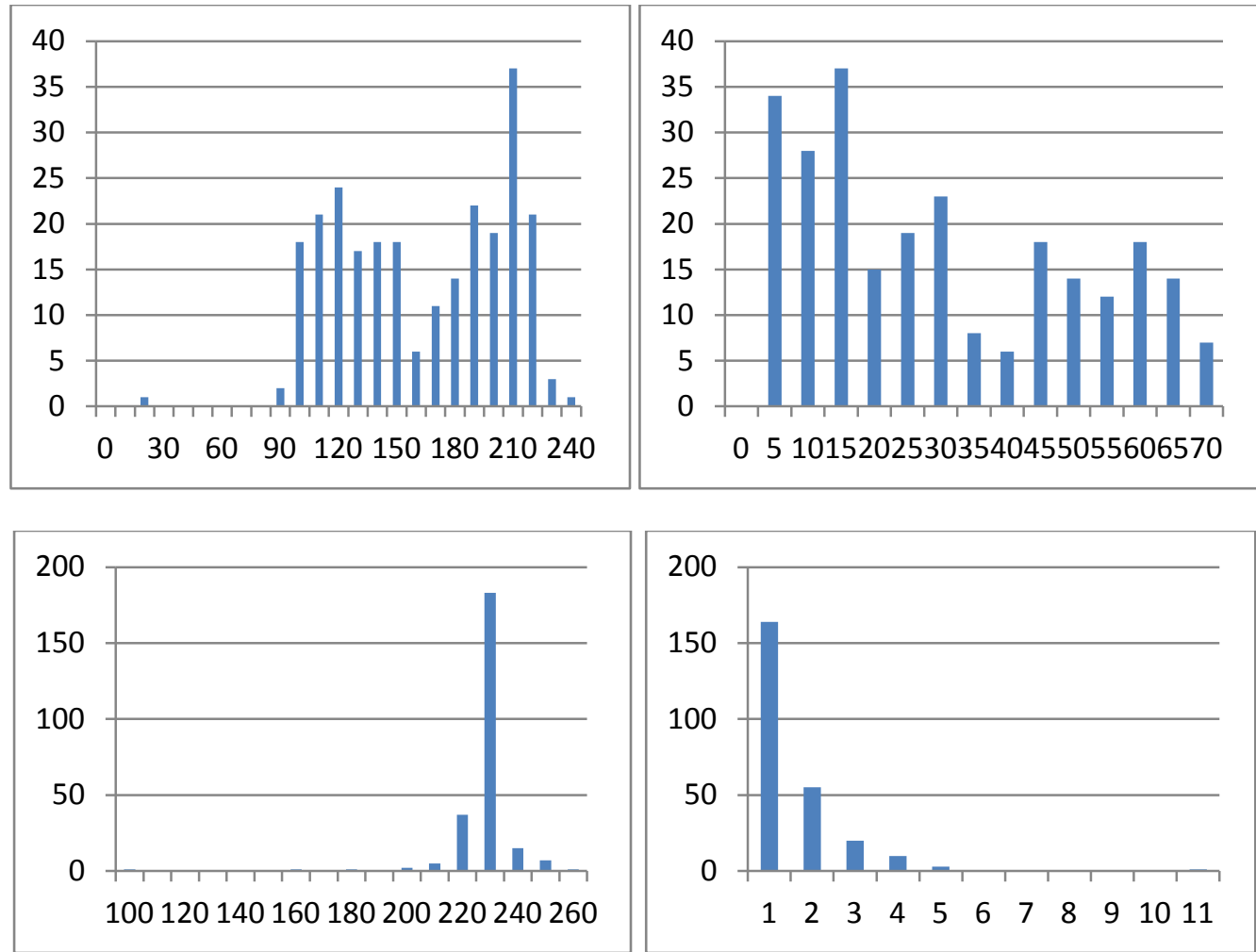

FIGURE 19. (TOP LEFT)TERMINAL (TOP RIGHT)INTERMEDIATE (BOTTOM LEFT) PATH LENGTHS AND (BOTTOM RIGHT) ORDER FOR HIGHER TUBULIN PRODUCTION

This parameter change allows the segments to grow quicker due to a larger amount of tubulin being introduced. Intermediate segments are about twenty five percent longer than those in the default model. The terminal segments are also approximately twenty five percent longer than those in the default model. As expected, the length of the segments is not mitigated by any other factors and has grown accordingly. There is no direct alteration of the branching rates and no direct change in the switch between the branching phase and the elongation phase.

\begin{tabular}{|l|c|}
\hline Morphological variable & Simulation data \\
\hline Average Terminal Length & $158.12+/-42.32$ \\
\hline Average Intermediate Length & $27.49+/-20.29$ \\
\hline Path Length & $223.45+/-12.14$ \\
\hline Order & $1.58+/-1.06$ \\
\hline
\end{tabular}

Table 12. Statistical data for higher tubulin tree. 


\subsubsection{Lower production rate}

Table 13 shows the values when we set the value of $\mathrm{P}$ lower than the default.

\begin{tabular}{|l|l|}
\hline Parameter & Value \\
\hline Tubulin production rate & 0.5 \\
\hline
\end{tabular}

Table 13. The lower production rate for tubulin.

The histograms in figure 20 show the differences when $\mathrm{P}$ is set to a lower value.
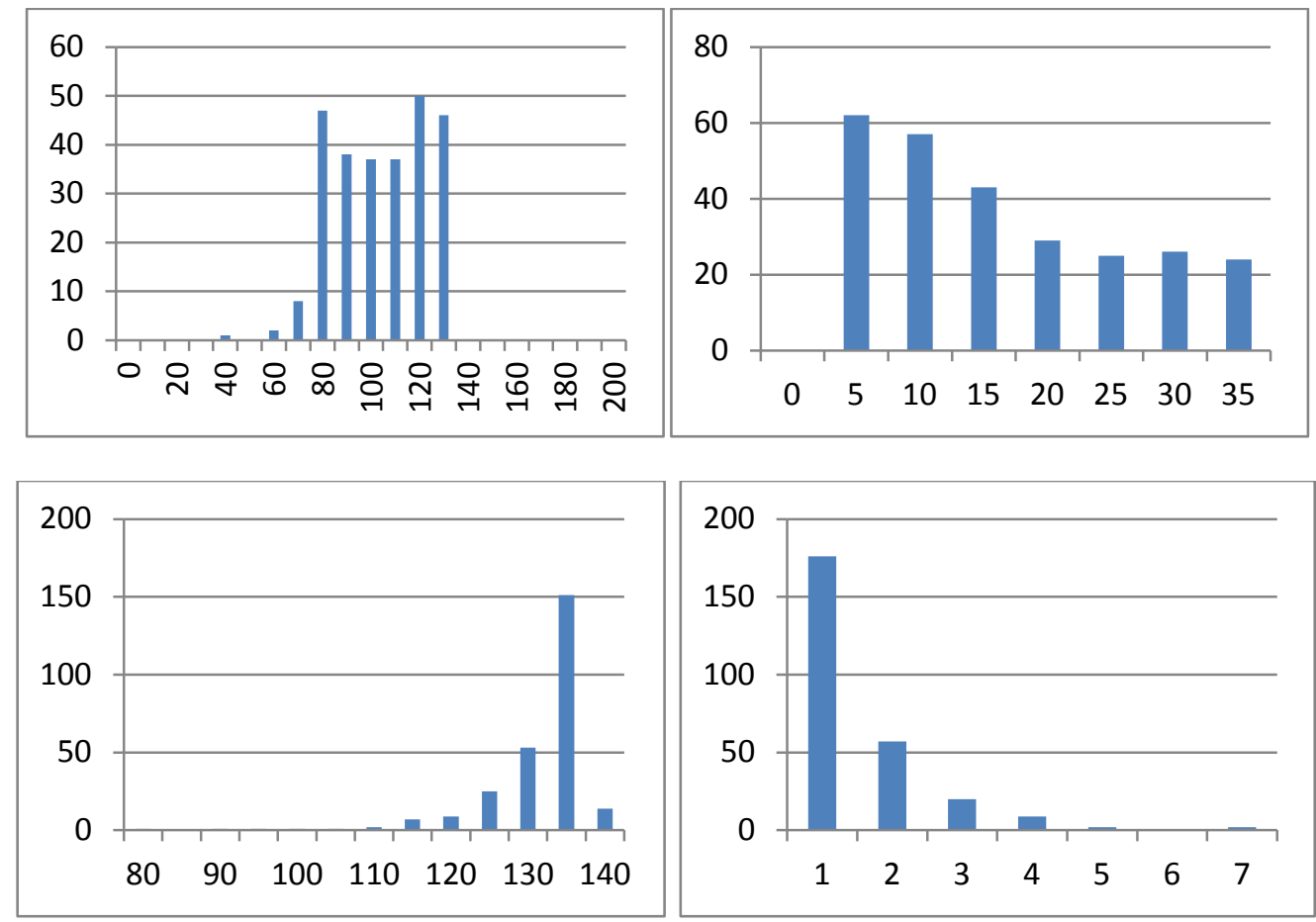

FIGURE 20. (TOP LEFT)TERMINAL (TOP RIGHT)INTERMEDIATE (BOTTOM LEFT) PATH LENGTHS AND (BOTTOM RIGHT) ORDER FOR LOWER TUBULIN PRODUCTION

This parameter change prevents the segments from growing as quickly due to there being a lack of tubulin in order to bind to. Intermediate segments are about twenty five percent shorter than those in the default model. The terminal segments are also approximately twenty five percent shorter than those in the default model. In a model which was handling retraction and microtubule collapse properly, lowering this parameter value would have a harder limit on the 
amount of growth, and would likely lead to fewer branches due to competition starving them of resources and allowing the branches to retract back into the main body of the dendrite.

\begin{tabular}{|l|l|}
\hline Morphological variable & Simulation data \\
\hline Average Terminal Length & $98.56+/-19.03$ \\
\hline Average Intermediate Length & $13.55+/-9.54$ \\
\hline Path Length & $129.32+/-7.79$ \\
\hline Order & $1.54+/-0.95$ \\
\hline
\end{tabular}

Table 14. Statistical data for lower tubulin tree.

\subsubsection{Overall impact of changing $P$}

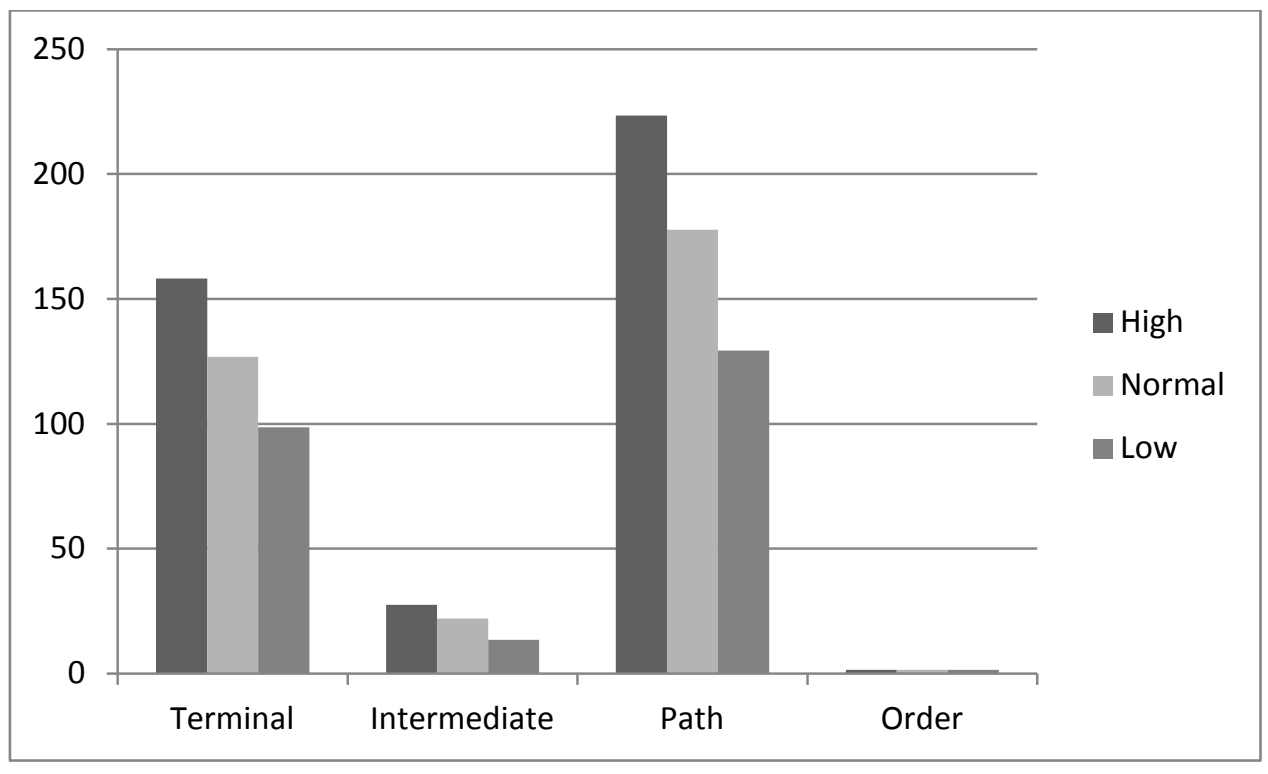

FIGURE 21. OVERALL IMPACT OF CHANGING TUBULIN PRODUCTION

The default value for the production of tubulin ensured there was enough tubulin to produce the long terminal segments in the default mode. By changing the values for the production of tubulin, we either excite the growth of the segments, or inhibit the growth of the segments. Further alterations to the model to allow the accurate modelling of the transport of tubulin and by lowering the amount of free tubulin properly, by not allowing more assembly of tubulin than 
there is free for example, would allow a more plausible modelling of tubulin production and its effects on the simulations.

\subsubsection{Changing the production rate for unbound MAP-2 (S)}

The default value for the amount of unbound MAP-2 ensures that there is enough MAP-2 to allow a proper binding of tubulin to the terminal segments. As the branching factor is related to the ratios of to the states of MAP-2, altering this value will not directly alter the amount of branching in the model. Altering it should however increase or reduce the amount of elongation in the model as the ratios will remain constant but with less bound tubulin with which to elongate. This is due to there being less unbound MAP-2 to convert into the bound state, and the concentration of bound MAP-2 has a direct effect on the amount of tubulin bound into microtubules. The branching rate does not alter as the constants which govern the ratios between the states do not alter.

\subsubsection{Higher production rate}

Table 15 shows the values when we set the value of $\mathrm{S}$ higher than the default.

\begin{tabular}{|l|l|}
\hline Parameter & Value \\
\hline Unbound MAP-2 production rate & 1.5 \\
\hline
\end{tabular}

Table 15. The higher production rate for unbound MAP-2. 
The histograms in figure 22 show the differences when $\mathrm{S}$ is set to a higher value.
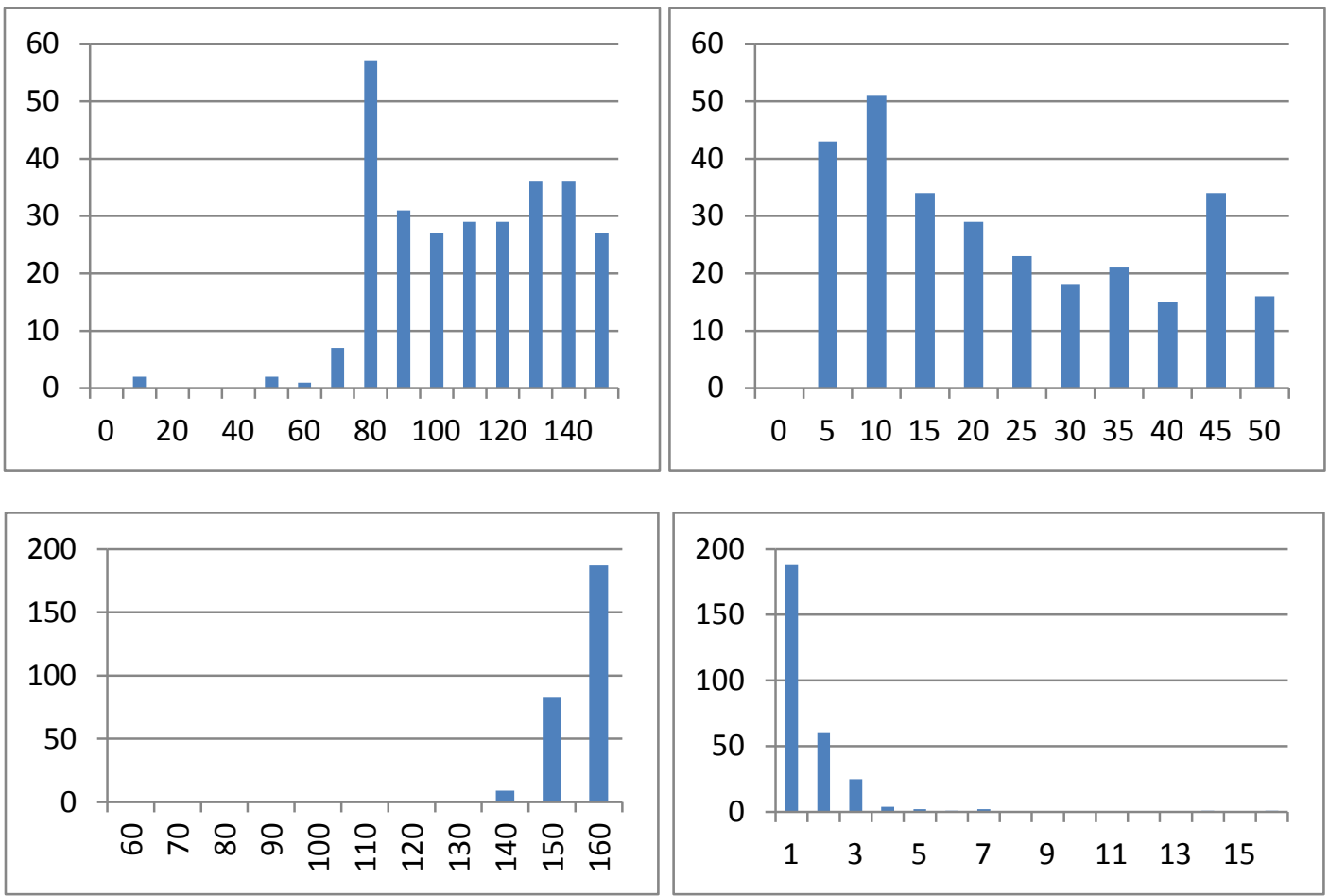

FIGURE 22. (TOP LEFT)TERMINAL (TOP RIGHT)INTERMEDIATE (BOTTOM LEFT) PATH LENGTHS AND (BOTTOM RIGHT) ORDER FOR HIGHER UNBOUND MAP-2 PRODUCTION

Unexpectedly, this parameter change has lowered the length of both the terminal and intermediate segments by approximately ten percent, and has risen the average amount of branches in the simulation by approximately the same amount. The higher branching rate accounts for a lower average length of both segments. This remains within a degree of the data set produced by the default model, and so the initial conclusions are that the change in unbound MAP-2 is not sufficient to drive a significant change in the model. It can also be said that the amount of unbound MAP-2 remains of lesser interest as the branching probabilities are still driven by the ratios of the state change. 


\begin{tabular}{|l|l|}
\hline Morphological variable & Simulation data \\
\hline Average Terminal Length & $104.2+/-26.63$ \\
\hline Average Intermediate Length & $20.53+/-14.43$ \\
\hline Path Length & $151.6+/-11.73$ \\
\hline Order & $1.61+/-1.46$ \\
\hline
\end{tabular}

Table 16. Statistical data for higher unbound MAP-2 tree.

\subsubsection{Lower production rate}

Table 17 shows the values when we set the value of $S$ lower than the default.

\begin{tabular}{|l|l|}
\hline Parameter & Value \\
\hline Unbound MAP-2 production rate & 0.5 \\
\hline
\end{tabular}

Table 17. The lower production rate for unbound MAP-2. 
The histograms in figure 23 show the differences when $\mathrm{S}$ is set to a lower value.
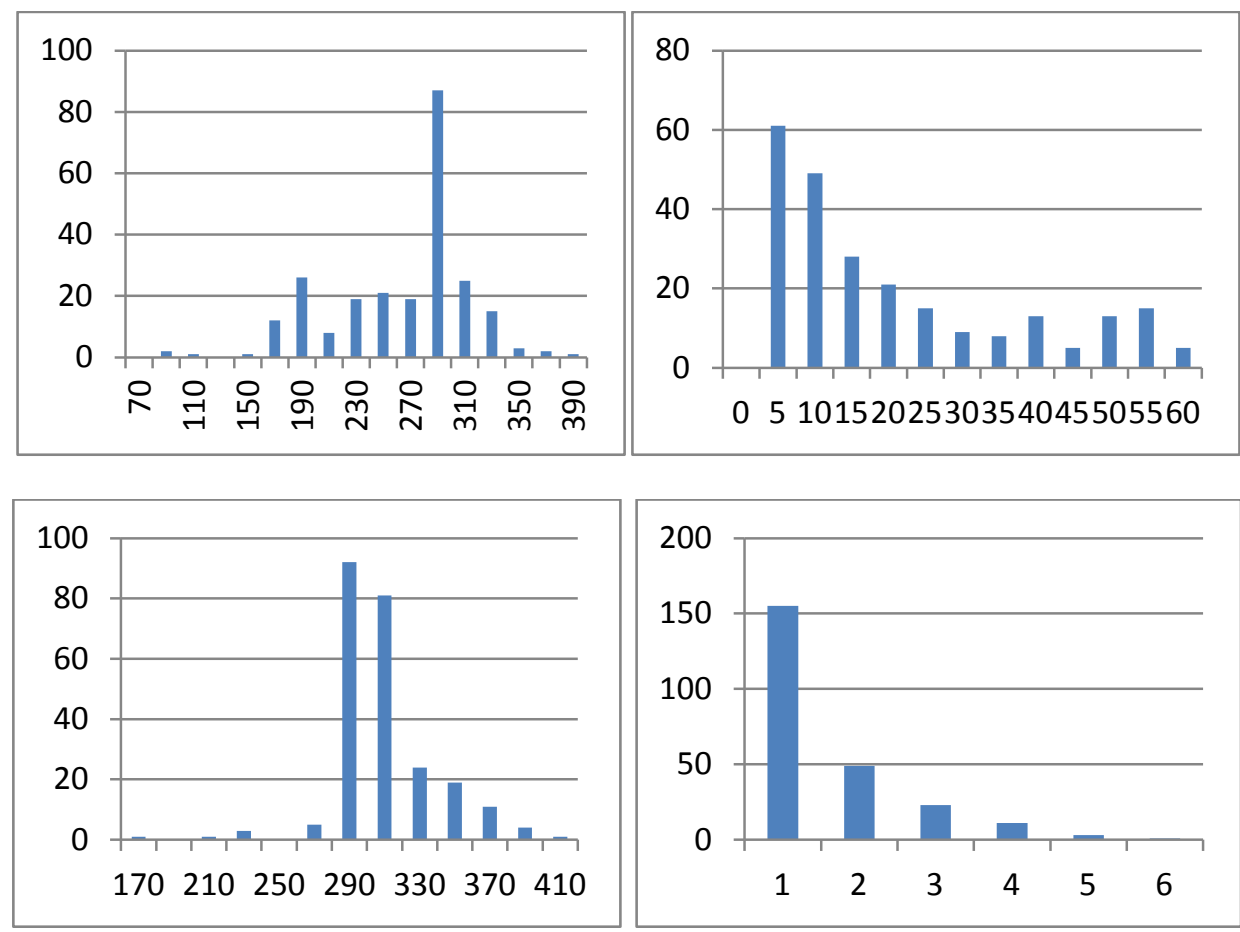

FIGURE 23. (LEFT)TERMINAL (RIGHT)INTERMEDIATE LENGTHS FOR LOWER UNBOUND MAP-2 PRODUCTION

This parameter change again had a surprising impact on the simulation. The intermediate lengths have lowered by around twenty percent and the length of the terminal segments have lengthened by approximately double. The order of branching in the simulation rose by a small amount. This is a much more profound change than raising the value of $\mathrm{S}$ had upon the model. The ratios of the states are still similar to that of the default model, which is why the branching rate is still the same as in the default model. The lengthened terminal segments is hard to explain, it appears that the high value of $c 1$, being greater than the value of $S$, has a massive effect on the amount of unbound MAP-2 converted to bound MAP-2 when the amounts of unbound MAP-2 are very small. 


\begin{tabular}{|l|c|}
\hline Morphological variable & Simulation data \\
\hline Average Terminal Length & $254.01+/-50.82$ \\
\hline Average Intermediate Length & $18.35+/-16.77$ \\
\hline Path Length & $299.48+/-29.92$ \\
\hline Order & $1.6+/-0.96$ \\
\hline
\end{tabular}

Table 18. Statistical data for short terminal tree.

\subsubsection{Overall impact of changing $S$}

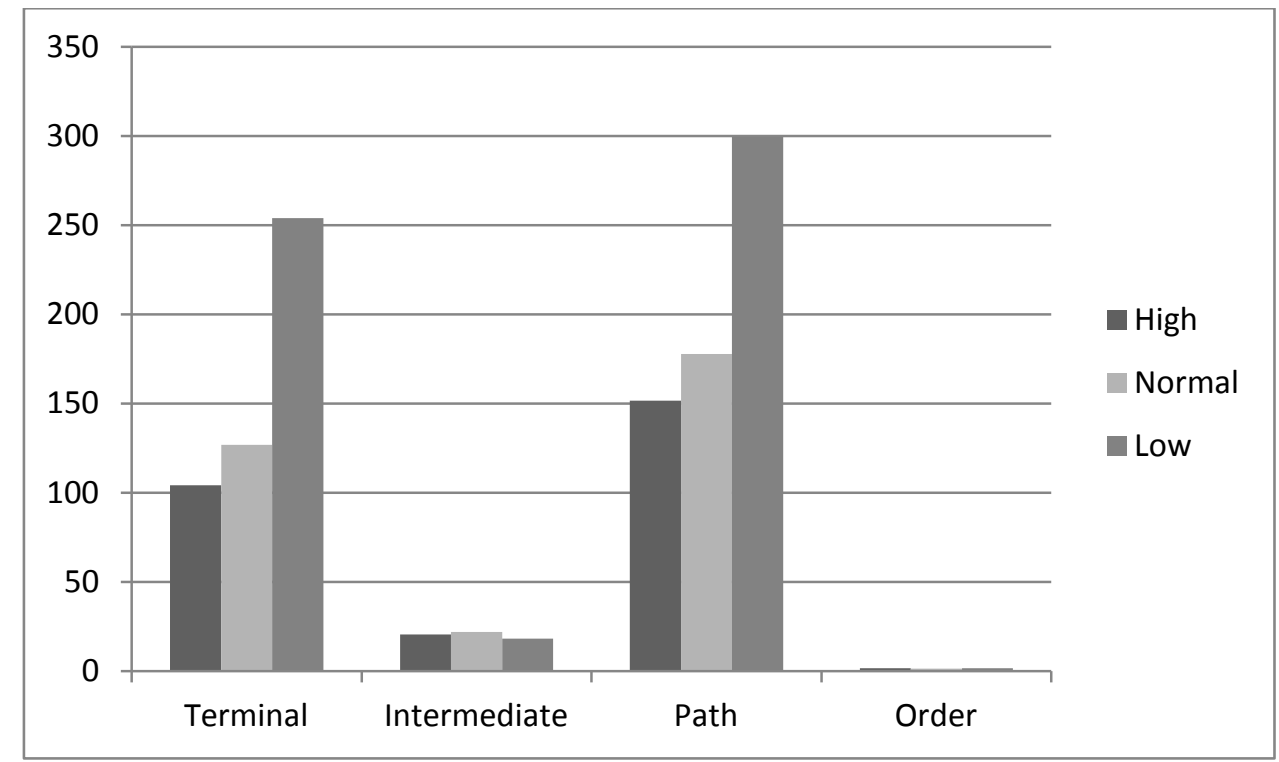

FIGURE 24. OVERALL IMPACT OF CHANGING UNBOUND MAP-2 PRODUCTION

The implication of this data set is that by lowering the amount of unbound MAP-2 in the model, the importance of the elongation phase increases. The general rule of thumb for the model is to ensure that these values are always $\mathrm{c} 1<\mathrm{S}$ to prevent all unbound MAP-2 being instantly converted to bound MAP-2. By lowering the amount of unbound MAP-2 during the branching phase, it allowed more unbound MAP-2 to be available to each growth cone during the elongation phase. Raising the rate of S by fifty percent did not have the expected change in the 
simulation, presumably to the default rate being quite low initially. Raising the rate to a much higher level would then have a greater effect on the model.

\subsubsection{Changing the influx rate for calcium (I)}

The default value for the amount of influx of calcium ensures that the rate of change in the model is correct. By changing this parameter we should be able to alter the timing of the branching point during the simulation and switch much sooner or later from branching to elongation. By increasing the influx rate of calcium which affects every single compartment, the switch between branching and elongation mode of the simulation will likely occur very quickly. I'd expect to see very little branching if any when calcium is increased. When the amount of calcium influx is lowered the reverse should happen, the branching phase of the simulation should last longer and more branching events should occur before elongation takes over.

\subsubsection{Higher influx rate}

Table 19 shows the values when we set the value of I higher than the default.

\begin{tabular}{|l|l|}
\hline Parameter & Value \\
\hline Calcium influx rate & 0.45 \\
\hline
\end{tabular}

Table 19. The higher influx rate for calcium. 
The histograms in figure 25 show the differences when I is set to a higher value.
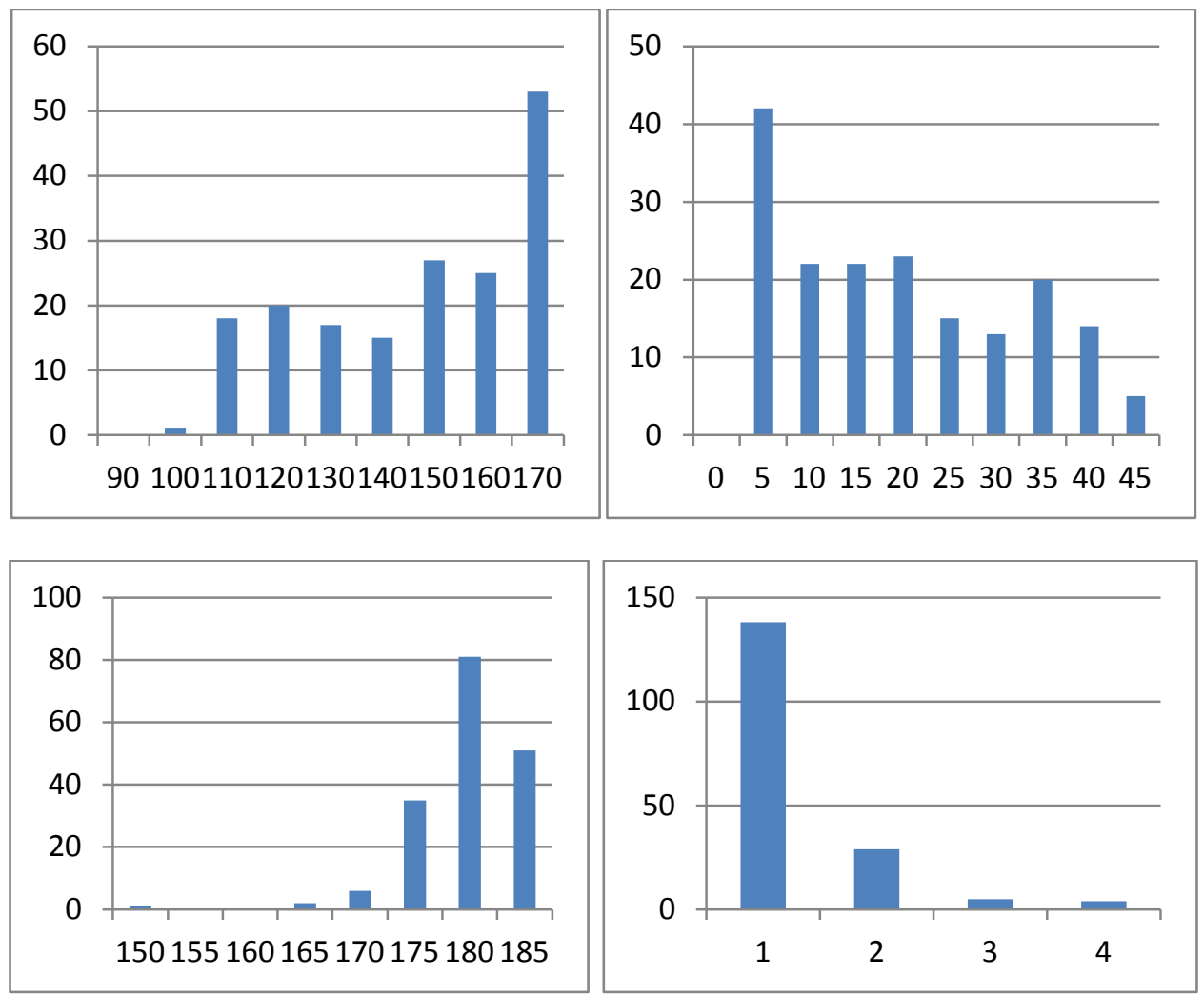

FIGURE 25. (TOP LEFT) TERMINAL (TOP RIGHT) INTERMEDIATE (BOTTOM LEFT) PATH LENGTHS AND (BOTTOM RIGHT) ORDER FOR HIGHER CALCIUM INFLUX

This parameter change alters the phase switching point between branching and elongation in the model happen sooner by flooding the simulation with higher rates of calcium and altering the F and G function appropriately. The order of the branching is lower than the default model which shows that the branching phase is shorter, this is also shown by the intermediate segments being twenty five percent shorter on average than the intermediate segments in the default model. The terminal segments are approximately twenty percent longer which shows that the simulation spends more time in the elongation phase and therefore has more time in order to develop longer terminal segments. 


\begin{tabular}{|l|c|}
\hline Morphological variable & Simulation data \\
\hline Average Terminal Length & $141.79+/-21.73$ \\
\hline Average Intermediate Length & $16.95+/-12.09$ \\
\hline Path Length & $177.1+/-4.35$ \\
\hline Order & $1.28+/-0.63$ \\
\hline
\end{tabular}

Table 20. Statistical data for higher calcium influx tree.

\subsubsection{Lower influx rate}

Table 21 shows the values when we set the value of I lower than the default.

\begin{tabular}{|l|l|}
\hline Parameter & Value \\
\hline Calcium influx rate & 0.15 \\
\hline
\end{tabular}

Table 21. The lower influx rate for calcium.

The histograms in figure 26 show the differences when I is set to a lower value.
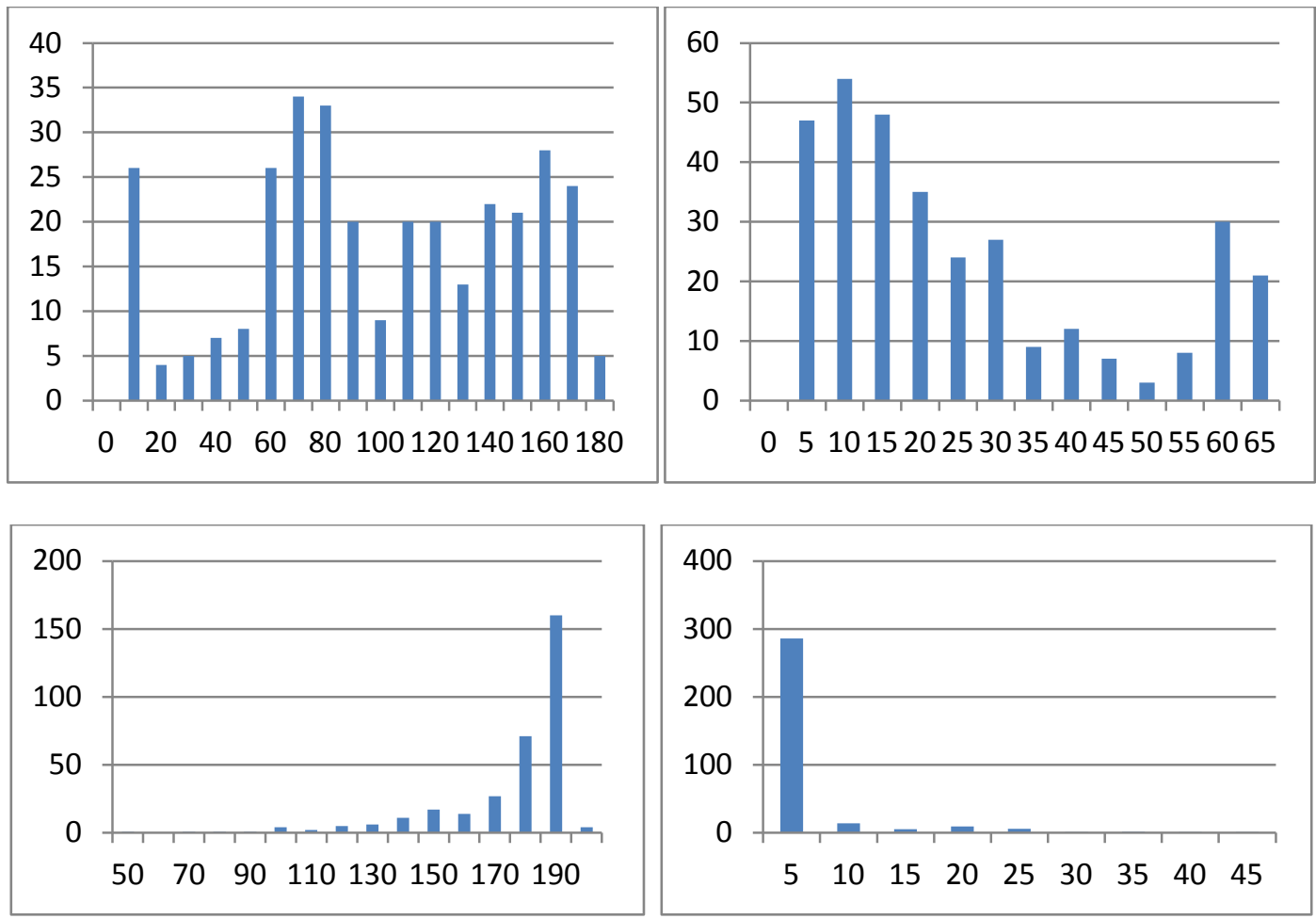

FIGURE 26. (TOP LEFT) TERMINAL (TOP RIGHT) INTERMEDIATE (BOTTOM LEFT) PATH LENGTHS AND (BOTTOM RIGHT) ORDER FOR LOWER CALCIUM INFLUX 
This parameter change alters the phase switching point between branching and elongation in the model happen later by starving the simulation with lower rates of calcium and altering the $\mathrm{F}$ and $G$ function appropriately. The order of the branching has doubled from the value in the default model which shows a much longer branching phase. The intermediate segments have retained the average length of the default model as the calcium changes do not impact on the overall elongation rate which remains highly constant, while there is twice the amount of time for the branches to grow, there is also twice as much time for branching events, and so the overall effect is to keep the average intermediate segment the same length as before. The terminal segments have lost approximately twenty five percent of their length which is consistent with having less time in the elongation phase in order for them to achieve length.

\begin{tabular}{|l|l|}
\hline Morphological variable & Simulation data \\
\hline Average Terminal Length & $94.61+/-48.61$ \\
\hline Average Intermediate Length & $23.24+/-19.44$ \\
\hline Path Length & $171.06+/-22.47$ \\
\hline Order & $3.78+/-5.83$ \\
\hline
\end{tabular}

Table 22. Statistical data for lower calcium influx tree. 


\subsubsection{Overall impact of changing I}

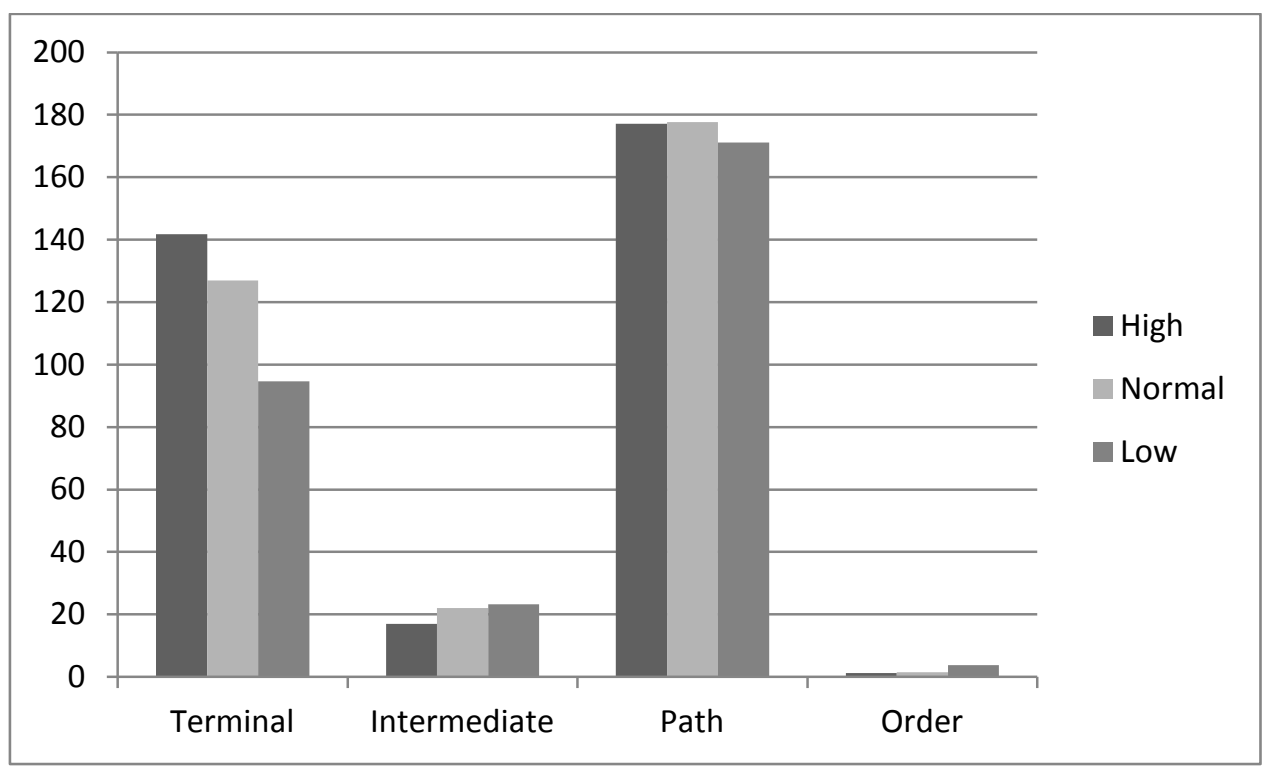

FIGURE 27. OVERALL IMPACT OF CHANGING CALCIUM INFLUX

The effects of changing the amount of calcium influx are to delay or extend the point at which the branching phase of the simulation switches to becoming the elongation phase. By raising and lowering the amount of calcium influx we can alter the point at which $\mathrm{kF}$ and $\mathrm{kG}$ alter the rate change between bound and phosphorylated MAP-2. This leads to different amounts of branching and altered terminal lengths, but the intermediate segments retain their average length when lowering the amount of calcium influx, but significantly shorter when the influx is increased as the branching phase is shortened.

\subsubsection{Changing the transport rate for tubulin $(\eta)$}

The default rate for the transport rate ensures that there is enough tubulin getting to the terminal segments to allow for a decent amount of elongation. By changing this parameter we should be able to alter the overall amount of growth in the simulation. As the default value of the active transport rate is quite small compared to the diffusion rate, its initial impact is negligible, with diffusion flooding compartments with tubulin. Once the amount of tubulin has reached a rough steady state in the simulation with higher concentrations in the proximal end of the dendrite, the 
active transport should move more tubulin than diffusion would towards the distal end during the elongation phase of the simulation. In a model which accurately modelled the number of tubulin molecules rather than the concentration, a more accurate amount of tubulin could be transported by this factor, the reasons why concentrations are not fully modelled is discussed in section 5.4.1. There will be scenarios where there may be less kinesin protein or transport than there is tubulin to transport, providing a lower amount of tubulin to move, which isn't taken into account in this model, the active transport rate is always constant multiplicative feature.

\subsubsection{Higher transport rate}

Table 23 shows the values when we set the value of $\eta$ higher than the default.

\begin{tabular}{|l|l|}
\hline Parameter & Value \\
\hline Active transport rate & 0.045 \\
\hline
\end{tabular}

Table 23. The higher rate for the active transport rate.

The histograms in figure 28 show the differences when $\eta$ is set to a higher value.
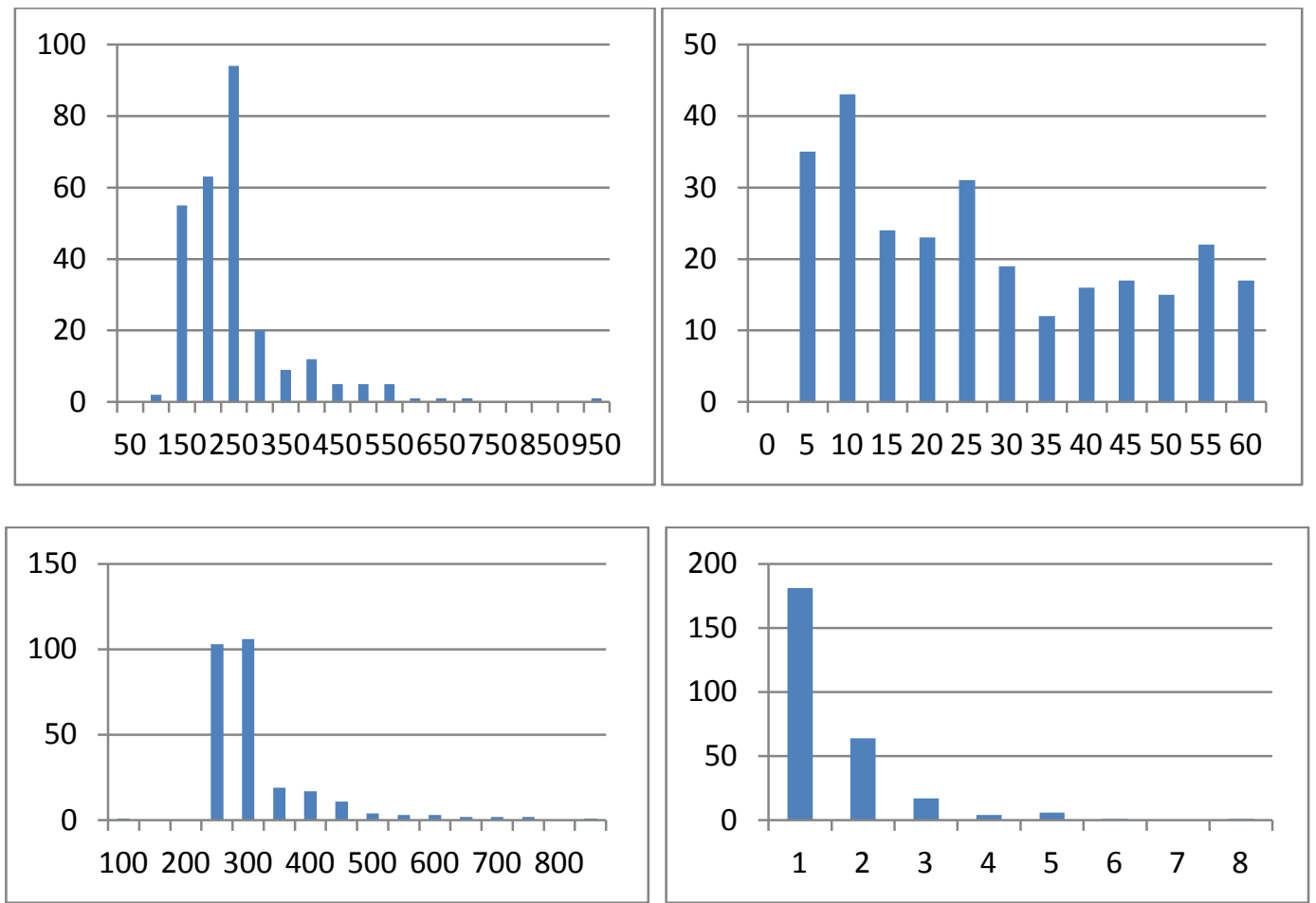

FIGURE 28. (TOP LEFT) TERMINAL (TOP RIGHT) INTERMEDIATE (BOTTOM LEFT) PATH LENGTHS AND (BOTTOM RIGHT) ORDER FOR HIGHER ACTIVE TRANSPORT 
The change in this parameter has had the expected result of increasing the average length of the terminal segments by approximately seventy five percent. These segments have had a long time to grow in the elongation phase, and the higher transport rate has ensured that plenty of tubulin has made it to the growth cones to be assembled into microtubules. The intermediate segments are only marginally longer than the intermediate segments in the default data set. The amount of branching in the data set has not altered at all. The intermediate segments length can be shown by the simulation reaching its tubulin steady state before branching events occur, providing a small benefit to the soon-to-be intermediate segments. After the branching event, the steady state is disrupted and must build up before the active transport again has the fullest effect.

\begin{tabular}{|l|l|}
\hline Morphological variable & Simulation data \\
\hline Average Terminal Length & $230.43+/-106.04$ \\
\hline Average Intermediate Length & $24.82+/-17.64$ \\
\hline Path Length & $291.64+/-95.1$ \\
\hline Order & $1.53+/-0.97$ \\
\hline
\end{tabular}

Table 24. Statistical data for higher active transport tree.

\subsubsection{Lower transport rate}

Table 25 shows the values when we set the value of $\eta$ lower than the default.

\begin{tabular}{|l|l|}
\hline Parameter & Value \\
\hline Active transport rate & 0.015 \\
\hline
\end{tabular}

Table 25. The lower rate for the active transport rate. 
The histograms in figure 29 show the differences when $\eta$ is set to a lower value.
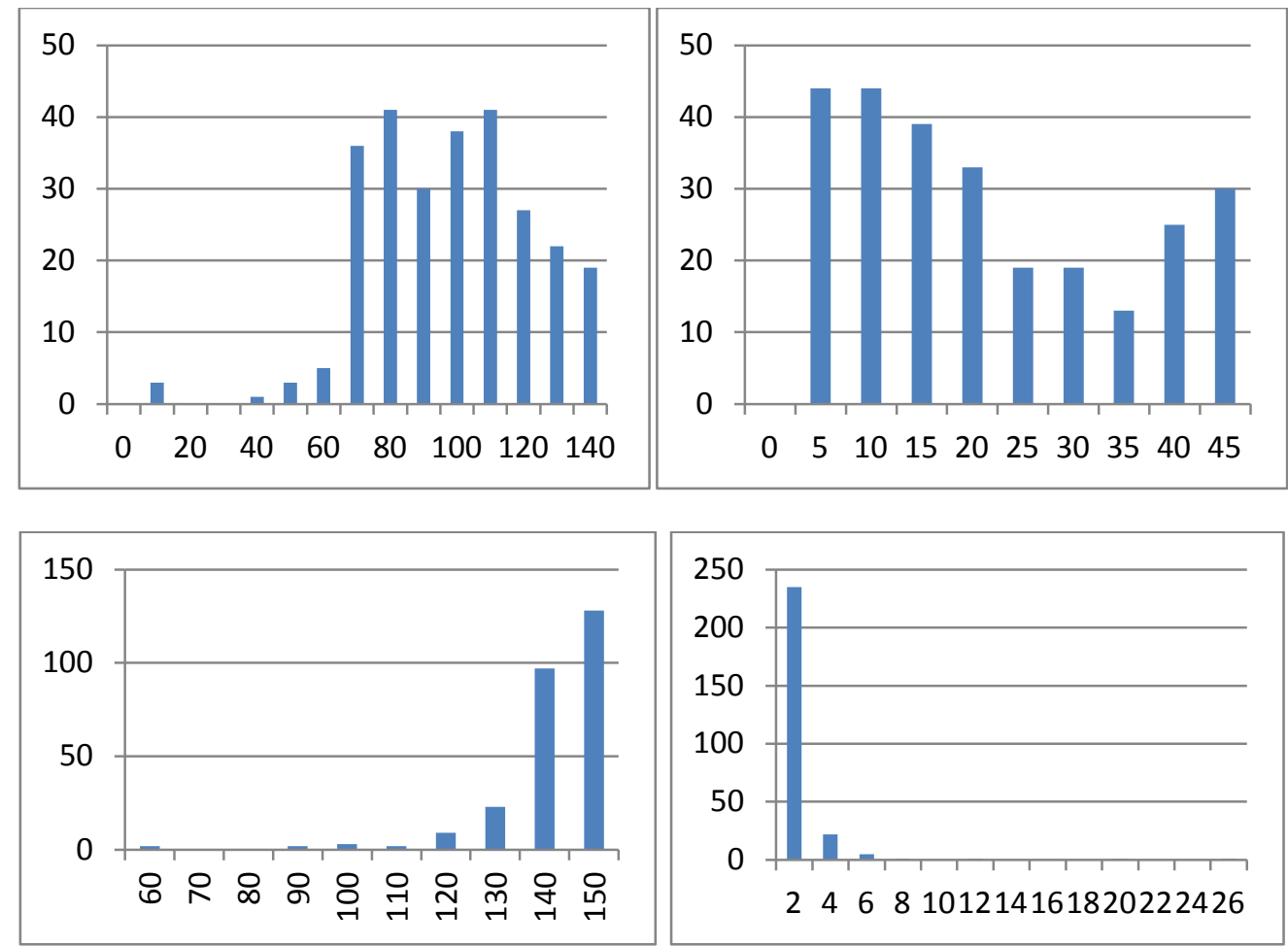

FIGURE 29. (TOP LEFT)TERMINAL (TOP RIGHT)INTERMEDIATE (BOTTOM LEFT) PATH LENGTHS AND (BOTTOM RIGHT) ORDER FOR LOWER ACTIVE TRANSPORT RATE

The change in this parameter has had the expected result of decreasing the average length of the segments in the simulation. The intermediate segments have shortened by fifteen percent showing that while they do rely on the transport of tubulin for some of their length, they are stabilised too soon for the transport to have a large effect upon them. The terminal segments have dropped by just over ten percent of the length of the default model. These spend longer elongating than the intermediate segments, so they rely on an appropriate amount of their length on the active transport rate. The small drop in length is due to diffusion picking up more of the tubulin that would have been transported in the previous time segment, so the free tubulin left by the lowered rate still reaches the growth cone, only delayed in time. 


\begin{tabular}{|l|c|}
\hline Morphological variable & Simulation data \\
\hline Average Terminal Length & $92.85+/-24.41$ \\
\hline Average Intermediate Length & $18.98+/-13.35$ \\
\hline Path Length & $137.71+/-13.36$ \\
\hline Order & $1.76+/-2.1$ \\
\hline
\end{tabular}

Table 26. Statistical data for the lower active transport tree.

\subsubsection{Overall impact of changing $\eta$}

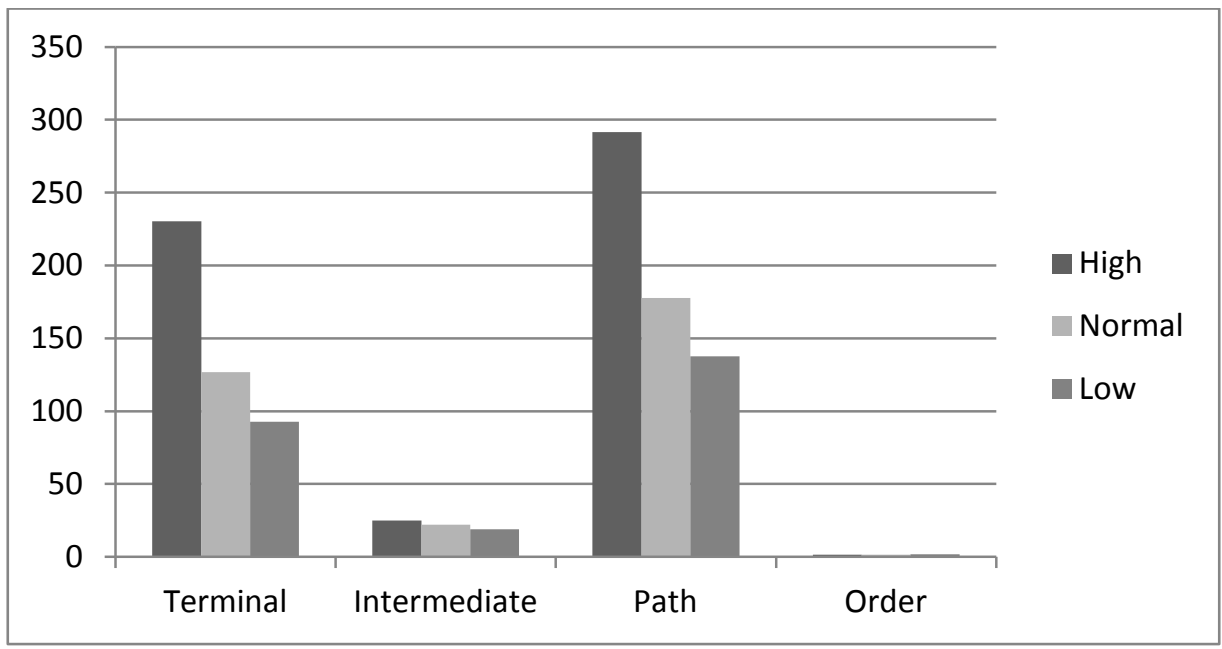

FIGURE 30. OVERALL IMPACT OF CHANGING ACTIVE TRANSPORT RATE

The impact of the changing the active transport rate has a greater effect the larger the difference. As active transport is working in conjunction with diffusion to bring tubulin from the proximal to distal ends of the dendrite, it provides an additive effect on the amount of tubulin transported. If the amount of transport is small it has a small effect on the overall length of the dendrite, however if the amount of transport is large, the effect on the overall length is huge. The amount of transport in the default model is an excellent middle ground for this rate, however the amount of transport in the default model has little overall effect, diffusion does the majority of the work of transporting tubulin. 


\subsubsection{Changing the diffusion rate (D)}

The default diffusion rate makes sure all the proteins flow through the tree. Changing this parameter will have the most profound effect on the amount of unbound MAP-2 in the model. Calcium has influx throughout the tree, tubulin is actively transported by kinesin proteins, however MAP-2 relies on diffusion to reach the terminal, where its state is altered from the soma where it is produced. Changing this value should have an effect on the amount of elongation in the model, as by starving the terminal segments of unbound MAP-2, and therefore bound MAP-2, there will be little tubulin binding happening. As branching rates remain a proportion of the states of MAP-2, this should be directly unaffected. It will be interesting to see whether the starving of unbound MAP-2 has a similar effect on the simulation as reducing the production rate of unbound MAP-2

\subsubsection{Higher diffusion rate}

Table 27 shows the values when we set the value of $\mathrm{D}$ higher than the default.

\begin{tabular}{|l|l|}
\hline Parameter & Value \\
\hline Diffusion rate & 1.5 \\
\hline
\end{tabular}

Table 27. The higher diffusion rate. 
The histograms in figure 31 show the differences when $\mathrm{D}$ is set to a higher value.
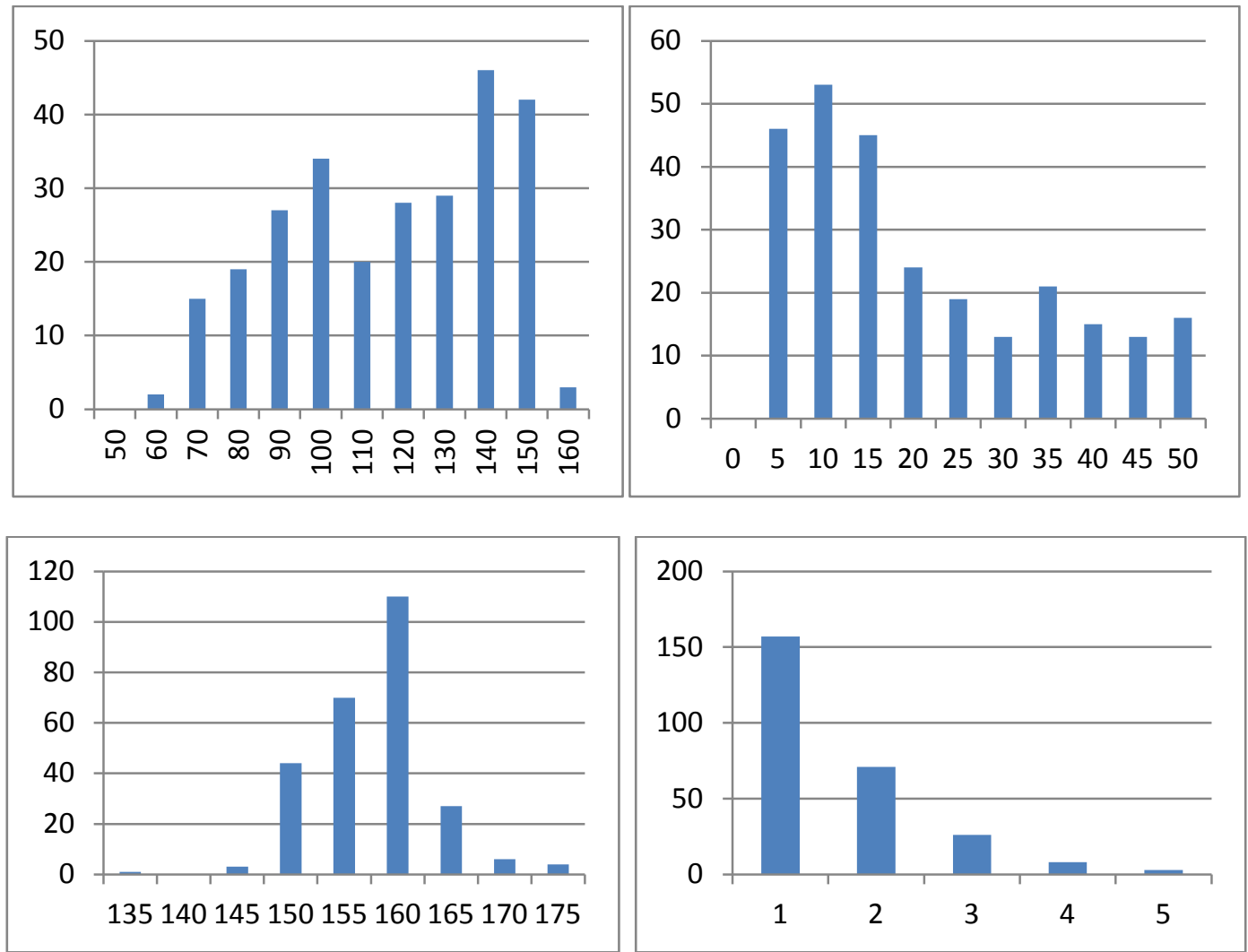

FIGURE 31. (TOP LEFT)TERMINAL (TOP RIGHT)INTERMEDIATE (BOTTOM LEFT) PATH LENGTHS AND (BOTTOM RIGHT) ORDER FOR HIGHER DIFFUSION RATE

The change in this parameter allows concentrations of molecules flow from one compartment to another at a higher rate. By increasing the rate of diffusion the length of the segments is longer as expected. The terminal segments increased in length by twenty percent as a larger amount of tubulin and MAP-2 reaches the distal ends of the dendrite. The increase in the rate of calcium flowing to the tips will have hastened the end of the branching phase slightly resulting in a very slight drop in length for the intermediate segments. The slight drop in length is explained by an increase in the elongation of the intermediate segments while they are still terminal segments, but the shorter branching phase gives them less time to use that increased rate of growth. 


\begin{tabular}{|l|c|}
\hline Morphological variable & Simulation data \\
\hline Average Terminal Length & $112.46+/-25.71$ \\
\hline Average Intermediate Length & $18.04+/-13.74$ \\
\hline Path Length & $155.25+/-5.18$ \\
\hline Order & $1.6+/-0.86$ \\
\hline
\end{tabular}

Table 28. Statistical data for higher diffusion tree.

\subsubsection{Lower diffusion rate}

Table 29 shows the values when we set the value of $\mathrm{D}$ lower than the default.

\begin{tabular}{|l|l|}
\hline Parameter & Value \\
\hline Diffusion rate & 0.5 \\
\hline
\end{tabular}

Table 29. The lower diffusion rate.

The histograms in figure 32 show the differences when $\mathrm{D}$ is set to a lower value.
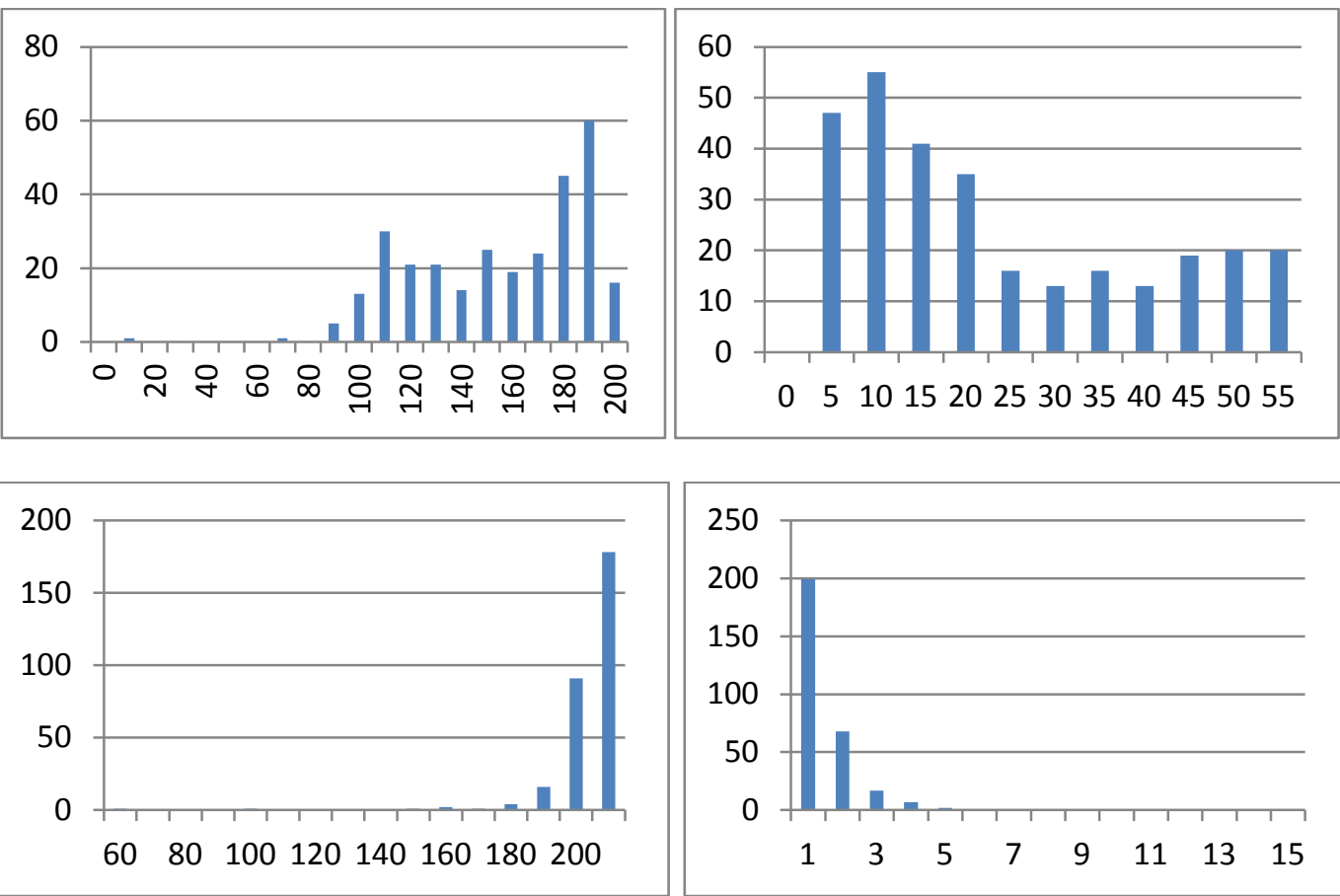

FIGURE 32. (TOP LEFT) TERMINAL (TOP RIGHT) INTERMEDIATE (BOTTOM LEFT) PATH LENGTHS AND (BOTTOM RIGHT) ORDER FOR LOWER DIFFUSION RATE 
The change from this parameter is as expected to reduce the amount of overall length in the dendrite. The intermediate segment average length dropped by approximately twenty percent compared to the default model. The terminal segments average length dropped by approximately ten percent. This is quite a minor reduction showing that the model has enough ways of getting chemicals to the distal tips in order to keep the model moving. The relatively small amounts of unbound MAP-2 had less of an effect due to the lack of retraction in the model, with retraction added into the model, the length of the segments would be shorter and would likely reach a relatively short steady state where the amount of tubulin and MAP-2 entering the terminal compartments was only sufficient to sustain the microtubules already constructed. The order was slightly increased in the lowered version, caused by the amount of bound MAP-2 that was being used to sustain the already bound tubulin leaving a greater ratio of phosphorylated MAP-2 for branching. The unbound MAP-2 effect did not come into play, suggesting that the link between the production rate and the c1 constant is stronger than purely the lack of unbound MAP-2 in the simulation.

\begin{tabular}{|l|l|}
\hline Morphological variable & Simulation data \\
\hline Average Terminal Length & $150.66+/-33.56$ \\
\hline Average Intermediate Length & $20.82+/-16.2$ \\
\hline Path Length & $198.02+/-12.72$ \\
\hline Order & $1.51+/-1.16$ \\
\hline
\end{tabular}

Table 30. Statistical data for lower diffusion tree. 


\subsubsection{Overall impact of changing $D$}

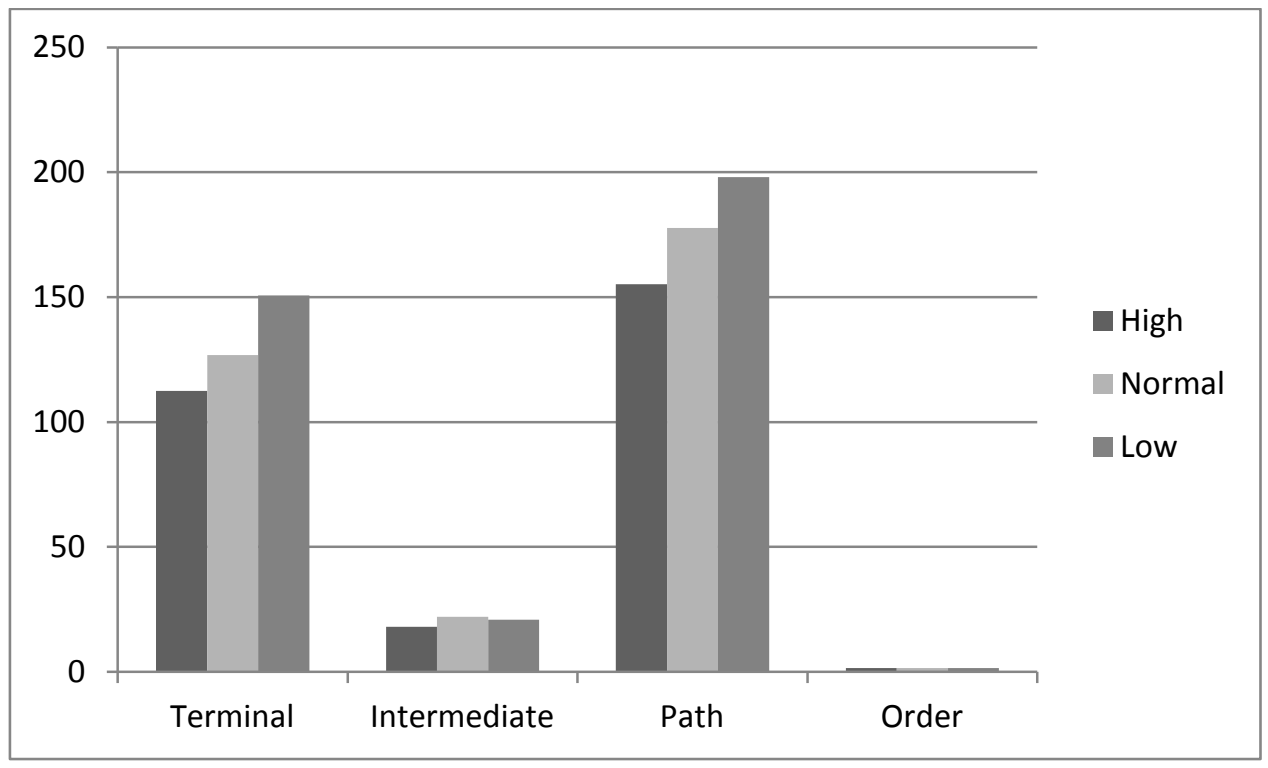

FIGURE 33. OVERALL IMPACT OF CHANGING THE DIFFUSION CONSTANT

The overall impact is muted when diffusion is lowered due to the lack of retraction in the model, it shows that diffusion (obviously) plays an important role in the overall length of the model, but only indirectly affects the branching potentials through the MAP-2 levels diffused into the growth cone. The impact of increasing the rate of diffusion is to marginally speed up the time the simulation takes to swap from a branching state to an elongation state initially, and has an excitatory effect on the overall length of the terminal segments.

\subsubsection{Changing the conversion constants (c1, c2, c3 c4)}

Changing the conversion constants has a massive effect on the morphology of the neurite. They flatten or steepen the rate of change between the states of MAP-2 once the switching point is passed. A higher c4 than c3, which is the default, will mitigate the effects of a branching phase somewhat, ensuring there is still a large enough amount of bound MAP-2 to allow elongation, and once the neurite is switched into its elongation phase, it ensures that the majority of dephosphorylated MAP-2 is converted into bound MAP-2 for a healthy elongation phase. 
Changing these constants even slightly then, can have a massive effect on the outcome of the morphology of the simulation.

\subsubsection{Higher c1}

It is expected that by raising $\mathrm{c} 1$, more unbound MAP-2 will become bound and so raise the elongation rate. Table 31 shows the values when we set the value of $\mathrm{c} 1$ higher than the default.

\begin{tabular}{|l|l|}
\hline Parameter & Value \\
\hline c1 & 1.0 \\
\hline
\end{tabular}

Table 31. The higher c1.

The histograms in figure 34 show the differences when c1 is set to a higher value.
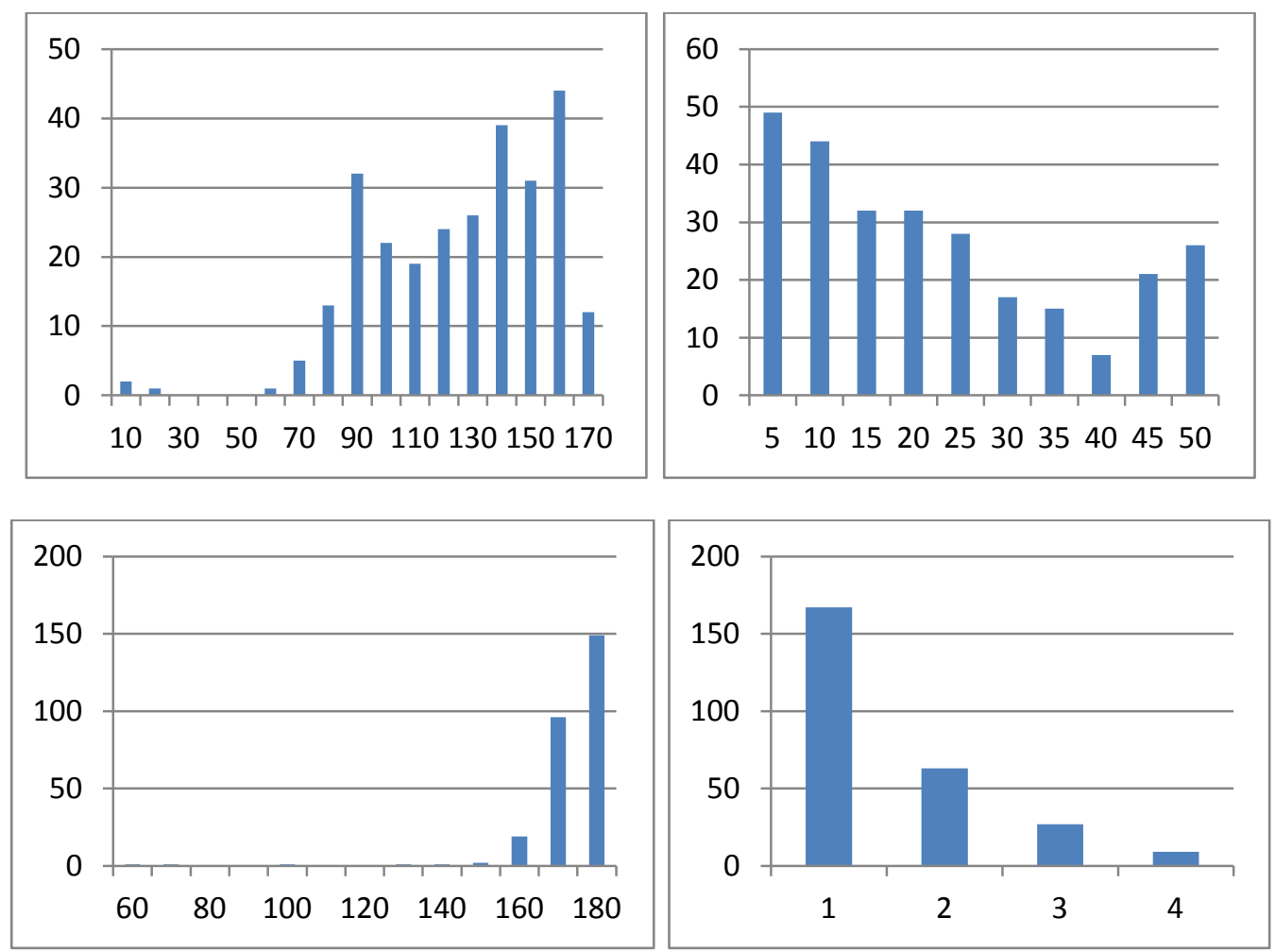

FIGURE 34. (TOP LEFT) TERMINAL (TOP RIGHT) INTERMEDIATE (BOTTOM LEFT) PATH LENGTHS AND (BOTTOM RIGHT) ORDER FOR HIGHER C1

By changing this parameter so high, there was the expected slight unexpected result that the intermediate segments lost ten percent of their length against the default data set. The terminal 
segments also lost approximately ten percent of their length against the reference data set. The amount of unbound MAP-2 being altered into bound MAP-2 is likely to keep the actual levels of MAP-2 very low which would mitigate against the effect of trying to effectively create MAP-2 from nowhere. The actual levels of bound MAP-2 and phosphorylated MAP-2 would be relatively constant once the model had reached a steady state so the drop in length is likely a correctional event by the marginally increased bound MAP-2 converting back into unbound MAP-2 at a slightly higher rate due to the over conversion.

\begin{tabular}{|l|c|}
\hline Morphological variable & Simulation data \\
\hline Average Terminal Length & $121.05+/-30.45$ \\
\hline Average Intermediate Length & $19.66+/-14.75$ \\
\hline Path Length & $167.81+/-12.56$ \\
\hline Order & $1.77+/-2.41$ \\
\hline
\end{tabular}

Table 32. Statistical data for lower c1 tree.

\subsubsection{Lower c1}

By lowering $\mathrm{c} 1$ we are expecting the overall length of the segments to drop, but not by too much and for the branching rate to remain unaffected. By lowering c1 there is less unbound MAP-2 being converted into bound MAP-2, while still retaining high levels of unbound MAP2. Table 33 shows the values when we set the value of $\mathrm{c} 1$ lower than the default.

\begin{tabular}{|l|l|}
\hline Parameter & Value \\
\hline c1 & 0.4 \\
\hline
\end{tabular}

Table 33. The lower c1. 
The histograms in figure 35 show the differences when c1 is set to a lower value.
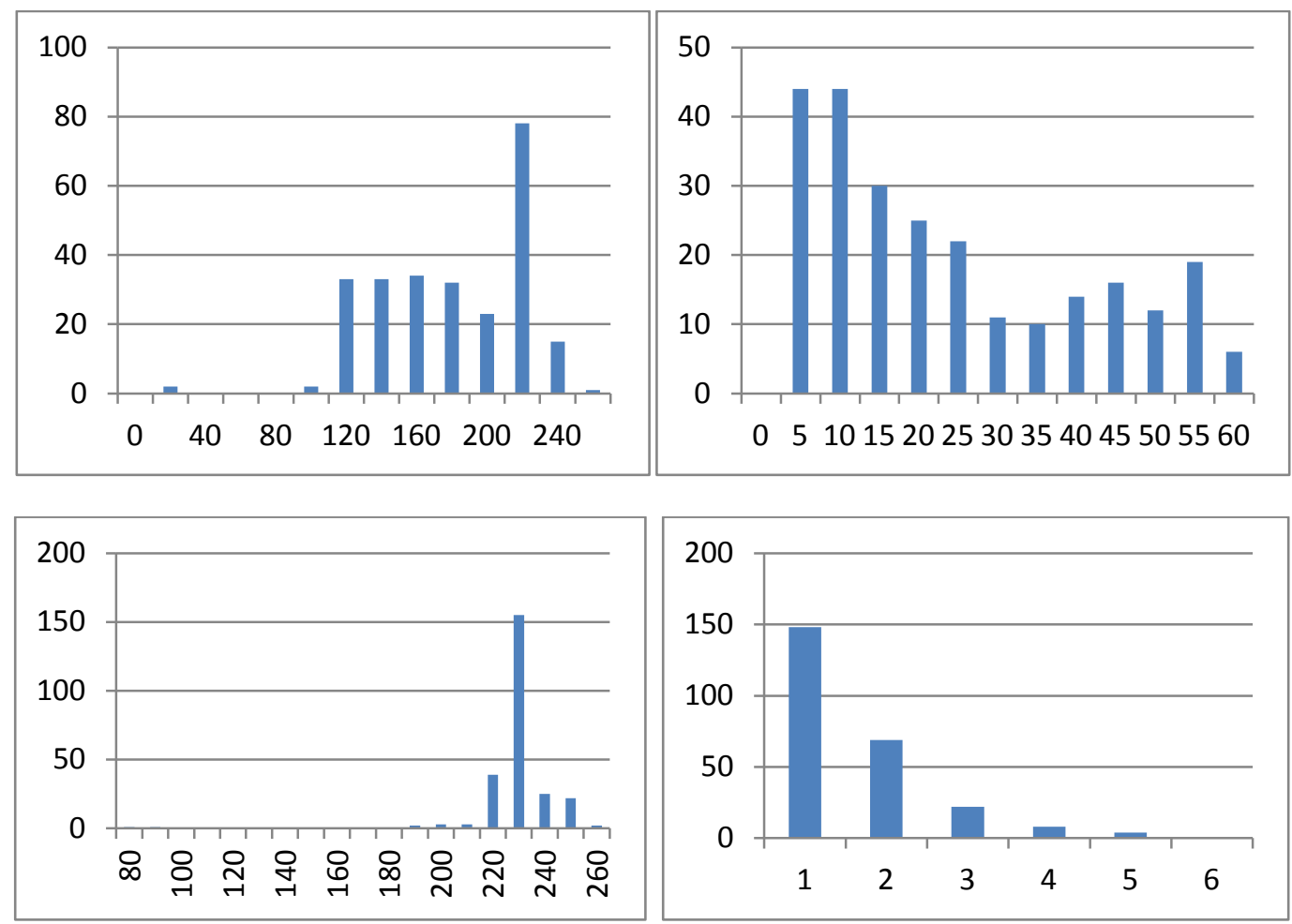

FIGURE 35. (TOP LEFT) TERMINAL (TOP RIGHT) INTERMEDIATE (BOTTOM LEFT) PATH LENGTHS AND (BOTTOM RIGHT) ORDER FOR LOWER C1

By changing this parameter we are altering the rate at which unbound MAP-2 is converted into bound MAP-2 and so mitigating the effects of the phase change due to F and G finally equalising, this results in the branching order being slightly higher as the higher phosphorylated MAP-2 ratio lasts longer, but the intermediate segments retain the same length as the default data set as the elongation in the branching phase remains constant for the entire time the branching phase is extended. The average terminal length has increased by fifty percent however as the effects of unbound MAP-2 are mitigated by a higher swing of MAP-2 from phosphorylated to bound, producing a greater elongation effect once the elongation phase starts. The build up of unbound MAP-2 once a steady state is reached from the initial burst of activity from the switch from phosphorylated to bound increases until it produces a lesser rate of regular growth but enough to ensure the length of the terminal segments is seriously increased. 


\begin{tabular}{|l|l|}
\hline Morphological variable & Simulation data \\
\hline Average Terminal Length & $171.27+/-41.89$ \\
\hline Average Intermediate Length & $21.4+/-16.93$ \\
\hline Path Length & $224.68+/-16.34$ \\
\hline Order & $1.77+/-2.06$ \\
\hline
\end{tabular}

Table 34. Statistical data for lower c1 tree.

\subsubsection{Higher c2}

By increasing c2 we are removing more bound MAP-2, and turning it into unbound MAP-2. This should have the effect of shortening the entire tree and raising the branching order somewhat as the rate of change between the branching to elongation states will have been inhibited by slowing down the conversion of unbound MAP-2 into bound MAP-2 which was mitigating the high $\mathrm{F}$ function during the branching phase. Table 35 shows the values when we set the value of c2 higher than the default.

\begin{tabular}{|l|l|}
\hline Parameter & Value \\
\hline c2 & 0.15 \\
\hline
\end{tabular}

Table 35. The higher c2. 
The histograms in figure 36 show the differences when c2 is set to a higher value.
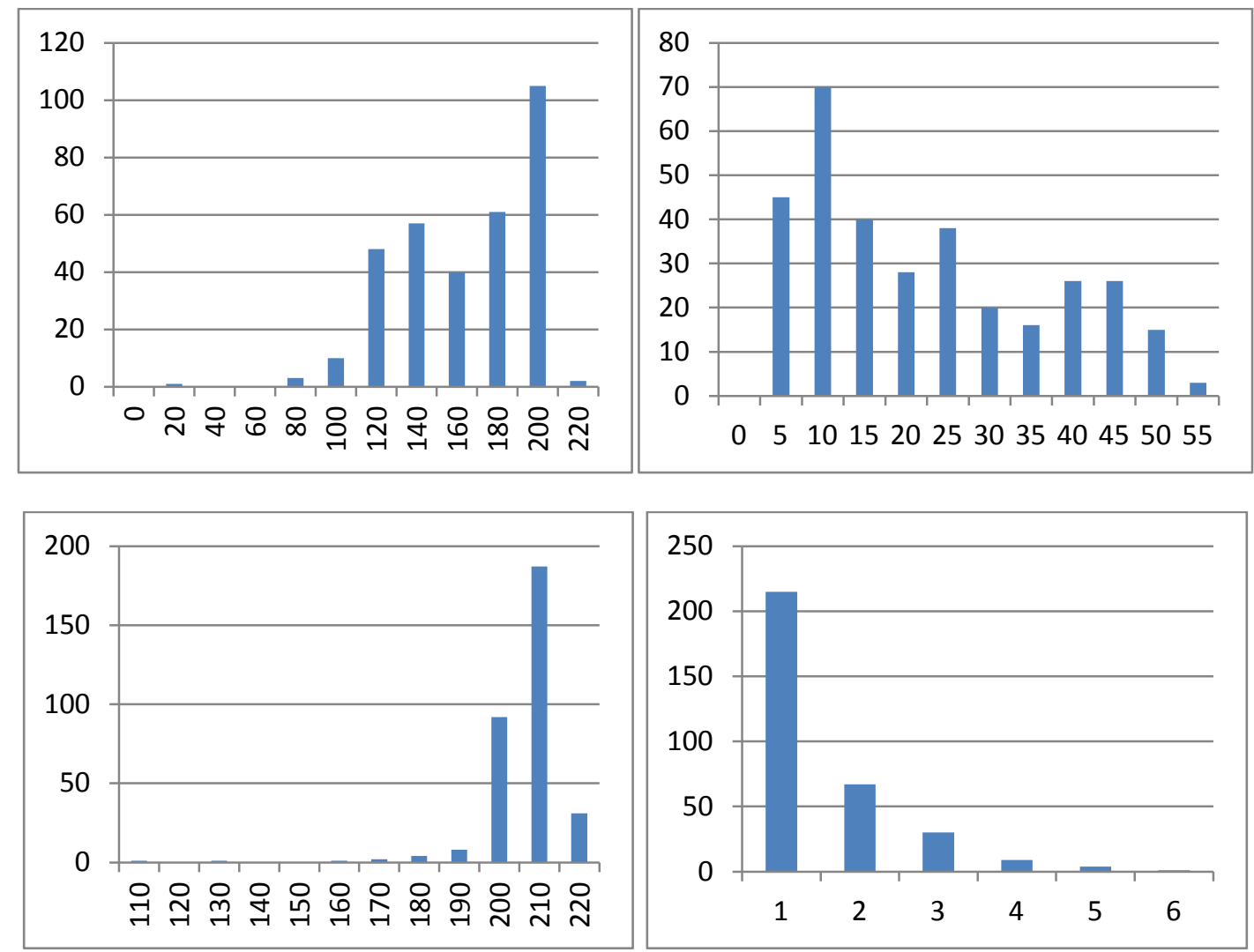

FIGURE 36. (TOP LEFT) TERMINAL (TOP RIGHT) INTERMEDIATE (BOTTOM RIGHT) PATH LENGTHS AND (BOTTOM RIGHT) ORDER FOR HIGHER C2

By changing this parameter we are limiting the amount of unbound MAP-2 that is being altered into bound MAP-2. As expected, the order of the branches increased slightly, and the intermediate segments average length was ten percent shorter than the intermediate segments in the default data set. The terminal segments have increased in length by approximately twenty percent as the effects of unbound MAP-2 are mitigated by a higher swing of MAP-2 from phosphorylated to bound, producing a greater elongation effect once the elongation phase starts. The build up of unbound MAP-2 once a steady state is reached from the initial burst of activity from the switch from phosphorylated to bound increases until it produces a lesser rate of regular growth but enough to ensure the length of the terminal segments is increased. This is similar to the effect of lower the c1 constant. 


\begin{tabular}{|l|l|}
\hline Morphological variable & Simulation data \\
\hline Average Terminal Length & $155.53+/$ - 33.61 \\
\hline Average Intermediate Length & $19.55+/-14.1$ \\
\hline Path Length & $201.24+/-10.27$ \\
\hline Order & $1.62+/-1.76$ \\
\hline
\end{tabular}

Table 36. Statistical data for higher c2 tree.

\subsubsection{Lower c2}

By lowering c2 we are decreasing the amount of bound MAP-2 that is converted into unbound MAP-2. Given the similarity between the lowering of $\mathrm{c} 1$ and the raising of c2, it is likely that the lowering of c2 will have a similar effect to that of raising c1. Table 37 shows the values when we set the value of c2 lower than the default.

\begin{tabular}{|l|l|}
\hline Parameter & Value \\
\hline c2 & 0.05 \\
\hline
\end{tabular}

Table 37. The lower c2. 
The histograms in figure 37 show the differences when c2 is set to a lower value.
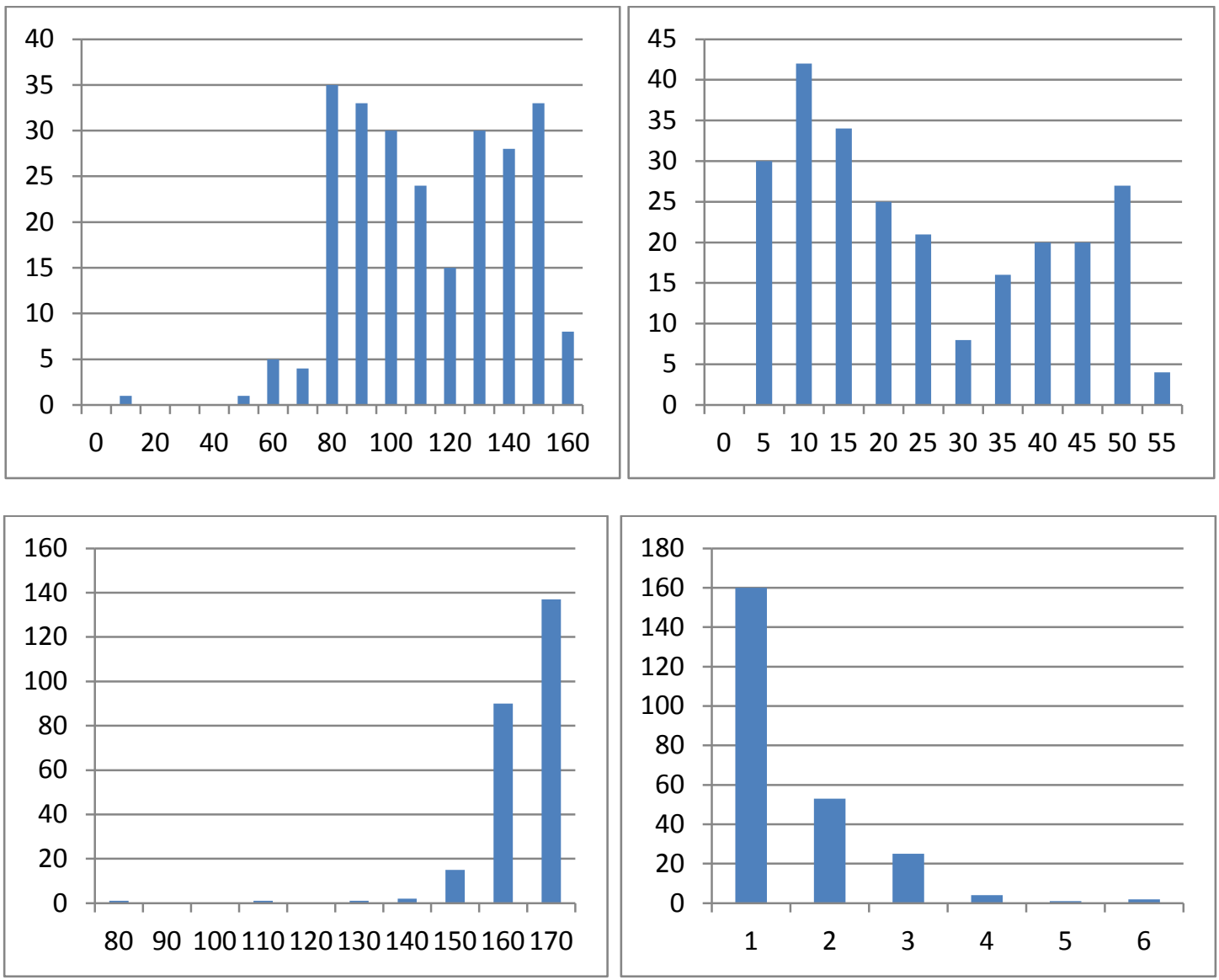

FIGURE 37. (TOP LEFT) TERMINAL (TOP RIGHT) INTERMEDIATE (BOTTOM LEFT) PATH LENGTHS AND (ORDER) FOR LOWER C2

By lowering this parameter we are decreasing the amount of bound MAP-2 which is being released into unbound MAP-2. As expected the order of the branching raised slightly from the branching order in the default set of data. The intermediate lengths remained the same as the intermediate lengths in the default data set. The average terminal lengths shortened by ten percent from the terminal segments in the default data set. The reason the values for lowering c2 are different from the ones gathered when raising c1 are due to the lower concentrations of bound MAP-2 and phosphorylated MAP-2 compared to that of the unbound MAP-2. As the values are much lower, the effect of the less bound MAP-2 being converted is lowered. This is due to the constant working against a ratio of the bound MAP-2. As the values for unbound MAP-2 are much higher than those for bound MAP-2 changing c1 has more of an effect than changing $\mathrm{c} 2$. 


\begin{tabular}{|l|l|}
\hline Morphological variable & Simulation data \\
\hline Average Terminal Length & $108.1+/-27.45$ \\
\hline Average Intermediate Length & $22.22+/-15.55$ \\
\hline Path Length & $160.55+/-9.89$ \\
\hline Order & $1.59+/-1.18$ \\
\hline
\end{tabular}

Table 38. Statistical data for short terminal tree.

\subsubsection{Higher c3}

By increasing c3 we are increasing the amount of bound MAP-2 that gets altered into phosphorylated MAP-2. By raising this, we should delay the rate of change when the F and G functions are aligned, and lengthen the amount of time the branching phase has. It is likely the overall growth of the tree will be greatly shorter, especially the terminal segments and that there is a higher branching order. Table 39 shows the values when we set the value of c3 higher than the default.

\begin{tabular}{|l|l|}
\hline Parameter & Value \\
\hline c3 & 0.15 \\
\hline
\end{tabular}

Table 39. The higher c3. 
The histograms in figure 38 show the differences when c3 is set to a higher value.
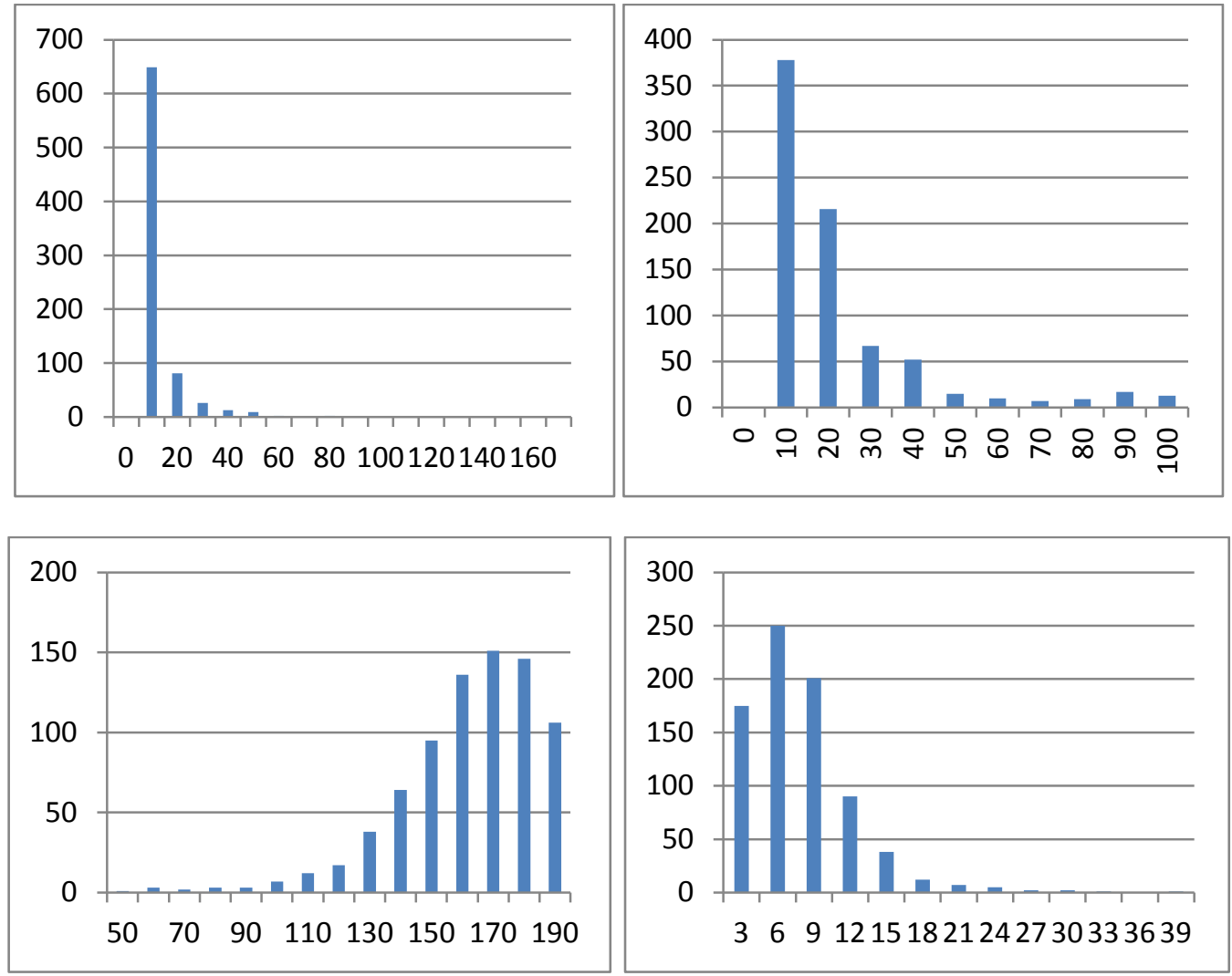

FIGURE 38. (TOP LEFT) TERMINAL (TOP RIGHT) INTERMEDIATE (BOTTOM LEFT) PATH LENGTHS AND (BOTTOM RIGHT) ORDER FOR HIGHER C3

By increasing this parameter, we are increasing the amount of bound MAP-2 that is altered to become phosphorylated MAP-2. As expected there is a fourfold increase in the branching order compared to the default model's data set. The intermediate segments are twenty percent shortened than the intermediate segments in the default data model. The terminal segments are only five percent of the terminal segments in the default data set. This is due to the branching phase of the simulation lasting the whole length of the life time of the simulation. The F and G function's alignment is flattened by the high amount of bound MAP-2 that becomes phosphorylated MAP-2, meaning that the bound MAP-2 never dominates enough to produce a very low branching probability. As the elongation of the terminal segments stalls due to a lack of bound MAP-2 and reaches a steady state in length, it continues to branch with little elongation of these branches and so producing the very short terminal segments. 


\begin{tabular}{|l|l|}
\hline Morphological variable & Simulation data \\
\hline Average Terminal Length & $6.9+/-10.92$ \\
\hline Average Intermediate Length & $17.54+/-19.26$ \\
\hline Path Length & $156.73+/-22.9$ \\
\hline Order & $6.83+/-4.44$ \\
\hline
\end{tabular}

Table 40. Statistical data for higher c3 tree.

\subsubsection{Lower c3}

By decreasing c3 we are decreasing the amount of bound MAP-2 that gets altered into phosphorylated MAP-2. By lowering this, we should increase the rate of change when the $\mathrm{F}$ and $\mathrm{G}$ functions are aligned, and shorten the amount of time the branching phase has. It is likely the overall growth of the tree will be lengthened and that there is a lower branching order. Table 41 shows the values when we set the value of c3 lower than the default.

\begin{tabular}{|l|l|}
\hline Parameter & Value \\
\hline c3 & 0.05 \\
\hline
\end{tabular}

Table 41. The lower c3. 
The histograms in figure 39 show the differences when c3 is set to a lower value.
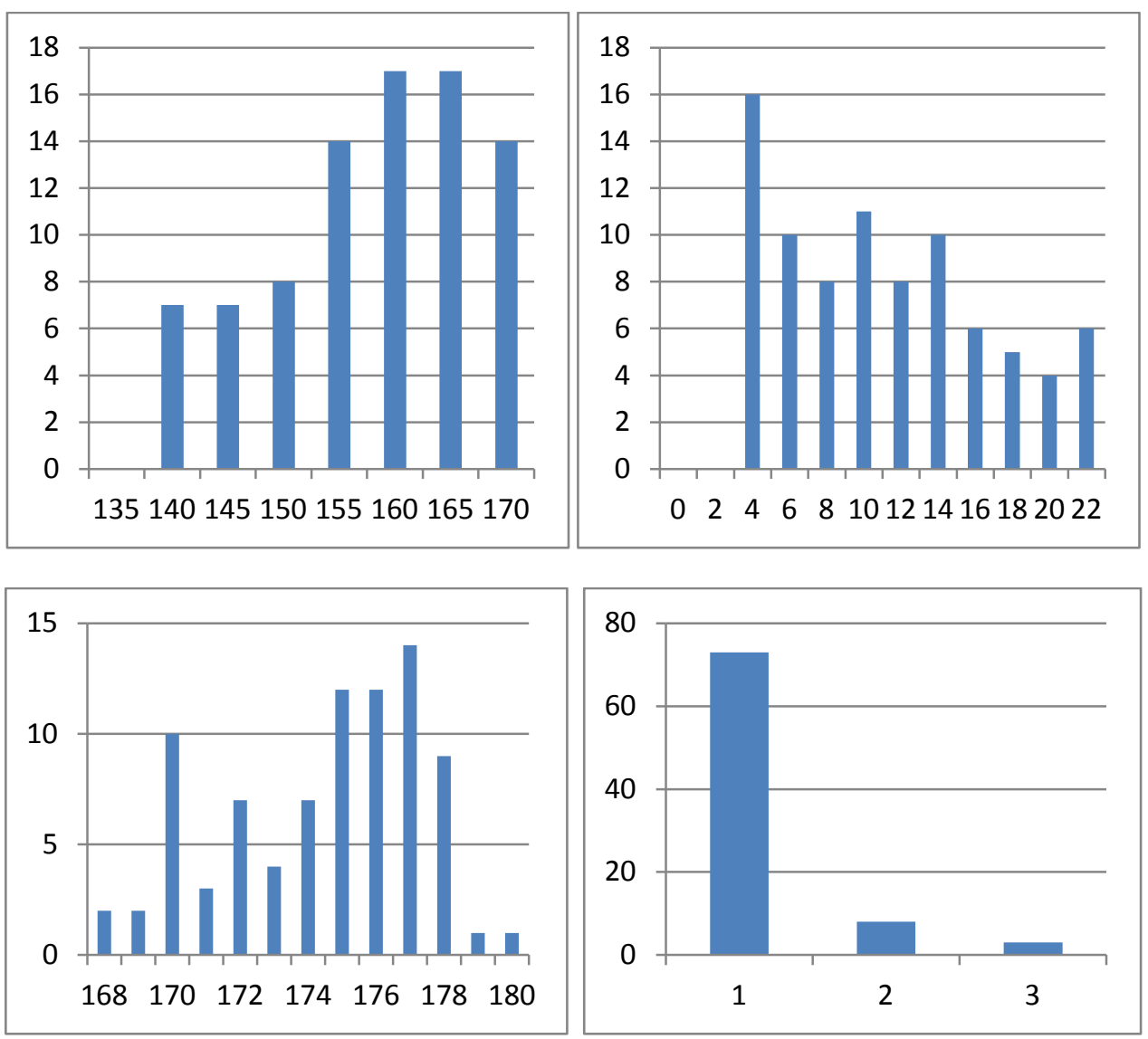

FIGURE 39. (TOP LEFT) TERMINAL (TOP RIGHT) INTERMEDIATE (BOTTOM LEFT) PATH LENGTHS AND (BOTTOM RIGHT) ORDER FOR LOWER C3

By decreasing this parameter, we are decreasing the amount of bound MAP-2 that is altered to become phosphorylated MAP-2. As expected the branching order has lowered by thirty percent. The intermediate segments are much shorter losing almost sixty percent of their length compared to the intermediate segments in the default data set. The terminal segments are thirty percent longer than the terminal segments in the default data set. The overall path length is approximately the same as the average path lengths in the default data set. The short intermediate segment length is due to the shortened transition time from branching state to elongation state of the simulation. The longer terminal segments are more due to the increased time they have to grow than the shift of phosphorylated MAP-2 into bound MAP-2. The amounts, once the elongation stage has been reached are quite low, and so changing this in favour of the bound state has less of an effect. 


\begin{tabular}{|l|l|}
\hline Morphological variable & Simulation data \\
\hline Average Terminal Length & $155.4+/-8.82$ \\
\hline Average Intermediate Length & $9.58+/-5.63$ \\
\hline Path Length & $173.91+/-2.92$ \\
\hline Order & $1.16+/-0.46$ \\
\hline
\end{tabular}

Table 42. Statistical data for lower c3 tree.

\subsubsection{Higher c4}

By changing the $\mathrm{c} 4$ parameter, we are altering the rate at which the phosphorylated MAP-2 is altered to become bound MAP-2. By increasing the c4 parameter we are increasing the amount that is altered, and should increase the rate of change when the $F$ and $G$ functions have equalised, and shorten the amount of time the branching phrase has. It is likely that the overall growth of the tree will be lengthened and that there is a lower branching order. Table 43 shows the values when we set the value of c4 higher than the default.

\begin{tabular}{|l|l|}
\hline Parameter & Value \\
\hline c4 & 1.5 \\
\hline
\end{tabular}

Table 43. The higher c4. 
The histograms in figure 40 show the differences when c4 is set to a higher value.
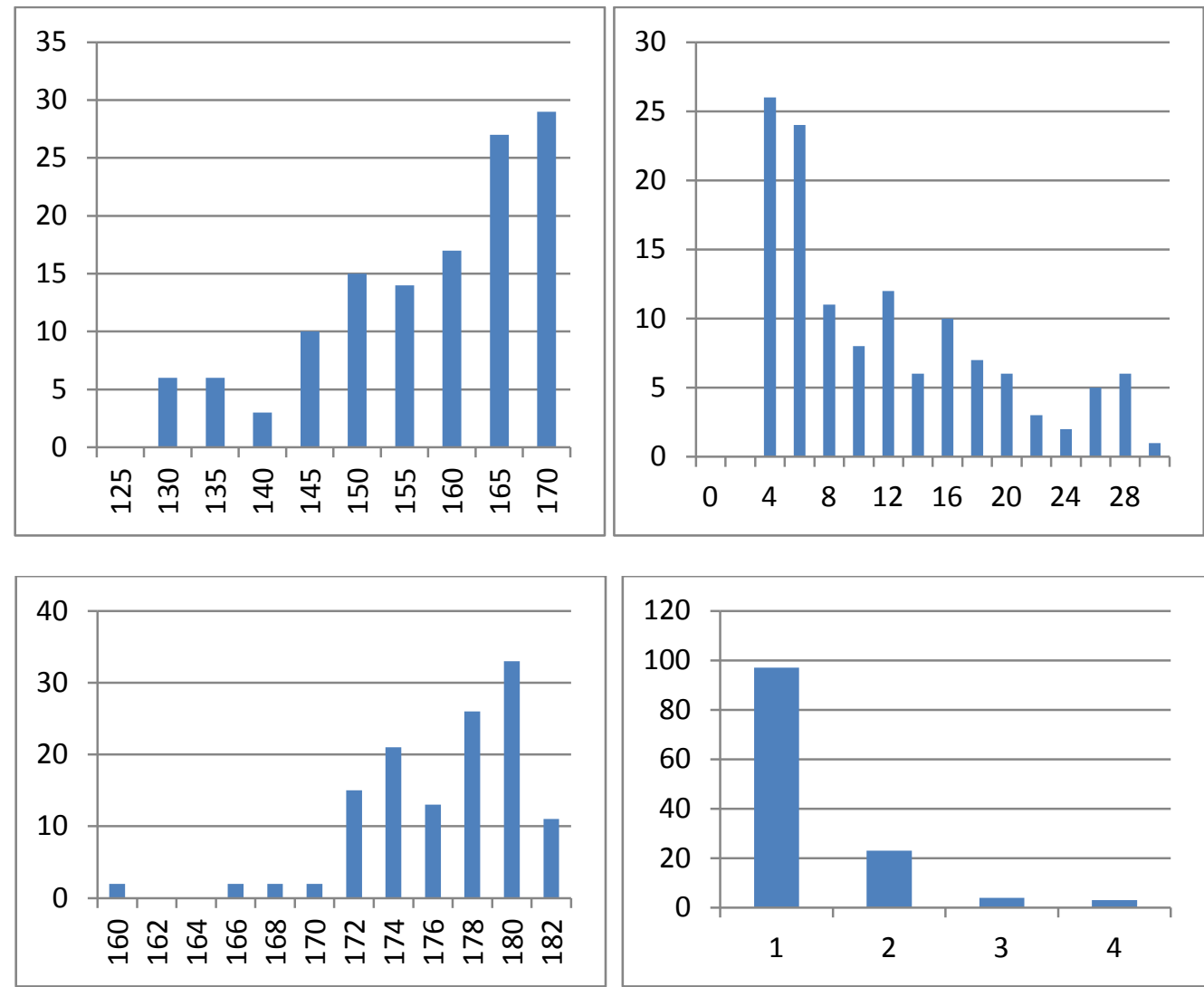

FIGURE 40. (TOP LEFT) TERMINAL (TOP RIGHT) INTERMEDIATE (BOTTOM LEFT) PATH LENGTHS AND (BOTTOM RIGHT) ORDER FOR HIGHER C4

By increasing this parameter we are increasing the rate at which bound MAP-2 is altered to become phosphorylated MAP-2. As expected the branching order was lowered by approximately twenty percent. The intermediate segments have halved in length compared to the intermediate segments of the default data model The terminal segments have increased in length by twenty percent of the terminal segments in the default data model. The lower branching order and the greatly lowered intermediate length are due to the shortened branching phase. By switching more phosphorylated MAP-2 to bound MAP-2 right from the start of the simulation, rather than when the transition point between branching and elongation is reached, the transition between branching to elongation starts much sooner and results in the shorter, weaker branching phase. 


\begin{tabular}{|l|l|}
\hline Morphological variable & Simulation data \\
\hline Average Terminal Length & $154.76+/-11.73$ \\
\hline Average Intermediate Length & $10.36+/-7.51$ \\
\hline Path Length & $175.46+/-4.15$ \\
\hline Order & $1.31+/-0.65$ \\
\hline
\end{tabular}

Table 44. Statistical data for higher c4 tree.

\subsubsection{Lower c4}

By decreasing c4 we are decreasing the amount of phosphorylated MAP-2 that gets altered into bound MAP-2. By lowering this, we should delay the rate of change when the F and G functions are aligned, and lengthen the amount of time the branching phase has. It is likely the overall growth of the tree will be shorter and that there is a higher branching order. Table 45 shows the values when we set the value of c4 lower than the default.

\begin{tabular}{|l|l|}
\hline Parameter & Value \\
\hline c4 & 0.5 \\
\hline
\end{tabular}

Table 45. The lower c4 
The histograms in figure 41 show the differences when c4 is set to a lower value.
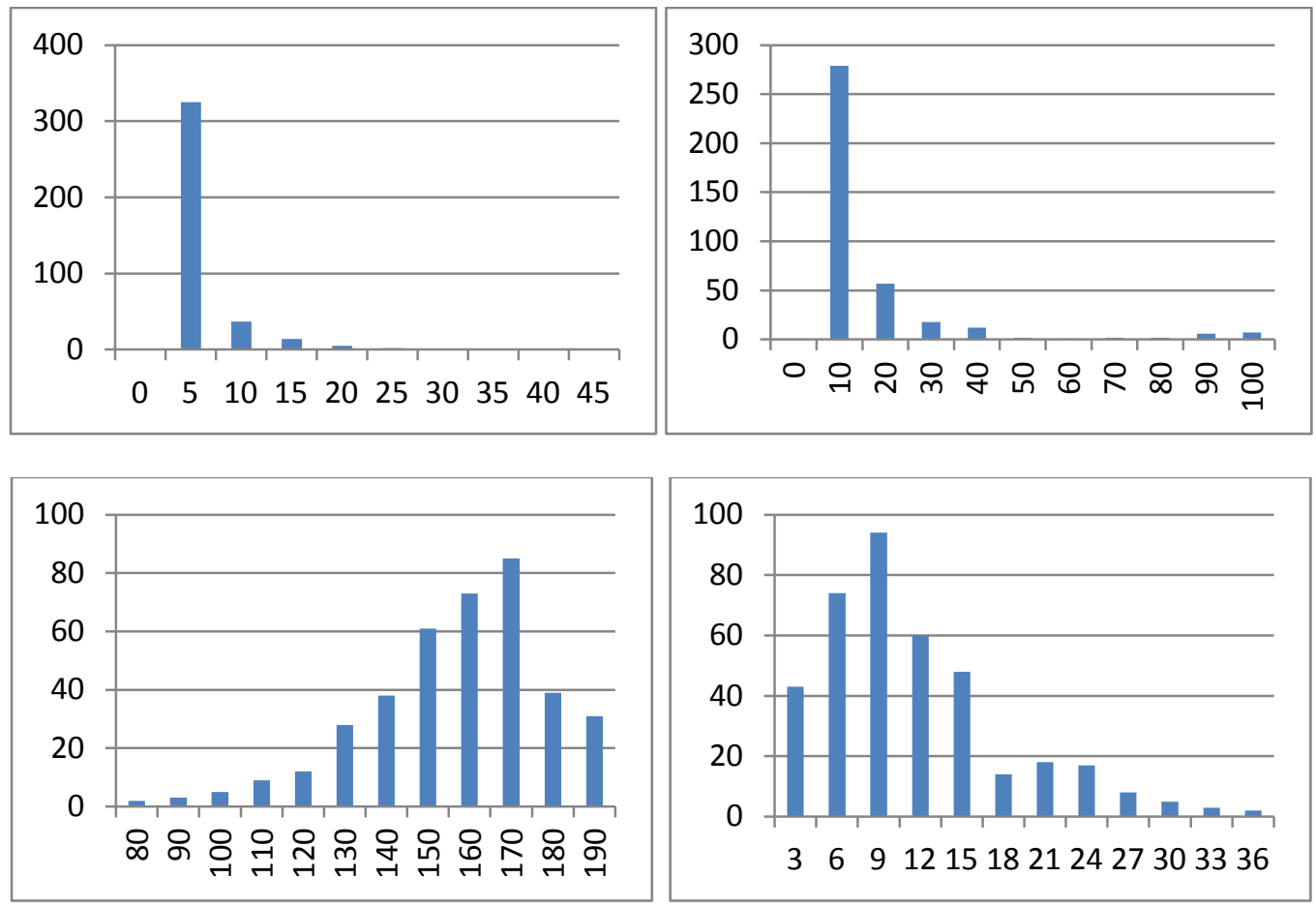

FIGURE 41. (TOP LEFT) TERMINAL (TOP RIGHT) INTERMEDIATE (BOTTOM LEFT) PATH LENGTHS AND (BOTTOM RIGHT) ORDER FOR LOWER C4

By decreasing this parameter we are decreasing the rate at which phosphorylated MAP-2 is altered to become bound MAP-2. As expected there is a seven fold increase in the branching order compared to the default model's data set. The intermediate segments are half as short as the intermediate segments in the default data model. The terminal segments are only two percent of the terminal segments in the default data set. This is due to the branching phase of the simulation lasting the whole length of the lifetime of the simulation. The F and G function's alignment is flattened by the high amount of bound MAP-2 that becomes phosphorylated MAP2, meaning that the bound MAP-2 never dominates enough to produce a very low branching probability. As the elongation of the terminal segments stalls due to a lack of bound MAP-2 and reaches a steady state in length, it continues to branch with little elongation of these branches and so producing the very short terminal segments. It bears a great resemblance to increasing c3 in its behaviour. 


\begin{tabular}{|l|l|}
\hline Morphological variable & Simulation data \\
\hline Average Terminal Length & $3.06+/-4.44$ \\
\hline Average Intermediate Length & $11.58+/-17.63$ \\
\hline Path Length & $151.73+/-21.34$ \\
\hline Order & $10.4+/-6.7$ \\
\hline
\end{tabular}

Table 46. Statistical data for lower c4 tree.

\subsubsection{Overall impact of changing the c constants}

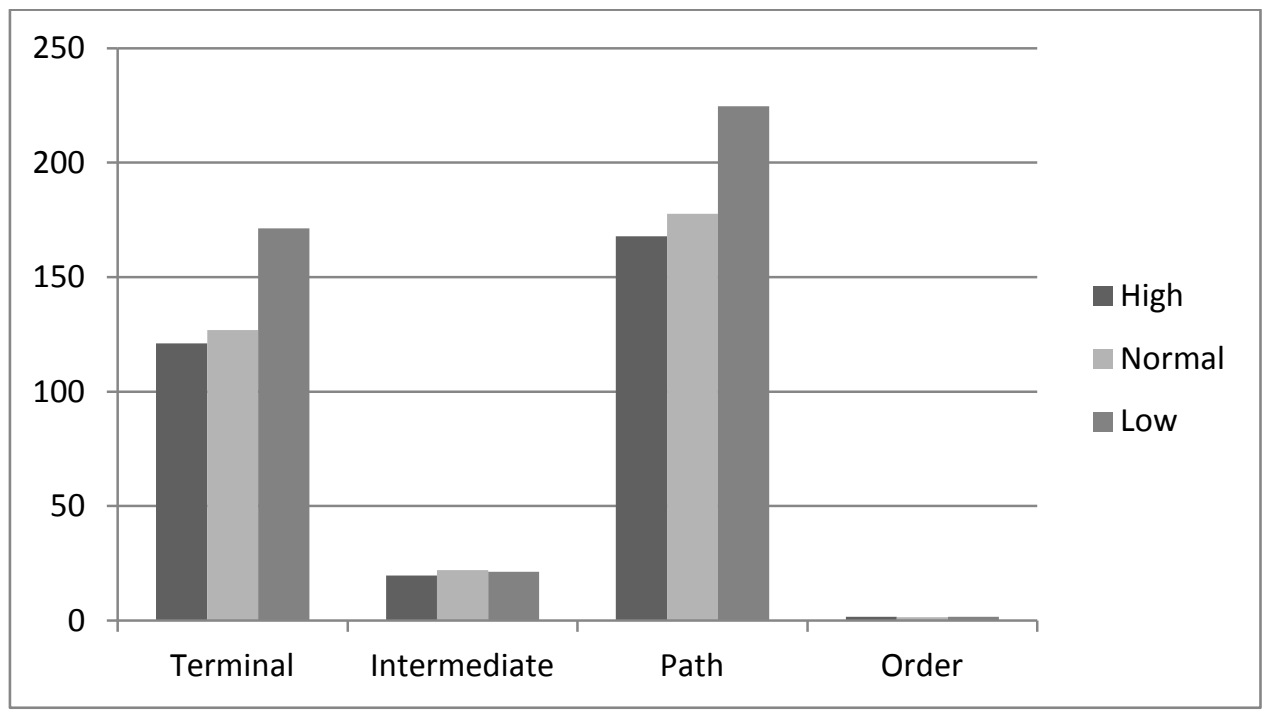

FIGURE 42. OVERALL IMPACT OF CHANGING C1

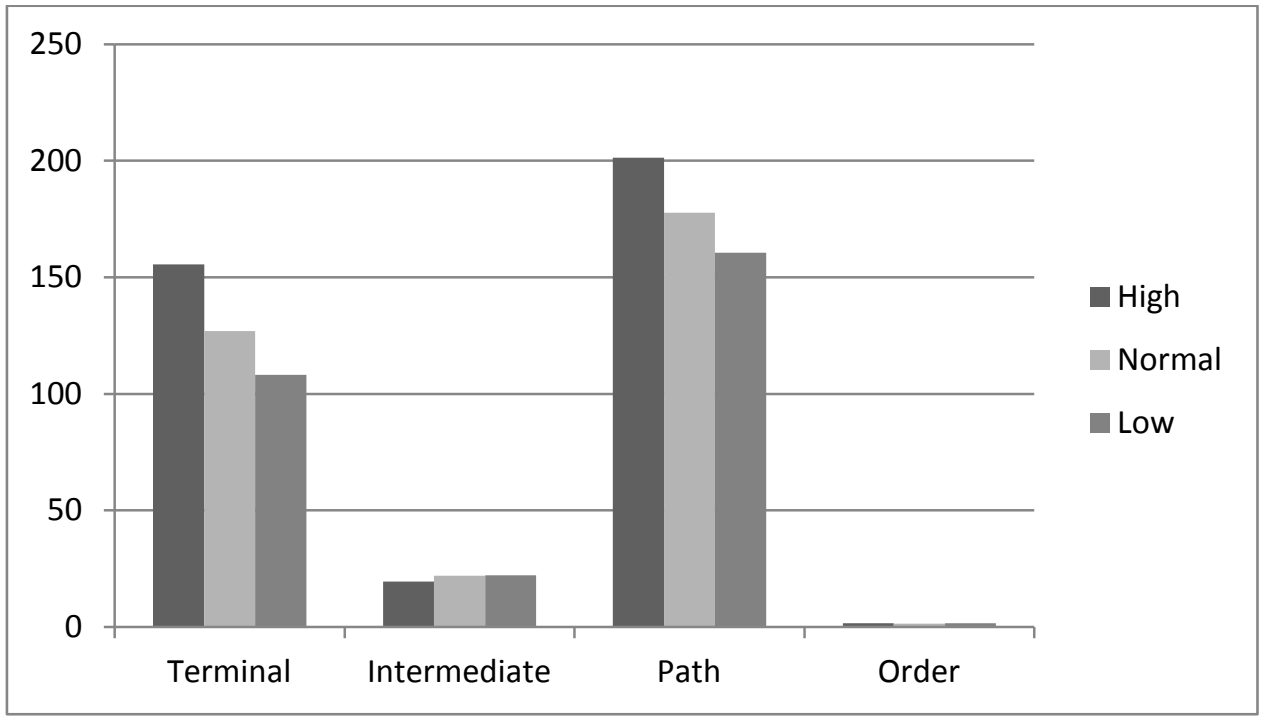

FIGURE 43. OVERALL IMPACT OF CHANGING C2 
Changing c1 and c2 alters the rate at which unbound and bound MAP-2 feed into each other. As the amount of unbound MAP-2 is usually greater than the amount of bound MAP-2, changing c1 has a far greater effect on the shape of the tree than c2 does. For the most stable and predictable neurite structures, it is suggested that these constants are left roughly as they are, as shifting the $\mathrm{kF}$ and $\mathrm{kG}$ values has the more subtle effect of sliding the transition point in time and change the simulation from a branching state to an elongation state.

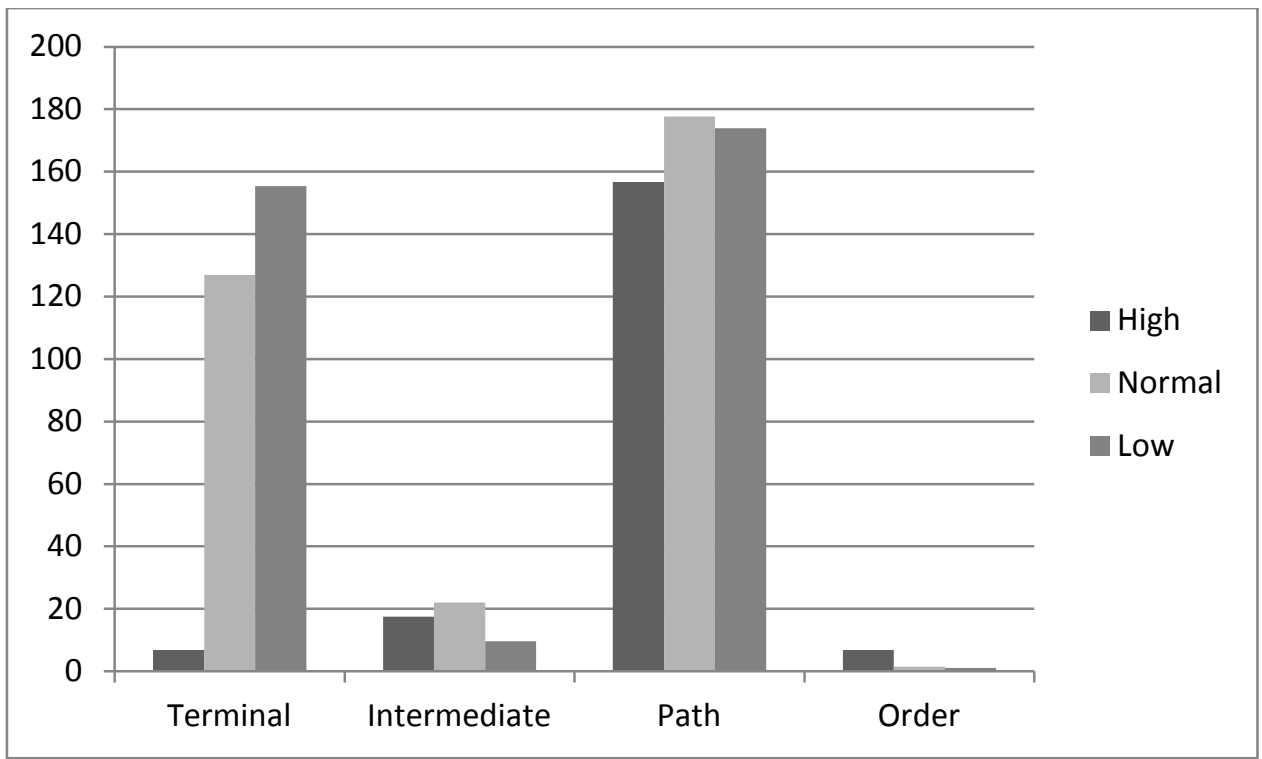

FIGURE 44. OVERALL IMPACT OF CHANGING C3

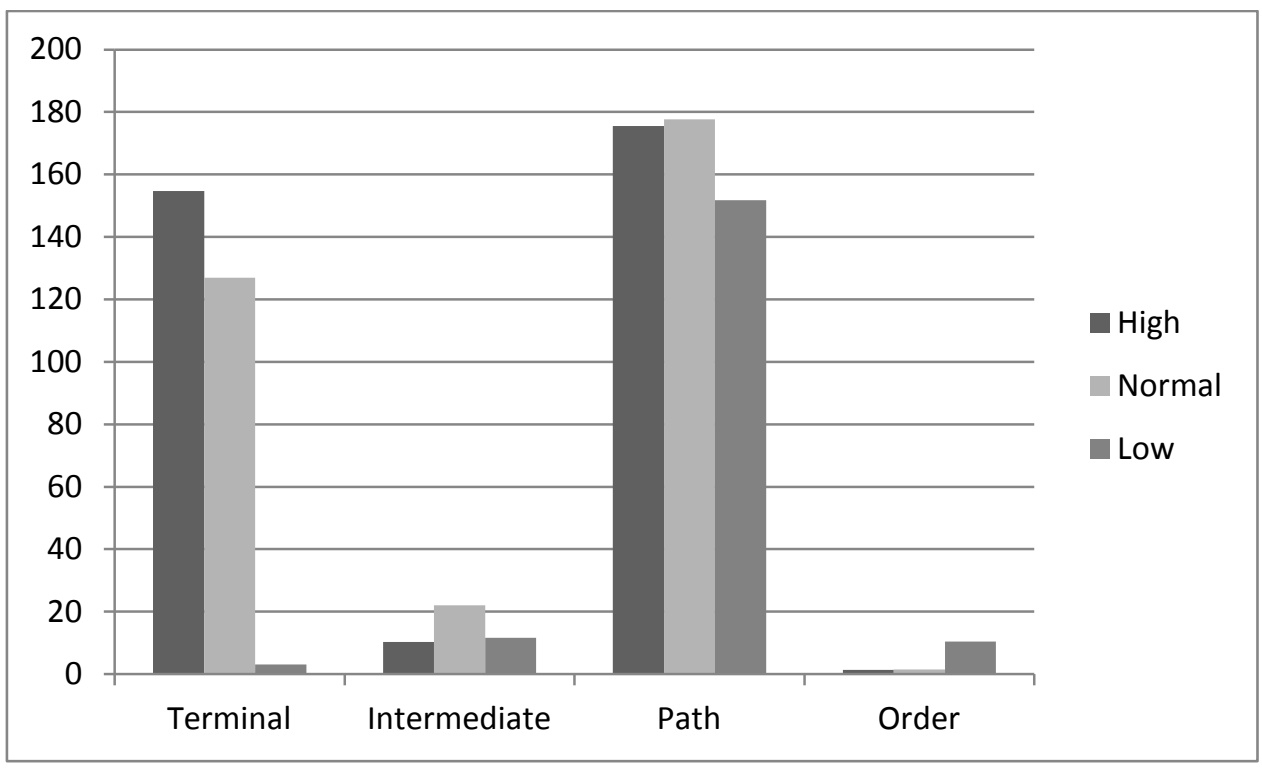

FIGURE 45. OVERALL IMPACT OF CHANGING C4 
Changing c3 and c4 alters the rate at which bound and phosphorylated MAP-2 feed into each other. This is a much more obvious process than altering c1 and c2 as the ratios between the bound MAP-2 and phosphorylated MAP-2 states is far more controllable. Altering these values provides fine grained control into the transitions between the elongation and branching states as the calcium levels approach the point of equilibrium between the $\mathrm{F}$ and $\mathrm{G}$ formulae. Altering $\mathrm{kF}$ and $\mathrm{kG}$ provides the baseline for the transition point, the c3 and c4 parameters provide the subtlety around that point.

\subsection{What are the other parameters in determining tree type?}

The parameters widely vary as to their effect upon the model. Changing the power on the rate converters provide the most fine grained control over the generation of the neuronal structure. Other factors are less key. A small amount of tubulin can produce a fair sized structure if assembled appropriately. Likewise, a large amount of free tubulin in the system may be wasted if it is not bound in large amounts. The conversion rate changes are very sensitive to change. A small alteration in the rates is propagated through repeated time steps until the end result is significant.

The decay rates for tubulin and the various MAP-2 states are important in the scheme. At the initial stages, they introduce a level of restraint into the growth and produce a more realistic method of growth, with starved branches decaying, and allowing the spread of the remaining resources to be used more appropriately. These are also very sensitive as the values in the compartments are often very small and removing a percentage of this every time step can be a significant drain. This has a detrimental effect in branches which are struggling due to resource competition. Where they would suffer Armageddon events and be subsumed back into the intermediate, they remain as they are and become resource sinks, unable to progress due to a lack of resources but pulling in valuable resources from other parts of the tree.

The calcium change rate constants are proven to have the widest and more profound effect on the morphological shape of the tree, by swapping which value is higher the model can be set up 
to initially elongate or branch and remain that way, or transition to a different state. This has a fundamental effect on the overall shape of the dendritic structure, the other parameters merely alter this overall shape by their known quantities. 


\section{CHAPTER 5}

\section{Model Analysis}

\subsection{Emergent behaviours}

There were several emergent behaviours noticed with the model.

\subsubsection{Steady state}

When outgrowing, a single neurite could reach steady state, where the factors in the branch were balanced and so the branch remained stable and unmoving. This can be seen in figure 46 and 47. Figure 46 shows the neurite over a period of time, unmoving in its length. Figure 47 shows that the values tend to oscillate slightly around a stable point rather than being exactly stable, but this is to be expected in a mathematical system such as this. In a biological system, this would likely occur as filopodia extend and retract along the growth cone, causing small fluctuations in the chemical balance which in turn would cause slight fluctuations in the concentrations of chemicals in the system.

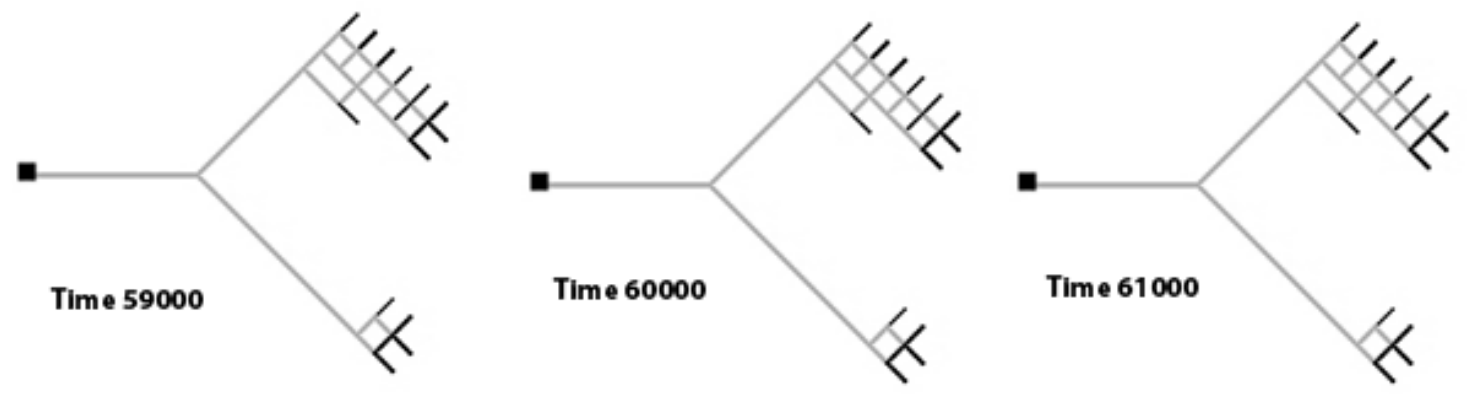

FIGURE 46. STEADY STATE SYSTEM

The entire structure can also reach a steady state. In this event, the competition for resources is not fierce and each neurite can balance its own requirements. This is an unlikely state as each branch competes for material and so the entire structure is too dynamic and changing for this to 
occur often. The main reason a steady state structure is unlikely to occur is the random nature of the branching. A truly steady state structure would have an infinitesimally small chance for a branching event to occur at each of its branch tips. A branching event upsets the delicate balance of a steady state structure and can alter the entire topology of the neurite.
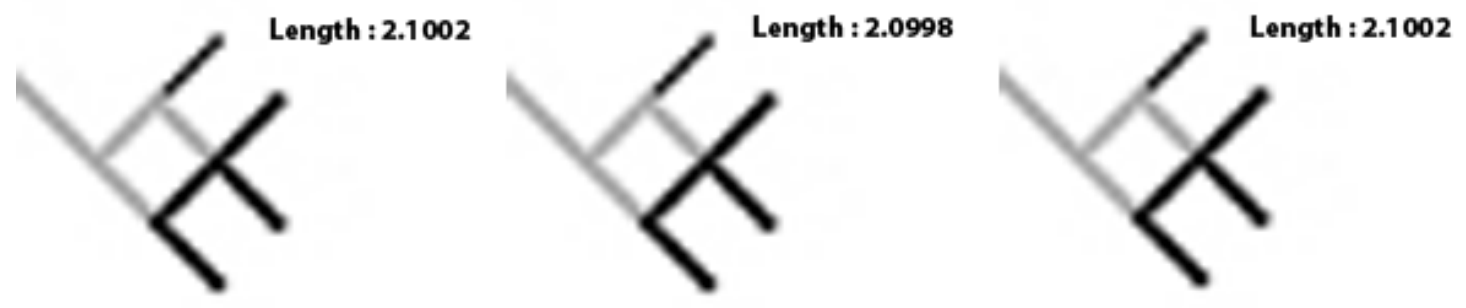

FIGURE 47. OSCILLATIONS AROUND A POINT IN THREE CONSECUTIVE TIME BINS

If competition for resources grew too great and a branch was starved of material, it would begin to retract until it either stabilised or received greater resources.

Figure 48 shows an unstable branch with retraction occurring and figure 49 shows the imbalance in resources. The branch in figure 48 has not yet suffered a component loss due to the retraction. As figure 49 (see 5.2) shows, when there is not enough free tubulin (more likely) or when the free tubulin is not being bound in sufficient quantities (less likely), a retraction of the branch can occur.
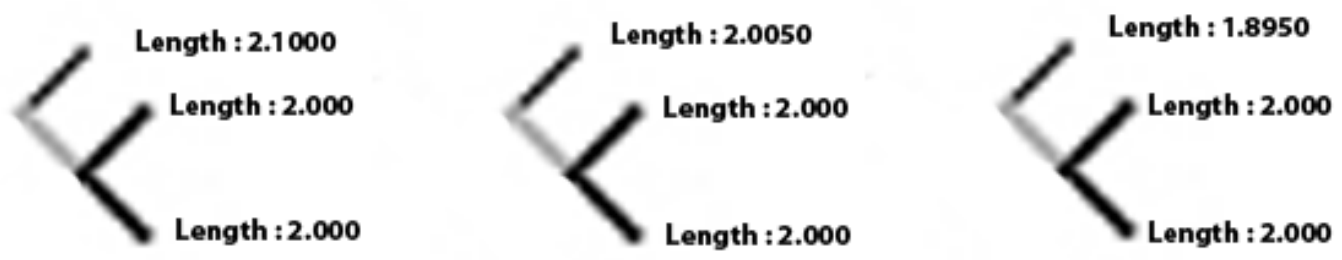

FIGURE 48. RETRACTION IN UNSTABLE BRANCH

The trailing compartment shrinks back as the microtubule assembly collapses. It is possible, in a particularly starved branch for an Armageddon event to occur, where the branch collapses entirely back into the trunk. This model didn't account for this occurrence and so always allowed for a minimum of a compartment in a branch. 


\subsubsection{Armageddon events}

Armageddon events are where a branch has too much competition for resources and cannot maintain the structure of the growth cone and terminal segments. As the tip recedes, the trailing compartment shrinks until the structure can be maintained with the available resources. If there are not enough resources to maintain the branch at all, the branch retracts into what was its sister branch, forming either a single intermediate segment or a single terminal segment and releasing the chemicals within the segment back into the tree at the branching point. In the system this would be implemented as whenever a branch dropped below the minimum length for a compartment it was removed and the chemical concentrations present in the compartment would be added into the compartment which was the root of the branch.

There are several reasons why this was not implemented in the program even though the behaviour of the system emerged and became a candidate for addition. Firstly, the point where the branch collapsed, the segment had tubulin bound into microtubules which was effectively unaccounted for by the system at the point where the segment was small enough to be removed by the system. As the system has no effective idea of how much tubulin there should be freed following the collapse of the branch, there is no means the system can use to accurately release. I attempted some approximations such as keeping track of the amount of tubulin assembled / disassembled / decayed, see the terminal tubulin equation in 3.1, but this raised the issue of the branching events taking a single compartment and splitting this into four. By making no assumptions about how the micro-tubules worked in the branching event within the system, it was impossible to accurately model the amount of bound tubulin at any one particular time. This tended to end up with too much material being added back into the system when it was implemented and caused a serious destabilising effect on the system.

This meant that a branch was left to be a minimum length even though it did not have enough resources to support it. The implementation bounded the levels to a baseline of zero, that is the branch could not become a chemical sink, sucking more chemicals out of the system than it 
would in reality. This gave the potential for further emergent behaviour as the dormant branch was given the opportunity of re-awaken as discussed below.

\subsection{Competition between branches}

Competition between branches is where there is a limited amount of resources available to the tree and each branch requires as much of the available resources as possible in order to grow. This concept was introduced in a model by Arjen van Ooyen (van Ooyen et al, 2001). Diffusion will move a larger amount of material into a compartment with less material, so that if one branch is doing particularly well and growing faster than its partner branch, it is likely to be using more resources and so the sink levels of each branch will be disparate as shown in figure 40, the relative amounts of tubulin in the unstable branching. This means that a tree which is particularly asymmetrical in terms of branches will supply more resources to the side of the tree with more branches and starve the side of the tree with fewer branches. The over supplied branch becomes dominant. The figure has the top branch starved of tubulin, as the lower branch requires more resources and so sucks more resources from the both branches shared intermediate segment.

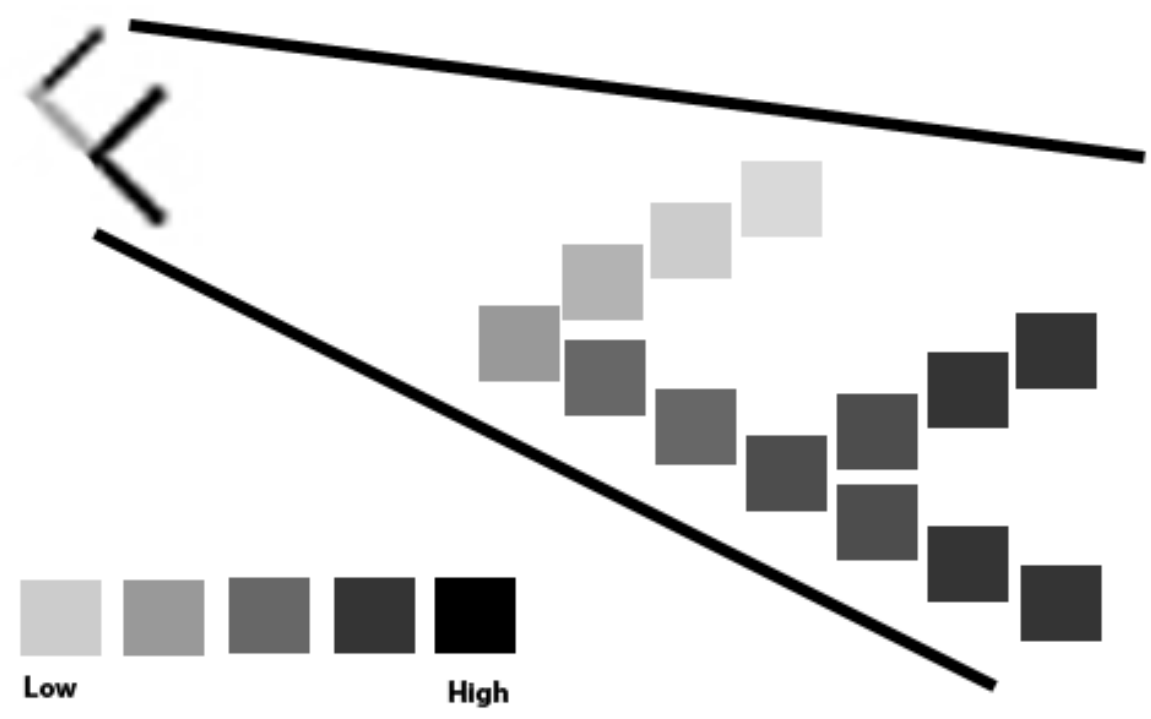

FIGURE 49. A TUBULIN STARVED BRANCH AND WELL FED BRANCH 
The result of this is that a dominant branch tends to remain dominant throughout the life of the tree unless branching events change the equilibrium between the two branches. A starved branch will remain dormant, either withering as described above, or remaining static until receiving a fresh dose of materials. This agrees with the model put forward by Arjen van Ooyen (van Ooyen et al, 2001) where they encounter similar behaviour based upon competition. The dominant section of the tree is always likely to remain dominant. The level of dominance is based on the amount of branches relative in each section of the tree, a high dominance subsection of the tree will always remain dominant, a low level dominance subsection of tree has to be balanced up with a few random branching events.

The event which changes the level of dominance is a random branching event happening to the dormant or starved branch. A branching event means the branches towards the root of the tree require more resources, emptying the preceding compartments at a greater rate. As diffusion is a much more effective means of transporting chemicals that active transport, by increasing the disparity between the root compartment and the dormant branch, more material is supplied to the dormant branch potentially changing the equilibrium between the previous dominant and dormant branches.

A dominant branch can also reach a point of equilibrium, whereby the branches reach a point where the material being fed into them equals the amount of material being consumed. This can lead to the segments further towards the root of the tree reaching an equilibrium also which can mean that there is material left over for the competing branch. This is an infrequent occurrence however as the dominant branch needs to have a low dominance level in order for this to make a difference. The general behaviour of the model is for high levels of dominance.

So there are two main ways in which a branch can move from being dormant into becoming active again, either by the competing branch reaching a state of equilibrium and so freeing up resources for the dormant branch to use, or by the dormant branch having a branching event which raises the amount of materials required by the dormant branch and creating a resource sink that then gets filled by the rest of the tree. 


\subsection{Variants}

By removing certain elements of the model interesting comparisons can be made. It is expected that the same behaviours would be found in the reduced models as are found in the full model. There are two ways in which the model can be easily altered. By removing branching from the model so that the neurite elongates only, and to remove the randomness of the branching events so that they are regular within the system.

\subsubsection{Elongation}

Predictably, the elongation variant, without any branching, grew one of three ways as described below.

In fig 50(right), the amount of free tubulin and the amount of bound MAP-2 is very high. The result in this is that the neurite extends effectively until the end of the model's allotted time. The assembly rate so far outstrips the disassembly rate that the growth is unlikely to stop within the time frame of the simulation. The trees produced by the standard model which do not branch are very similar to this, as they extend with high level of free tubulin and bound MAP-2, and as time goes on, they are more likely to resemble this variant as the chance of branching approaches zero.

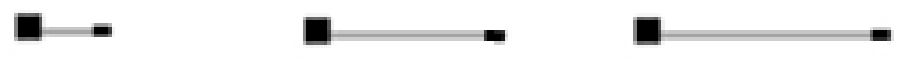

FIGURE 50. (LEFT) LITTLE TUBULIN, (CENTER) STEADY STATE, (RIGHT) CONSTANT ELONGATION

In fig 50(left), the amount of free tubulin and the amount of bound MAP-2 is very low. The model appears to have created a steady state, but as can be seen, the value of the disassembly rate is much higher than the assembly rate. The neurite would collapse if the model allowed it. This shows that there is very little growth, if at all any under these circumstances. 
Fig 50(centre) is the hardest to produce. The balancing act in the model of the free tubulin and the amount of bound MAP-2 results in an amount of initial growth and then stability. As there are no branching events to disrupt this stability, it will remain in this state until the model ends. It is virtually impossible to produce without a certain amount of cheating in the model. As calcium continues to grow, all the MAP-2 being produced is being turned into its bound state, which along with the free tubulin, must exactly equal the disassembly rate. The easiest way to create this effect in the model is to up the decay rates of the various MAP-2 states and the disassembly rate until a steady state is found.

These variants show stable states and retraction to an eventual collapse if they were allowed. Competition could not be examined as there was only a single terminal segment in the neurite.

\subsubsection{Elongation with fixed branching rate}

This was more unrealistic than the random branching. A fixed branching rate produced a tree as shown in figure 51. In figure 51(A) with a low static branching rate, the initial trunk is long until it has reached a steady state. After this has branched, there is not enough material to sufficiently grow beyond the initial compartments so the tree results in a dandelion like arrangement. A long trunk with a shock of short branches at the end. This showed all the behaviours that would be expected of the model. 
(A)
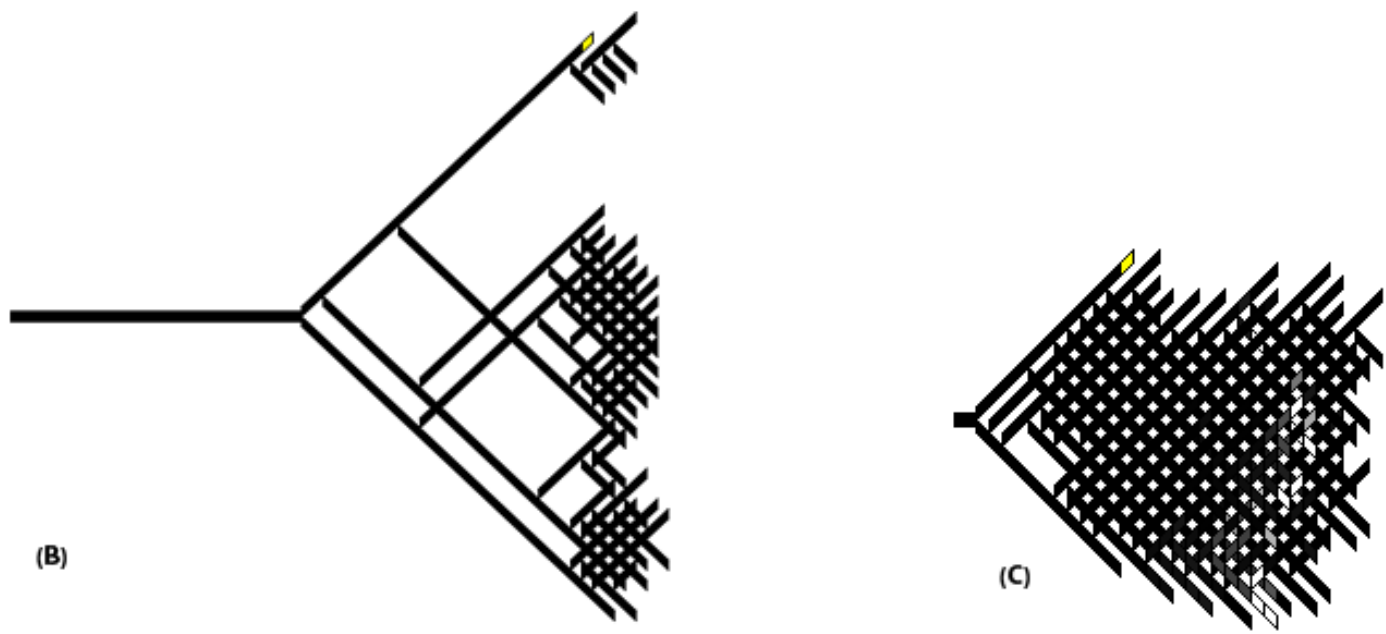

FIGURE 51. ELONGATION WITH FIXED BRANCHING

In figure 51(B) the branching rate is typical for the results for the default parameter values shown in chapter 4 . It produces a very asymmetric structure as the first side to branch an additional time gains much of the resources and will always be larger and have more branches than the weaker branch. The structure ends up rather short and much like the dandelion structure on one side, and a much longer and fuller tree on the other side. Statistically, this will appear to be relatively similar to the full model, but this is due to the law of averages rather than consistency. This again shows the behaviours apparent in the fuller model.

In figure $51(\mathrm{C})$ the branching rate is high. It produces a very short tree. As it branches repeatedly in a short space of time, the resources are directed into making the tree flourish as widely as possible rather than extend the branches. In a model where apocalyptic events were possible, a large percentage of the branches would fail due to a lack of resources and collapse, allowing greater resources for the remaining branches. However in practice, the branching rate would keep up with the destruction of branches and maintain the poor structure. Over time, this 
tree would appear to be a waving mass of short tentacles, waving and undulating. In this model, there are no apocalyptic events, so the amount of resources in the system continues to fall as the branching continues, making the hatching effect of many branches crossing repeatedly in the two dimensional representation of a three dimensional space.

These variants show the full range of behaviours expected from the fuller model, but their impact upon how the topology and structure changes is muted by the effects of fixed branching rates. Branching is often the dynamic which can reinvigorate a branch during competition or reduce the levels of resources in a branch so that it starts to retract. These branching events in the full model are affected by the state the structure is in. A starving branch may have a high chance of branching, which may start the recovery process. Retraction often concentrates what little resources a branch has to contribute to this effect. By removing the randomness of the branching, the full potential of these behaviours is lost.

\subsection{Limitations of the model}

\subsubsection{Retraction}

The model does not cover retraction particularly well, as it always needs to retain a certain amount of compartments to allow a terminal segment and the growth cone compartment right behind it. This means that the minimum length for a branch is $2 * d x$. In order to maintain this structure I turned off retraction in the model entirely. While retraction could be recorded in order to show that stable states could be achieved, the retraction was not applied to the segment. With the retraction in place a stagnant branch could retract all the way to the two initial compartments and not further, which was ridiculous. This scenario did not handle the concentrations correctly and so an attempt at adding full collapse of branches was added. This did not work terribly well as partially outlined above, the minimum two compartments had to stay, so a variety of options around the collapse were attempted. 
The first attempt recorded the amount of negative length the compartments possessed and once the negative growth reached $2 * d x$, I removed the two compartments and moved the concentrations into the intermediate compartment which was previously the branching compartment. This messed the concentrations of the chemicals up as the length was constant as far the model was concerned but the real length was much shorter. Actually shortening the growth cone compartment until it reached zero length was also a mistake as the concentrations grew ever higher due to the reduced volume and started growing again, until the volume reached a sufficient volume as to commence shrinking again, and so on providing little solution.

This is the most important thing the model is missing, without it certain tree types such as symmetrical trees, are difficult to produce, and the true development of the neurite is not biologically plausible without it. The dendrite should be more of a dynamic living entity than the current model makes it, without the constant extrusion and collapse of branches until one fully develops or stabilises, a snapshot could not be taken at any point in time before the full development life of the neurite and accurately say that the simulation was correct at that point.

\subsubsection{Stabilisation of branches}

Despite the model's ability to come to a steady state, it does not cover the stabilisation of branches. Over the course of my research into how the development of neurites occurred, there were several mentions of events which stabilised specific branches such as synapse creation (Cline, 2001). The model would be more biologically plausible if there was also a random chance of branches stabilising at their current length. The act of stabilising a branch would stop elongation and prevent branching from the terminal segment, it would also reduce the amount of resources required for that branch, allowing it to effectively become dormant at its current length. This would provide a boost for the rest of the tree which would then have more resources with which to work. The stabilisation point would be a third state in the calcium 
enabled transitions, with a terminal moving from a branching state to an elongation state, and eventually into a stable state.

This would provide yet more fine control over how the morphologies of the trees could be generated, as at the moment, if the time assigned to the simulation is long enough, certain sets of parameters can grow a tree practically indefinitely, which is obviously not biologically plausible. The steady state outlined above is not a sufficient replacement for this, as a single random branching event can disrupt the steady state and send the whole tree into another round of branching or elongation. A steady state is not the same a stabilisation.

\subsubsection{Interstitial branching}

The model does not allow interstitial branching, focusing only on the terminal segments and their growth cones. This stops the model from being biologically plausible as many more mature neuron types have interstitial branching as being one of their main features. This again prevents a snap shot of the developing neurite being taken at any moment in time and accurately say that the simulation was morphologically accurate against a developing neurite in vivo.

The model would be changed so that it includes concentrations of MAP-2 in all three states in all the compartments in the simulation. Unbound would continue to act as it currently does, bound MAP-2 would have little effect before the branching event, and phosphorylated MAP-2 would destabilise the intermediate compartment it was in. Given a particular random chance, and this could initially be the same formula as the branching probabilities in the terminal compartment, a branch could spontaneously form, assuming there is a sufficient level of free tubulin and bound MAP-2 to allow a certain percentage of the new compartments to form. If there isn't enough tubulin or MAP-2 to form the new branch, ignore the branching event. If the retraction worked properly in the model, the compartments could be formed anyway and allow retraction to deal with them if it was appropriate. 


\subsubsection{Asymmetric trees}

The model tends to produce asymmetric trees which stops the model from being biologically plausible as most neurons are symmetric in nature. The model frequently grows a tree with a lopsided initial branch, with one side of the branch containing few subbranches, and the other side being fully grown. If there was properly modelled retraction of starved branches, and the stopping of growth in fuller branches due to stabilisation, the model would produce more symmetrical trees. 


\section{CHAPTER 6}

\section{Conclusions and further work}

\subsection{Conclusions}

The main aim of this thesis was to continue bridging the gap between statistical models and biologically plausible models of neurite branching and outgrowth. It was to build upon the works of Hely, Graham, and van Ooyen (Hely et al, 2001) by adding additional features to these works, namely by adding tubulin concentrations into the mathematical model and ensuring that elongation was dependent upon it based upon the levels of bound MAP-2. Diameter changes were introduced to the simulation where by the trunk of the structure grew rather than shrinking the branches. A wide variety of trees was produced, and it was shown that by altering the constants within the model, different statistical results were produced.

A model was constructed which included the elements of work from previous researchers. These initial models were then altered to become more plausible in terms of the biological aspects of the model. Tubulin production and transportation was modelled and the elongation functions were altered to become dependent on the amount of tubulin in the simulation along with the amount of dephosphorylated MAP-2. The elongation of the model became an approximation of the microtubule process which a real neurite is dependent upon for its growth during development.

Diameter changes were introduced where the trunk of the structure grew, rather than the branches shrinking as in previous research (Hely et al, 2001). This promoted other factors other than diffusion such as the active transport of tubulin and the influx of calcium along the length of the neurite.

A wide variety of trees were produced. It was shown that the main three types of trees, short terminal segments with high amounts of branching, long terminal segments with few branches, and intermediate and terminal segments of equal length with an average amount of branching 
could be produced. These could be produced by controlling the point at which branching within the model switched to becoming elongation using the calcium rate conversion parameters. The $\mathrm{kF}$ and $\mathrm{kG}$ parameters were the key to these trees as they controlled this switching point. In a simulation where the spread of calcium was likely to begin at zero and raise to around three hundred by the end of the simulation having the parameters set to $\mathrm{kF}=0$ and $\mathrm{kG}=10$ producing a tree with a short branching phase and a much longer elongation phase. At the start, while calcium is below $10, \mathrm{kF}$ ensures the $\mathrm{F}$ function is 1 , while $\mathrm{kG}$ ensures the $\mathrm{G}$ function is 0.1 at this initially low level of calcium. This pushes more bound MAP-2 into the phosphorylated state, limited by the large disparity between c3 and c4. As calcium increases the $\mathrm{G}$ function smoothly increases. When calcium reaches 10 in the terminals, the F function is still 1 , and the $G$ function has reached 0.5 . At this point the swing between phosphorylated and bound MAP-2 has already begun thanks to the effects of c3 and c4. After this point, the swing becomes greater, altering practically all the phosphorylated into bound MAP-2. It can be shown then, in this scenario, the branching order can be increased and the terminal lengths reduced until they are of a similar length to the intermediates purely by increasing the $\mathrm{kG}$ constant. By swapping $\mathrm{kF}$ and $\mathrm{kG}$, we can produce trees which start off by elongating a long distance before producing a dandelion like head of branches. In that tree, by moving the $\mathrm{kF}$ value higher and lower, we can have a longer initial intermediate segment and a lower branching order.

It was shown that the model's parameters could be altered in an understandable and consistent manner and produced expected results when tested. By altering the parameters, various morphological characteristics could be finely controlled, and given a combination of these parameter changes, a large number of possible outcomes for the final morphology could be produced.

\subsection{Further experiments}

There are further experiments that could help test the model's outcomes. 


\subsubsection{Branch competition}

An experiment designed to examine the levels of chemical concentrations during the development on the neurite to determine whether branches do indeed become starved of resources during development. For an accurate measurement, the actual concentrations would have to be measured, rather than relying on the expression of markers, while leaving the neurite intact, which is a technique researchers have not fully developed yet. A further element of this experiment, and one that would require the neurite to remain intact, would be to check that the starved branch could indeed re-establish itself and promote elongation again once the resource sink that was causing the branch to be starved in the first place was stabilised somewhat. This would require both visual monitoring of the neurite to determine at which point the growth of a branch had stopped, repeated measuring of the base of the starved branch to track the concentrations in that point over time along with the branch which is doing the starving. This would be a highly complex experiment, far removed from the typical "knockout" style experiments.

\subsubsection{Constant discovery}

An experiment to deduce the correct values for some of the constants which govern the model's behaviour. The elongation formula relies on the product of the concentration of tubulin, the concentration of bound MAP-2 and an assembly constant. By measuring the amounts of free tubulin, and bound MAP-2 in the growth cone over a period of time and determining how much elongation had occurred in that time period, an estimation of the assembly rate could be made. Likewise, in a branch which is retracting due to a lack of tubulin or bound MAP-2, measuring the values over a period of time and determining how much the branch had retracted over that period would give an estimation for the disassembly constant.

Similar experiments could produce estimations for other constants in the model, measuring the rate that MAP-2 is altered between its three states would give an estimation for the values for 
the calcium rate constants, and it would be a validation of the model if the estimations for these constants were different for different morphological types of neurons.

\subsection{Further work}

There are several areas that can be used to extend this model further and provide not only a more biologically plausible model, but also a more accurate one.

\subsubsection{External environment}

Modelling the external environment would open up several avenues of further work. It would allow a proper three dimensional environment for the dendrites to grow into, it would be able to provide cues for the dendrites to respond to. Excitatory factors such as additional sources of calcium and inhibitory factors such as the boundaries of a substrate would be added to allow further control over how the dendrite grows and the morphology it would take on after the development had been completed. Providing a "playing field" for the dendrite to grow in would allow accurate modelling of the effects these guidance cues have upon the morphology. These cues would be randomly placed and of varying strengths within a plausible framework, it is unlikely there would be a strong inhibitory source very close to the soma for example. This would be hard to match up to a statistical model at this point as few of the statistical models take into account external factors such as this. Further neurons come be placed into this "playing field" to allow networks to form, combined with the synapse work, the could form fully fledged neural networks and open up a whole new avenue of research for this model, modelling not only the development of a single neurite but the development of networks of neurons. 


\subsubsection{Growth cone}

Modelling the growth cone would require the external environment work to be completed. It would follow guidance cues from the environment providing pressures on the growth cone which would affect the chances of branching and the amount of elongation performed. In a three dimensional environment it would allow the growth cone to properly turn to follow cues and so would be able to produce another traceable statistic, that of branching angle. Branching would be within the correct angular range for the neuronal form that was being created. It would also allow proper volume calculations to be tracked for dendrite dispersal. The cues acting on the growth cone would provide tension and increase the chances for branching to occur, or to inhibit growth entirely and allow the growth cone to collapse and have the branch destabilise and retract. This would act as the basis for further modelling of the growth cone elements.

\subsubsection{Actin assembly}

The actin assembly would not necessarily require any other pieces of extension work to be completed, though it would benefit from having the growth cone work available along with the external environment. The actin assembly would further model the growth cone turning the filopodia into proper actin microfilaments. The growth cone would be deformable and elastic, the filopodia expanding on the growth cone work by providing stress through adhesion. The chances for branching would be affected by these stresses but also by the strength of the actin, weaker or thinner actin in the growth cone leads to a greater chance of branching if placed under stress. The growth cone as it elongates leaves the actin sheath elongating behind it, and so this could also be modelled which would allow for synapse formation and interstitial branching to be accurately modelled. The sheath coating the dendrite that is not growth cone would have no further effect on the branching or elongation chances of the growth cone. 


\subsubsection{Synapses and spines}

Synapse formation would take into account two pieces of work and would rely on the actin assembly work and external environment work being completed. Synapse formation would allow spines to grow on the actin sheath due to environmental cues and would allow synapses to form there with a random regularity and strength. This would provide another piece of data to track and correlate in the frequency and density of spines created. At a random point in the development the neurite's synapses would start receiving action potentials which would propagate and allow for an additional influx of calcium into the dendrite with all the effects that this has upon the development of the neurite. The spines would also be correctly unstable and so would extrude and collapse as in experimentally observed cells. A further piece of work once this was completed would be to model the voltage gated calcium channels themselves for even greater accuracy.

\subsubsection{Modelling microtubules}

This would include two pieces of work and would not be dependent upon any other piece of work being completed. The microtubules would be properly constructed by tracking the amount of tubulin through the tree and by assembling each microtubule and by modelling the amount of microtubules in the bundle along with their cross linked strength. When retraction occurs, tubulin would be properly freed up by the microtubules and tracked, and retraction could be properly performed. Once a microtubule bundle had retreated back to the point of the branching point, the growth cone would be destabilised and could suffer a chance each time bin to collapse also. This would have an effect on how both the branching probabilities were calculated and the elongation rate was calculated. They would both ultimately be a function of MAP-2, but these would be more accurately tracked and there would be a closer linkage between tubulin bound in the system to the actual length 


\subsubsection{Interstitial branching}

Interstitial branching is where established dendritic intermediate segments go through branch creation. This starts with an environmental cue so would require the external environment work to be completed before this extension. Ideally the microtubules and actin assembly work would be completed also so that this could have the correct destabilising effect on the actin sheath and the microtubules could be correctly destabilised. The environmental cues could be varied in terms of regularity and strength to encourage particular neuronal morphologies. These would develop differently to the ones discussed in this thesis. The initial intermediate segment would elongate quickly to much of the dendrite's full length before branching, interstitial branches would then form off this main trunk and act in a similar fashion by elongating strongly initially then forming interstitial branches, while the tip of the trunk branched normally. This would produce morphologies that looked incorrect initially, but would display the correct morphology once completed.

\subsubsection{Diameter changes}

The diameter change of the simulation has been set to a power law of 3/2. In other words, the diameter of the trunk of the branching segment gets set to 1.5 times the length of the largest of the two branches. This value had no testing in the course of this work, and further work to vary this value and record the differences this makes to the model would be useful. Finding out the real values of thickening from real trees, and whether these values are different for different tree type, then adding these factors into the model would make it more biologically plausible. 


\section{Bibliography}

Abe, K., Chisaka, O., Van Roy, F., Takeichi, M. (2004) Stability of dendritic spines and synaptic contacts is controlled by *N-catenin. Nature Neuroscience 7:4 37-363

Acebes, A. and Ferrus, A. (2000) Cellular and molecular features of axon collaterals and dendrites. TINS, 23: 557-565.

Addcock, K.H., Metzger, F., Kapfhammer, J.P. (2004) Purkinke Cell Dendritic Tree Development in the Absence of Excitatory Neurotransmission and of Brain-derived Neurotrophic Factor in Organotypic Slice Cultures. Neuroscience 127 137-145

Aeschlimann, M. (2000) Biophysical models of axonal path finding. PhD thesis, University of Lausanne, Faculty of Science, Switzerland.

Ascoli, G. (2002) Neuroanatomical algorithms for dendritic modelling. Network: Comput. Neural Syst., 13: 247-260.

Ascoli, G., Krichmar, Jeffrey L. (2000) L-Neuron: A modelling Tool for the Efficient Generation and Parsimonious Description of Dendritic Morphology.Neurocomputing

Audesirk, G., Cabell, L., and Kern, M.. (1997) Modulation of neurite branching by protein phosphorylation in cultured rat hippocampal neurons. Developmental Brain Research, 102:247260. 
Baas, P.W., Buster, D.W (2004) Slow Axonal Transport and the Genesis of Neuronal Morphology. Journal of Neurobiol 58: 3-17

Berry, M Bradley, P.M. (1976) The application of network analysis to the study of branching patterns of large dendritic fields. Brain Research 109 111-132

Bhatt, D., Zhang, S., Gan, W. B. (2009). Dendritic Spine Dynamics. 71. 261-282

Bonner, J., O'Connor, T.P. (2001) The Permissive Cue Laminin Is Essential for Growth Cone Turning In Vivo. The Journal of Neuroscience 21 (24) 9782-9791

Boyd, J.P. (1989) Chebyshev and Fourier Spectral Methods. New York: Springer-Verlag. Burnett DS (1987). Finite Element Analysis: From Concepts to Applications. Reading, MA: Addison-Wesley.

Burke, R.E., Marks, W.B., Ulfhake, B. (1992) A Parsimonious Description of Motorneuron Dendritic Morphology Using Computer Simulation. The Journal of Neuroscience 12 (6) :24032416

Canuto, C., Hussaini, M.Y., Quarteroni, A. and Zhang, T.A. (1988) Spectral Methods in Fluid Dynamics. New York: Springer Verlag.

Carriquiry, A.L, Ireland, W.P, Kliemann, W., Uemura, E. (1991) Statistical Evaluation of Dendritic Growth Models. Bulletin of Mathematical Biology 53:4 579-589 
Cline, H. (2001) Dendritic arbor development and synaptogenesis. Current opinion in neurobiology. 11:118-126.

Cuntz et al. (2007) Theoretical Biology and Medical Modelling 2007 4:21 doi:10.1186/1742$4682-4-21$

Cuntz, H., Forstner, F., Borst, A., et al. (2010) One rule to grow them all: a general theory of neuronal branching and its practical application. PLoS Computational Biology 6 (8).

Dickson, B.J. (2001) Rho GTPases in growth cone guidance. Current Opinion in Neurobiology 11: $103-110$

Dehmelt, L., and Halpain, S. (2004) Actin and microtubules in neurite initiation: are MAPs the missing link? J. Neurobiol. 58:18-33.

Dityatev, A.E., Chmykhova, N.M., Studer, L., Karamian,O.A., Kozhanov, V.M., Clamann, H.P. (1995) Comparison of the Topology and Growth Rules of Motorneuronal Dendrites. The Journal of Comparative Neurology 363: 505-516

Dotti, C. G., and Banker, G. A. (1987) Experimentally induced alterations in the polarity of developing neurons. Nature 330: 254-256.

Dotti, C.G., Sullivan, C.A. and Banker, G.A. (1988) The establishment of polarity by hippocampal neurons in culture. J. Neurosci. 8:1454-1468. 
Eberhard, J.P., Wanner, A., Wittum, G., (2006) NeuGen: A tool for the generation of realistic morphology of cortical neurons and neural networks in 3D, Neurocomputing, 70:1-3:327-342

Flanagan, L.A., Ju, Y., Marg, B., Osterfield, M. Janmey, P.A. (2003) Neurite branching on deformable substrates. Developmental Neuroscience 13:18 2411-2415

Friedrich, P., and Aszódi, A. (1991) MAP2: a sensitive cross-linker and adjustable spacer in dendritic architecture. FEBS, 295:5-9.

Goldberg, D. J., and Grabham, P. W. (1999) Braking news: calcium in the growth cone. Neuron 22: 423-425.

Goodhill, G.J. (2003) A theoretical model of axon guidance by the robo code. Neur. Comput. 15: 549-564.

Goslin, K., and Banker, G. (1989). Experimental observations on the development of polarity by hippocampal neurons in culture. J. Cell Biol. 108: 1507-1516.

Graham, B., Hely, T., and van Ooyen, A. (1998) An internal signalling model of the dendritic branching process. Euro. J. Neurosci., 10(Supplement 10):274.

Graham, B., and van Ooyen, A. (2001) Compartmental models of growing neurites. Neurocomputing. 38-40:31-36. 
Graham, B., Van Ooyen, A. (2004) Transport limited Effects in a model of dendritic branching. Journal of Theoretical Biology 230: 421-432

Groc, L., Petanjek, Z., Gustafsson, B., Ben-Ari, Y., Hanse, E. and Khazipov, R. (2002) In vivo blockade of neural activity alters dendritic development of neonatal CA1 pyramidal cells. European Journal of Neuroscience (16) 1931-1938

Hely, T., Graham, B., and van Ooyen, A. (2001) A computational model of dendritic elongation and branching based on MAP-2 phosphorylation. J. Theor. Biol., 210:375-384.

Hentschel, H.G.E., and Fine, A. (1994) Instabilities in cellular dendritic morphogenesis. Phys. Rev. Lett., 73: 3592-3595.

Hentschel, H.G.E., and Fine, A. (1996) Diffusion-regulated control of cellular dendritic morphogenesis. Proc. R. Soc. B, 263: 1-8.

Hentschel, H.G.E., and van Ooyen, A. (1999) Models of axon guidance and bundling during development. Proc. R. Soc. B, 266: 2231-2238.

Hentschel, H. G. E., and Fine, A. (2003). Early dendritic and axonal morphogenesis. In: A. van Ooyen (ed.), Modelling Neural Development, MIT Press, 2003, Cambridge, MA, pp. 49-74.

Hillman, D.E.(1979). Neuronal shape parameters and substructures as a basis of neuronal form, in: F Schmitt, ed., The neurosciences, 4th study program, MIT Press, Cambridge, MA, pp. 477498. 
Hoffman P.N., and Lasek R.J. (1975) The slow component of axonal transport, identification of major structural polypeptides of the axon and their generality among mammalian neurons. J.Cell Biol., 66: 351-366.

Hua, J.Y. and Smith, S. (2004) Neural activity and the dynamics of central nervous system development. Nature Neuroscience 7:4 327-332

Janulevicius, A., van Pelt, J., and van Ooyen, A. (2003) The effect of dynamic instability of microtubules on growth cone dynamics, Computational Neuroscience Meeting, Alicante, Spain.

Kater, S. B., Mattson, M. P., Cohan, C., and Connor, J. (1988). Calcium regulation of the neuronal growth cone. Trends in Neurosci. 11: 315-321.

Kiddie, G., McLean, D., van Ooyen, A. and Graham, B. (2005). Biologically plausible models of neurite outgrowth. In van Pelt, J., Kamermans, M., Levelt, C., van Ooyen, A., Ramakers, G., and Roelfsema, P., editors, Development, dynamics and pathology of neuronal networks: from molecules to functional circuits, Progress in Brain Research, 147, pages 67-80. Elsevier.

Kobayashi, N., and Mundel, P. (1997) A role of microtubules during the formation of cell processes in neuronal and non-neuronal cells. Cell tissue Res, 291:163-174.

Koch, C., and Segev, I (1998) Methods of Neuronal Modelling: From Ions to Networks. MIT Press, Cambridge MA 
Koene, R.A., Tijms, B., van Hees, P., Postma, F., de Ridder, A., Ramakers, G.J.A., van Pelt , J., van Ooyen, A. (2009). NETMORPH: A Framework for the Stochastic Generation of Large Scale Neuronal Networks With Realistic Neuron Morphologies, Neuroinformatics, 7(3):195210

Li, G., Qin, C., and Wang, Z. (1992) Neurite branching pattern formation: Modelling and Computer simulation. J. Theor. Biol. 157:463-486.

Li, G., Qin, C., and Wang, Z. (1994) On the mechanisms of growth cone locomotion: Modelling and Computer simulation. J. Theor. Biol. 169:255-362.

Li, G., Qin, C., and Wang, Z. (1995) Computer Model of Growth Cone behaviour and Neuronal Morphogenesis. J. Theor. Biol. 174:381-389.

Lohmann, C., Myhr, K..L., and Wong, R.O.L. (2002) Transmitter-evoked local calcium release stabilizes developing dendrites. Nature, 418:177-181, doi:10.1038/nature00850.

Ma, D., Hines, T., Shea and T. Fischer, I.(2000) Axonal Transport of Microtubule-Associated Protein 1B (MAP1B) in the Sciatic Nerve of Adult Rat: Distinct Transport Rates of Different Isoforms. The Journal of Neuroscience 20(6) 2112-2120

Maccioni, R.B., and Cambiazo, V. (1995) Role of Microtubule-Associated Proteins in the Control of Microtubule Assembly. Physiological Reviews, 75:835-857. 
Magistretti, P.J., Pellerin, L., and Martin, J. (2000) Brain Energy Metabolism.

Psychopharmacology - 4th Generation of Progress, Lippincott Williams and Wilkins.

Mason, C. and Erskine, L. (2000) Growth Cone Form, Behaviour and Interactions In Vivo: Retinal Axon Path finding as a Model. Journal of Neurobiology 44:260-270

McLean, D., van Ooyen, A.., Graham, B. (2004) Continuum model for tubulin-driven neurite elongation. Neurocomputing 58-60 511-516

Miller, K., and Samuals, D. (1997) The axon as a Metabolic Compartment: Protein Degradation, Transport and Maximum Length of an Axon. J Theor Biol. 186:373-379.

Miller, F. and Kaplan, D. (2003) Signalling mechanisms underlying dendrite formation. Current Opinion in Neurobiology 13: 391-398

Nowakowski, R., Hayes, N. and Egger, M. (1992) Competitive Interactions during dendritic growth: a simple stochastic growth algorithm. Brain Research 5:6 152-156

Odde, D. J. (1997) Estimation of the diffusion-limited rate of microtubule assembly, Biophysical Journal, 73, 88-96

Pedigo, S., and Williams, R. (2002) Concentration Dependence of Variability in Growth Rates of Microtubules. Biophys J, p. 1809-1819, Vol. 83, No. 4

Redmond, L. and Ghosh, A. (2001) The role of Notch and Rho GTPase signalling in the control of dendritic development. Curr. Opin. Neurobiol., 11: 111-117. 
Samuels, D. C., Hentschel, H. G. E., and Fine, A. (1996). The origin of neuronal polarization: a model of axon formation. Phil. Trans. R. Soc. B. 351: 1147-1156.

Sayas, C., Avila, J. and Wandosell, F. (2002) Regulation of neuronal cytoskeleton by lysophosphatidic acid: role of GSK-3. Biochimica et Biophysica Acta 1582 144-153

Scott, E. and Luo, L. (2001) How do dendrites take their shape? Nature Neuroscience 4:4 359365.

Shefi, O., Harel, A., Chklovskii, D., Ben-Jacob, E. and Ayali, A. (2004). Biophysical constraints on neuronal branching. Neurocomputing 58-60: 487-495

Smith, D.A., and Simmons, R.M. (2001) Models of Motor-Assisted Transport of Intracellular Particles. Biophys J, p. 45-68, Vol. 80, No. 1

Tamori, Y. (1993) Theory of dendritic morphology. Physical Review 48:4 3124- 3129

Tessier-Lavigne, M. and Goodman, C.S. (1996) The molecular biology of axon guidance. Science, 274:1123-1133.

Uemura, E., Carriquiry, A., Kliemann, W. and Goodwin, J. (1995) Mathematical modelling of dendritic growth in vitro. Brain Research 671: 187-194

van Ooyen, A., Graham, B., and Ramakers, G. (2001) Competition for tubulin between growing neurites during development. Neurocomputing 38-40:73-78. 
van Pelt, J., Dityatev, A. and Uylings, H. (1997) Natural variability in the number of dendritic segments: model-based inferences about branching during neurite outgrowth. J. Comp. Neurol., 387:325-340.

van Pelt, J., Graham, B., and Uylings, H. (2003) Formation of Dendritic Branching Patterns. In van Ooyen, A., (Ed.), Modelling Neuronal Development, chapter 4. MIT Press, Cambridge MA, pp 75-94.

van Pelt, J., and Uylings, H. (1999) Natural Variability in the geometry of Dendritic Branching Patterns. In Poznanski, R.R. (Ed.), Modelling in the Neurosciences: From Ionic Channels to Neural Networks, Harwood Academic, Amsterdam, pp 79-108.

van Veen, M., and van Pelt, J. (1992) A Model for Outgrowth of Branching Neurites. J Theor. Biol. 159:1-23.

van Veen, M.P., and van Pelt, J. (1994) Neuritic growth rate described by modelling microtubule dynamics. Bull. math. Biol. 56: 249-273.

Wessells, N.K., and Nuttall, R.P. (1978) Normal branching, induced branching, and steering of cultured parasympathetic motor neurons. Exp. Cell. Res., 115: 111-122.

Whitford, K., Dijkhuizen, P., Polleux, F., and Ghosh, A. (2002) Molecular control of cortical dendrite development. Ann. Rev. Neurosci., 25: 127-149. 
Wong, W.T., and Wong, R.O.L. (2000) Rapid dendritic movements during synapse formation and rearrangement. Current Opinion in Neurobiology, 10:118-124.

Yu, W., Cook, C., Sauter, C., Kurlyama, R., Kaplan, P. and Baas, P. (2000) Depletion of a Microtubule-Associated Motor Protein Induces the Loss of Dendritic Identity. The Journal of Neuroscience 20:15 5782-5791

Zubler, F. and Douglas, R. (2009) A framework for modeling the growth and development of neurons and networks, Frontiers in Computational Neuroscience, 3:(25) 


\section{Image References and attribution}

[1] Santiago Ramón y Cajal. Capas $1^{\text {a }}$ y 2a 1901. (C) Herederos de Ramón y Cajal http://banquete.org/banquete08/IMG/jpg/caja_zoom.jpg

[2] Vanderblit University medical centre

http://www.mc.vanderbilt.edu/histology/images/histology/nervous_tissue/display/Asoma.jpg

[3] Vladimir Baulin 2005

http://vbaulin.front.ru/research/images/microtubule.gif 